\title{
Método de Ponto Proximal e Separadores
}

\author{
Paulo José da Silva e Silva \\ DISSERTAÇÃO DE MESTRADO APRESENTADA AO \\ INSTITUTO DE MATEMÁTICA E ESTATÍSTICA \\ DA \\ UNIVERSIDADE DE SÃO PAULO \\ Curso: Mestrado em Matemática Aplicada \\ Área de Concentração: Ciência da Computação \\ Orientador: Prof. Dr. Carlos Humes Júnior
}

São Paulo, 1997

O aluno recebeu apoio do CNPq e FAPESP. 


\section{Método de Ponto Proximal e Separadores}

Este exemplar corresponde à redaçào final da dissertação apresentada por Paulo José da Silva e Silva. devidamente corrigida e aprovada pela comissão julgadora.

São Paulo, fevereiro de 1997.

Banca examinadora:

Prof. Dr. Carlos Humes Júnior

INE - USP

Prof. Dr. Alfredo Noel Iusem

IMPA - CNPq

Prof. Dr. José Mário Martinez

IMEC' - I'NIC'AMP 
A tudo aquilo que se pode fazer com prazer 


\section{Resumo}

Esta dissertação é centrada em métodos de ponto proximal (MPP), vistos como algoritmos para programação convexa. A importância destes algoritmos para programaçào matemática está, em parte. associada a sua conexão com métodos de multiplicadores, como Lagrangianos aumentados ou o método exponencial de multiplicadores $[11,4]$.

O MPP é caracterizado pela solução de uma seqüência de problemas auxiliares, cujas funções objetivo são a soma da função objetivo original com um termo de regularização. Os primeiros trabalhos $[\mathbf{1 7}, \mathbf{2 0}]$ usavam um múltiplo positivo do quadrado da norma Euclidiana como função de regularização.

Neste texto, apresentam-se resultados de convergência para algoritmos que usam uma generalização da regularização Euclidiana. Basicamente, mostra-se que o MPP converge usando a composição de funções estritamente convexas com normas, sob hipóteses mínimas de diferenciabilidade do termo estritamente convexo. Isto é feito utilizando as idéias de separadores introduzidas por Eaves e Zangwill [9]. Mostra-se ainda que. quando a norma Euclidiana é empregada, a propriedade de Fejér monotonicidade se mantém.

Além destes resultados, que formam a parte central da dissertação, é feita uma revisão de alguns tópicos de convexidade e subdiferenciabilidade e uma breve excursão ao caso em que a regularização é uma distância de Bregman. 


\begin{abstract}
This work focuses on proximal point methods (PPM), viewed as algorithms for convex programming. The importance of such algorithms to the mathematical programming is related to their connection to multiplier methods such as augmented Lagrangian and the exponential method of multipliers $[\mathbf{1 1}, \mathbf{4}]$.

The PPM framework is characterized by the solution of a sequence of auxiliary problems, whose modified objective function is the sum of the original objective function to a regularization term. The first works $[17,20]$ used a positive multiple of the square of the Euclidean norm as its regularization function.

In this work we present convergence results for methods that use a generalization of the Euclidean regularization. Basically, we show that the PPM converges using the composition of strictly convex functions and norms, under mild differentiability assumptions on the strictly convex term. This is done with the ideas of separators introduced by Eaves and Zangwill [9]. Moreover, we show that, using the Euclidean norm, the Fejér monotonicity property is maintained.

In addition to these results, that form the core of the work, we also review some topics of convexity and sub-differentiability, and we do a brief excursion on the case where the regularization is a Bregman distance.
\end{abstract}




\section{Agradecimentos}

Esta lista nunca será completa...

A meus pais, sem os quais não chegaria aqui.

Ao Carlos, sua orientação, paciência e atenção e sua lição do que realmente é ser um mestre.

À Silvina, que mesmo longe está presente sempre.

Ao Marcelo, que me acompanha desde do colegial, todas as discussões e conversas que já tivemos.

A todos os meus amigos, que sempre estiveram do meu lado para comer pizza. falar da vida ou ir ao cinema. 


\section{Sumário}

Notação vii

Capítulo 1. Introdução 1

Capítulo 2. Revisào de Análise Convexa 4

1. Conjuntos convexos 4

2. Funções convexas 14

3. Derivadas direcionais laterais e subgradientes 21

4. Apêndice 34

Capítulo 3. Resultados Gerais e Separadores 38

1. Definiçoes e Resultados Gerais 38

2. Separadores 42

Capítulo 4. Métodos de Ponto Proximal Baseados em Normas 48

1. Ponto Proximal com Norma 48

2. MPPN e problemas restritos 60

Capítulo 5. Métodos de Ponto Proximal Baseados em Funções de Bregman 62

1. Distâncias de Bregman 62

2. O Método 65

3. Apêndice 71

$\begin{array}{lll}\text { Capítulo 6. Conclusào } & 77\end{array}$

$\begin{array}{ll}\text { Referências Bibliográficas } & 78\end{array}$ 


\section{Notação}

- Seja $x$ um vetor do $\mathbb{R}^{n}$ e $\alpha$ um número real não nulo. Para denotar o vetor

$$
\frac{1}{a} x
$$

usar-se-á o abuso de notação

$$
\frac{x}{\alpha}
$$

- Seja $x$ um vetor do $\mathbb{R}^{n}$.

- $\|x\|_{1}=\left|x_{1}\right|+\cdots+\left|x_{n}\right|$;

$-\|x\|_{2}=\sqrt{x_{1}^{2}+\cdots+x_{n}^{2}}$

- $\|x\|_{\infty}=\max _{i=1, \cdots, n}\left\{\left|x_{i}\right|\right\}$;

- $x_{i}$ é a $i$-ésima coordenada de $x$.

- Se $A$ é um subconjunto do $\mathbb{R}^{n}$ e $x$ é um vetor, então:

- $\bar{A}$ denota o fecho de $A$;

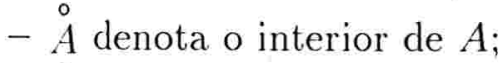

- $\partial A$ denota a fronteira de A.

- $B(x, \alpha)$ denota a bola aberta de centro $x$ e raio $\alpha$, caso não seja especificado a norma utilizada é a Euclidiana.

- $B[x, \alpha]$ denota a bola fechada de centro $x$ e raio $a$, mais uma vez a norma padrão é a Euclidiana.

- Seja $d(\cdot, \cdot)$ uma métrica no $\mathbb{R}^{n}$. A distância de um ponto $x$ ao conjunto $\mathrm{A}$, induzida por esta métrica, é dada por:

$$
d_{P C}(x, A)=\inf _{a \in A}\{d(x, a)\} .
$$

- A função indicadora de $A$, denotada por ind $(\cdot \mid A)$, é definida por

$$
\operatorname{ind}(x \mid A)= \begin{cases}0, & \text { se } x \in A, \\ \infty, & \text { caso contrário. }\end{cases}
$$

- Sejam $A$ e $B$ subconjuntos do $\mathbb{R}^{n}$ : 
- A soma dos conjuntos $A$ e $B$. denotada por $A+B$ é o conjunto

$$
\{c \mid c=a+b, a \in A, b \in B\} .
$$

- O produto um real a por do conjunto $A$, denotado por $\alpha A$ é o conjunto

$$
\{c \mid c=\alpha a, a \in A\} .
$$

Em particular $-A=(-1) A$.

- O conjunto $\{a \mid a \in A, a \notin B\}$ será denotado por $A \sim B$.

- Sejam $a, b$ vetores do $\mathbb{R}^{n}$, então:

- $a<b$ se, e só se, cada componente de $a$ é estritamente menor do que a respectiva componente de $b$.

- $a \leq b$ se, e só se, cada componente de $a$ é menor do que a respectiva componente de $b$ e há pelo menos uma componente onde a desigualdade é estrita.

- $a \leqq b$ se, e só se, cada componente de a é menor do que a respectiva componente de $b$.

- Seja $f(\cdot)$ uma função definida em um abcrto $A$ nos reais. A derivada direcional lateral de $f(\cdot)$ no ponto $x$ na direção $h$, denotada por $f^{\prime}(x, h)$ é o limite

$$
f^{\prime}(x, h)=\lim _{t \rightarrow 0^{+}} \frac{f(x)-f(x+t h)}{t} .
$$

- Seja $V$ um subconjunto do $\mathbb{R}^{n}$, e $f: \mathbb{R}^{n} \mapsto \mathbb{R} \cup\{-\infty, \infty\}$. O conjunto dos argumentos que minimizam $f(\cdot)$ em $V$ é o conjunto de todos os pontos de $V$ onde o mínimo de $f(\cdot)$ em $V$ é atingido, isto é:

$$
\underset{z \in V}{\operatorname{argmin}}\{f(z)\}=\left\{x \mid x \in V e f(x)=\inf _{z \in V}\{f(z)\}\right\} .
$$

Eventualmente este conjunto pode ser vazio. 


\section{CAPÍTLLO 1}

\section{Introdução}

"O Problema de programaçâo não-linear aparece em mithares de formas e pode ser encontrado nas ciências naturais, Física, Engenharia, Economia, Matemática, Finanças e problemas de governo. De fato. mesmo implicaçôes filosóficas já foram analisadas."

Zangwill[24]

Problemas de otimização estào tipicamente associados a operação, ampliação ou projeto de um sistema que deve se comportar de maneira ótima com relação a algum critério. Neste contexto, a programação não-linear ( $\mathrm{PNL}$ ) estuda os casos em que o critério e as restrições de funcionamento do sistema podem ser representadas por um número finito de funções reais de finitas variáveis e o objetivo pode ser visto como único. Por exemplo, suponha que se quer maximizar o lucro de uma fábrica. É comum existirem restrições às decisões (no exemplo poderiam ser disponibilidade de mão de obra, capacidade de produção das máquinas, demanda do mercado, entre outras) que também devem ser tratadas.

O problema clássico de PNL, problema de minimização (PM), é a minimização de uma função escalar $f(\cdot)$ dentro de um conjunto $V$ que é chamado de conjunto de pontos viáveis e que, usualmente, é descrito através de desigualdades envolvendo funçòes.

A invenção e o rápido desenvolvimento do computador digital permitiram a implementação de algoritmos que resolvem casos do $\mathrm{PM}$ que possuam algum tipo de estrutura especial, gerando assim um grande crescimento da programação não-linear na segunda metade deste século. Deste modo, apesar de nova quando comparada a outros ramos da Matemática, ela está estabelecida, com um conjunto de resultados, técnicas sofisticadas e aplicações em diversas áreas como Física, Matemática, Engenharia, Economia e Administração.

Claramente é muito difícil, senão impossível, a solução eficiente do problema geral de PNL. Logo a postura dominante é impor restrições 
à funçào objetivo e ao conjunto viável de modo a permitir o desenvolvimento de métodos de soluçào que explorem a estrutura obtida. Algumas hipóteses usuais sào linearidade, diferenciabilidade. convexidade ou concavidade. condiçòes de qualificaçào como Slater ou reverso-convexa [14], etc.

Um caso particularmente interessante, e muito estudado. é aquele em que a função objetivo, $f(\cdot)$, é convexa e o conjunto viável é um subconjunto não-vazio, fechado e convexo do $\mathbb{R}^{n}$. O método de ponto proximal (MPP) busca resolver este problema substituindo-o por uma seqüência de problemas mais bem comportados, onde a funçào objetivo é estritamente convexa. Isto é possível através da soma de funçòes estritamente convexas à funçào objetivo original. Existem várias classes de funçòes que podem ser usadas, por exemplo o quadrado da norma Euclidiana, distâncias de Bregman ou $\varphi$-divergências $[11,8,5,20,19]$.

O MPP é particularmente interessante na resoluçào de problemas de minimização quando permite a substituição de problemas restritos por uma seqüência de problemas irrestritos. Isto é possivel de duas formas: a primeira, indireta, através de métodos de Lagrangianos aumentados. que muitas vezes podem ser entendidos como aplicação de variações do MPP ao problema dual: a segunda, através da aplicação do MPP com funções de regularização que "absorvem a descrição" do conjunto viável (este é o caso de métodos proximais com distâncias de Bregman). A conexão do MPP com métodos de Lagrangianos foi estudada inicialmente em [19]. Outros bons textos nesta área são [11, 23]. Métodos de ponto proximal com funções de regularização especiais que permitem a eliminação da descrição explícita de conjuntos viáveis simples podem ser encontrados em $[\mathbf{1 1}, \mathbf{2 3}, \mathbf{1 2}, \mathbf{1 3}]$.

A contribuição original desta dissertação é uma generalizaçào do método de ponto proximal que usa como função de regularização o quadrado da norma Euclidiana. Mostra-se que basta considerar composições de funções estritamente convexas e continuamente diferenciáveis com normas quaisquer do $\mathbb{R}^{n}$ (ver capítulo 4). Isto foi possível graças ao uso da ferramenta de separadores apresentada no artigo Generalized Cutting Plane Algorithms de Eaves e Zangwill[9].

O texto se divide da seguinte forma:

- No capítulo 2 é feita uma revisão de análise convexa com destaque para alguns conceitos importantes utilizados nesta dissertação, mas que não estão tipicamente presentes em um primeiro curso de programação não-linear, como, por exemplo, subdiferenciabilidade. Este capítulo tem como objetivo introduzir os conceitos teóricos que serão usados para o tratamento de problemas de minimização convexa. 
- O capítulo 3 pode ser dividido em duas partes bem distintas. Na primeira sào apresentadas as definiçòes básicas e alguns resultados de PNL. Desta maneira, busca-se evitar ambigüidades sobre os problemas que estào sendo tratados. A segunda é uma revisão de alguns resultados do artigo de Eaves e Zangwill que introduz separadores.

- O capítulo 4 apresenta o método geral de ponto proximal e sua regularização original, baseada na norma Euclidiana. Além disto, mostra-se como generalizar esta forma de regularização.

- O capítulo 5 versa sobre métodos de ponto proximal que usam distâncias de Bregman como regularização. Estes métodos são especialmente interessantes por permitirem a substituição de alguns problemas restritos por uma seqüencia de problemas irrestritos. Este capítulo, apesar de não conter contribuiçòes, é apresentado para aumentar a abrangência do texto.

- No capítulo 6 são apresentadas as conclusões do trabalho e sugeridas algumas perspectivas de trabalho futuro. 


\section{CAPíTULO 2}

\section{Revisão de Análise Convexa}

Este capítulo, tem como objetivo apresentar uma revisão de análise convexa, com destaque para resultados e definições que não são comuns durante um curso introdutório de PNL, principalmente subdiferenciabilidade. Porém, assume-se que o leitor possui alguma familiaridade com convexidade (o equivalente aos resultados do Mangasarian[14]). Deste modo, conceitos e resultados básicos serão apresentados sem as demonstraçòes. Se o leitor já estiver familiarizado com análise convexa, a leitura deste capítulo não é necessária para a compreensão do restante do texto.

\section{Conjuntos convexos}

DEFINIÇÃo 1. Um subconjunto $C$ do $\mathbb{R}^{n}$ é dito convexo se, para qualquer $\lambda \in[0,1]$ e quaisquer pontos $x, y \in C$, o ponto definido por $(1-\lambda) x+\lambda y$ pertence a $C$. Geometricamente, isto equivale a afirmar que todo segmento de reta com extremos em $C$ está contido em $C$.

São exemplos de conjuntos convexos:

1. $\emptyset$

2. Um subespaço vetorial (em particular o $\mathbb{R}^{n}$ );

3. Um conjunto composto de um único ponto;

4. Uma reta, uma semi-reta, um segmento de reta, com extremos abertos ou não, semi-espaços e poliedros;

5. Uma bola aberta ou fechada.

1.1. Algumas propriedades conhecidas. Conjuntos convexos gozam de várias propriedades interessantes. Como:

Propriedade 1. A intersecção de conjuntos convexos é um conjunto convexo.

Antes de apresentar as próximas propriedades é necessário definir um pouco de notação.

DEFInIÇÃo 2. Se $A$ e $B$ são subconjuntos do $\mathbb{R}^{n}$ então o conjunto soma de $A$ e $B, A+B$, é definido por:

$$
A+B=\{c \mid c=a+b, a \in A, b \in b\} .
$$


DEFINIÇ̃̃o 3. Se fé um subconjunto do $\mathbb{R}^{n}$ e a é um número real. o conjunto produto de $a$ por $A$, aA é dado por:

$$
a A=\{c \mid c=a a, a \in A\} .
$$

Propriedade 2. A soma de conjuntos convexos é um conjunto cońlvexo.

Propriedade 3. O produto de um real por um conjunto convexo é um conjunto convexo.

Propriedade 4 . O produto cartesiano de conjuntos convexos é um conjunto convexo.

Propriedade 5. A imagem, por uma transformação linear, de um conjunto convexo é um conjunto convexo.

Propriedade 6. Se $A$ é um conjunto convexo então o interior de $A$ é convexo.

Propriedade 7 . Se $C$ é um conjunto convexo fechado de interior não vazio entào $C$ coincide com o fecho de seu interior.

As propriedades 1 a 5 são todas de simples demonstração e as provas podem ser encontradas, por exemplo, no livro Programação Linear: Um Primeiro Curso de Humes e Humes [10].

As propriedades 6 e 7 são um pouco mais sofisticadas e podem ser vistas como corolários do teorema apresentado abaixo. Este teorema apresenta uma importante característica topológica de conjuntos convexos e, por ser não trivial, terá sua demonstração apresentada.

Teorema 1. Seja $C$ um conjunto convexo no $\mathbb{R}^{n}$. Seja $x$ um ponto do interior de $C$ e y um ponto do fecho $C$. Entào $(1-\lambda) x+\lambda y$ pertence ao interior de $C$ para todo $\lambda \in[0,1)$.

Prova. A demonstração aqui apresentada está baseada no livro Conver Analysis de Rockafellar [18].

Seja $B$ a bola de raio unitário e centro em 0 . Deve-se demonstrar que o conjunto $(1-\lambda) x+\lambda y+\epsilon B$ está contido em $C$ para algum $\epsilon>0$. Observa-se que

$$
\begin{aligned}
(1-\lambda) x+\lambda y+\epsilon B & \subset(1-\lambda) x+\lambda(C+\epsilon B)+\epsilon B \\
& =(1-\lambda)\left[x+(1+\lambda)(1-\lambda)^{-1} \epsilon B\right]+\lambda C .
\end{aligned}
$$

Por fim, o último conjunto estará contido em $(1-\lambda) C+\lambda C=C$ quando $\epsilon$ for pequeno o suficiente para que $x+(1+\lambda)(1-\lambda)^{-1} \epsilon B$ esteja contido em $C$. Isto é possivel porque $x \in \stackrel{\circ}{C}$. 
Aćm disto. para o estudo de conjuntos convexos. um conceito fundamental é o de combinaçào convexa. que é apresentado abaixo junto a alguns resultados fundamentais a ele relacionados.

DEFINIÇ̃̃o 4 . Im ponto $\bar{x}$ é dito uma combinaçáo concexa dos pontos $\left\{x^{1}, \ldots, x^{m}\right\}$ se existem reais $a^{i} \geqq 0$ tais que

$$
\bar{x}=a^{1} x^{1}+\cdots+a^{m} x^{m} \quad \text { e } \quad \sum_{i=1}^{m} a^{i}=1 .
$$

DefiniçÃo 5. Dado um conjunto $A$ contido no $\mathbb{R}^{n}$, chama-se casco convero de $A$ ao conjunto de todas as combinaçòes convexas de elementos de $A$. Este conjunto será denotado por $[A]$.

Um resultado trivial é que o casco convexo de um número finito de pontos é um compacto.

Os próximos lemas são resultados clássicos sobre casco convexo e podem ser encontrados, por exemplo, em [10].

LEMA 1. Um conjunto $C$ é convexo se, e somente se. qualquer combinação convexa de elementos de $A$ é um elemento de $A$.

Lema 2. O casco convexo de um conjunto A é o menor (no sentido de inclusâo) conjunto convexo que contém $A$.

1.2. Teoremas de separação. Um fato geométrico bastante intuitivo e interessante sobre conjuntos convexos disjuntos é a existência de hiperplanos separadores, isto é, de hiperplanos que dividem o $\mathbb{R}^{n}$ em dois semi-espaços, cada um contendo um dos conjuntos. Este resultado é fundamental para o desenvolvimento de conceitos como o de subdiferenciabilidade e dualidade em programação não-linear.

Esta seção está baseada no livro Nonlinear Programming: Analysis and Methods de Avriel [1].

DEFINIÇÃo 6. Diz-se que um hiperplano $H$ separa dois conjuntos $A$ e $B$ se $A$ estiver contido em um dos semi-espaços fechados definidos por $H$ e $B$ estiver contido no semi-espaço fechado oposto (figura 1.b) ${ }^{1}$.

DEFINIÇÃO 7 . Diz-se que um hiperplano $H$ separa estritamente dois conjuntos $A$ e $B$ se $A$ estiver contido em um dos semi-espaços abertos definidos por $H$ e $B$ estiver contido no semi-espaço aberto oposto. (figura 1.c). 


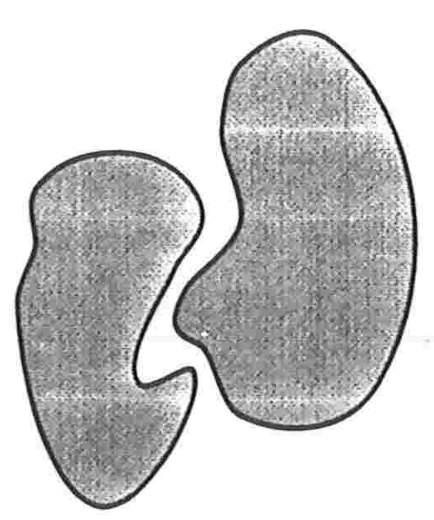

(a)

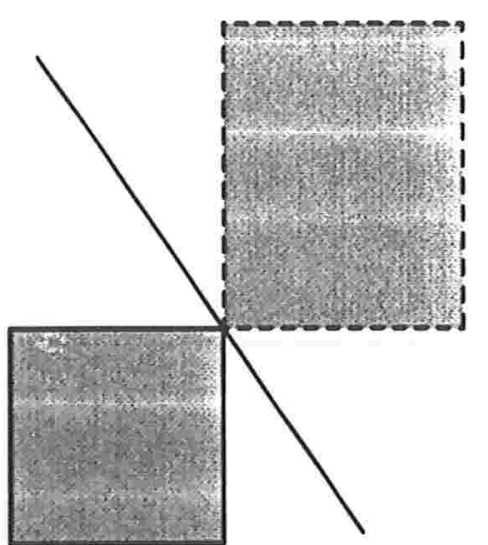

(b)

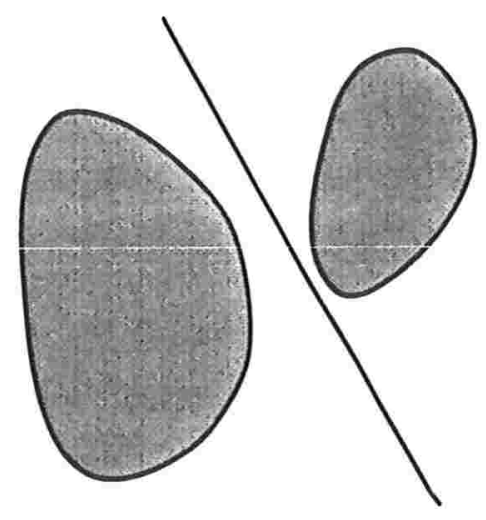

(c)

Figura 1. Possibilidades de separaçào para conjuntos disjuntos: (a) Conjuntos que não podem ser separados por um hiperplano. (b) Conjuntos que podem ser separados por hiperplano, mas não estritamente (o retângulo da direita é aberto e o outro é fechado). (c) Conjuntos que podem ser separados estritamente.

Os principais resultados de separação de convexos sào:

LEMA 3. Seja C um subconjunto do $\mathbb{R}^{n}$ fechado, convero. nào vazio e que não contém a origem. Estão existe um hiperplano que separa estritamente $C$ e a origem.

\footnotetext{
${ }^{1}$ Qualquer plano pode ser descrito na forma do conjunto de pontos que possuem produto escalar constante com um vetor nào nulo $\gamma: H=\left\{x \in \mathbb{R}^{n} \mid\langle\gamma, x\rangle=\beta\right\}$. onde $\beta$ é um real fixado.
} 


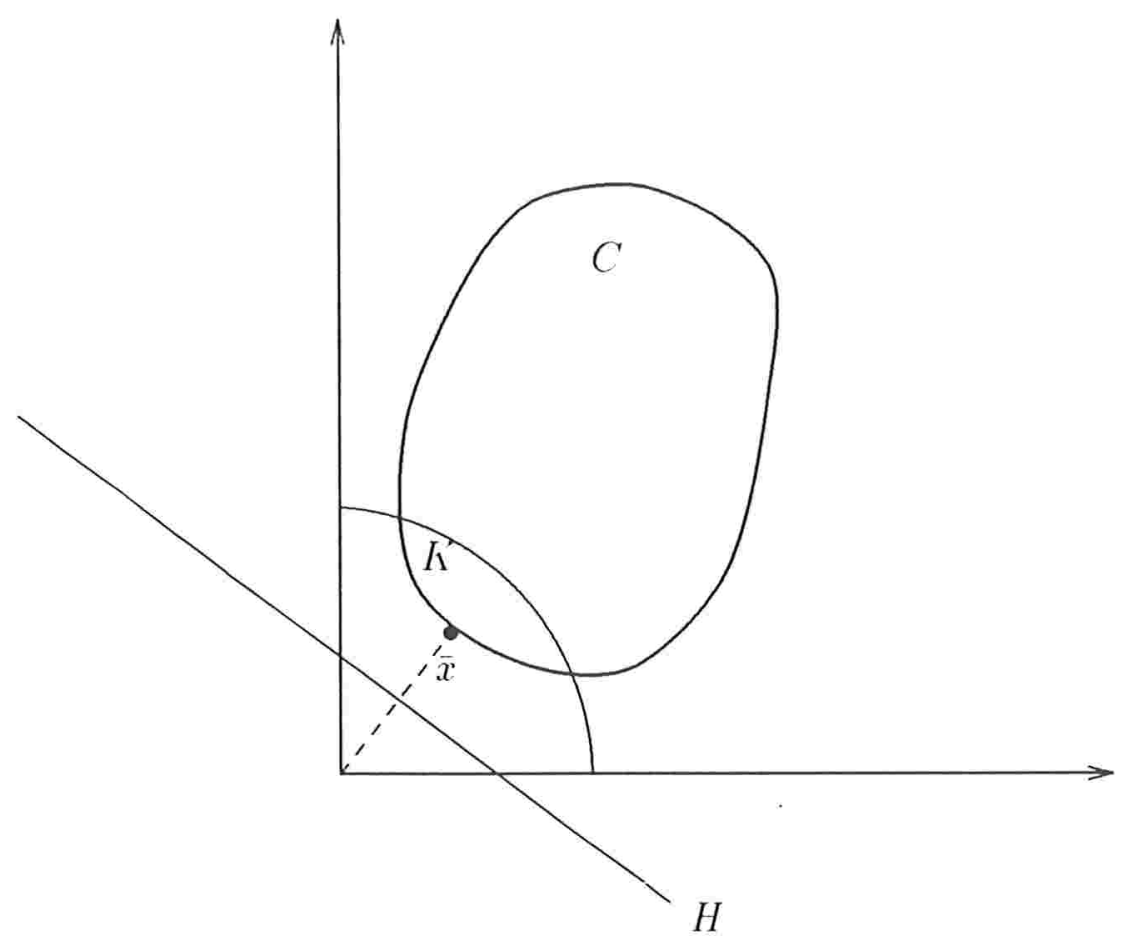

Figura 2. Hiperplano $H$ separando $C$ e a origem.

Prova. Seja $x^{*}$ um ponto de $C$ e $r>\left\|x^{*}\right\|_{2}$. Defini-se o conjunto $K$ como a intersecçào de $B[0, r]$ e $C$. Ele é, claramente, um compacto não vazio. Logo a função contínua $\|\cdot\|_{2}$ admite mínimo em um ponto $\bar{x}$ de $K$.

Como

$$
\begin{aligned}
& \forall x \in K, \quad\|x\|_{2} \geqq\|\bar{x}\|_{2} \\
& \forall x \in C \sim K, \quad\|x\|_{2} \geqq r \geqq\|\bar{x}\|_{2},
\end{aligned}
$$

Segue

$$
\forall x \in C, \quad\|x\|_{2} \geqq\|\bar{x}\|_{2} .
$$

Considera-se agora um ponto $x \in C$. Da expressão anterior e da convexidade de $C$, pode-se concluir que, para qualquer $\lambda \in[0,1]$

$$
\begin{gathered}
\|(1-\lambda) \bar{x}+\lambda x\|_{2}^{2} \geqq\|\bar{x}\|_{2}^{2} \Rightarrow \\
\langle\bar{x}-\lambda \bar{x}+\lambda x, \bar{x}-\lambda \bar{x}+\lambda x\rangle \geqq\langle\bar{x}, \bar{x}\rangle \Rightarrow \\
2 \lambda\langle\bar{x}, x-\bar{x}\rangle+\lambda^{2}\langle x-\bar{x}, x-\bar{x}\rangle \geqq 0 .
\end{gathered}
$$


Supòe-se. por contradiçào. que $\langle\bar{x}, x-\bar{x}\rangle<0$. C'omo na equaçào 1 o termo linear em $\lambda$ domina o termo quadrático. tomando $\lambda$ suficientemente pequeno tem-se uma contradição. Portanto

$$
\begin{aligned}
& \forall x \in C .\langle\bar{x}, x-\bar{x}\rangle \geqq 0 \Rightarrow \\
& \forall x \in C,\langle\bar{x}, x\rangle \geqq\langle\bar{x}, \bar{x}\rangle>0 \quad \text { [Pois } \bar{x} \in C^{\prime} \text { e } 0 \notin C^{\prime} \text { ]. }
\end{aligned}
$$

Tomando $a=\langle\bar{x}, \bar{x}\rangle / 2$, tem-se que

$$
H=\left\{x \in \mathbb{R}^{n} \mid\langle\bar{x}, x\rangle=\alpha\right\}
$$

separa estritamente $C$ e a origem.

Teorema 2. Sejam $C_{1} \in C_{2}$ dois subconjuntos do $\mathbb{R}^{n}$ não vazios, disjuntos, fechados e converos. $C_{2}$ compacto. Entào existe um hiperplano que os separa estritamente.

Prova. Como $C_{2}$ é compacto, o conjunto $C_{1}-C_{2}$ é fechado. ${ }^{2}$ Ainda, como os conjuntos sào disjuntos, $C_{1}-C_{2}$ não contém a origem é convexo.

Usando o lema anterior, tome

$$
H_{C_{1}-C_{2}}=\left\{x \in \mathbb{R}^{n} \mid\langle\gamma, x\rangle=a\right\}
$$

um hiperplano que separa estritamente a origem e $C_{1}-C_{2}$. Sem perda de generalidade, considera-se que $\alpha>0$.

Para quaisquer $u$ e $v$ pertencentes a $C_{1}$ e $C_{2}$. respectivamente, temse

$$
\begin{aligned}
\langle\gamma, u-v\rangle & >a \Rightarrow \\
\langle\gamma, u\rangle & >\alpha+\langle\gamma, v\rangle \Rightarrow \\
\inf _{u \in C_{1}}\{\langle\gamma, u\rangle\} & \geqq \alpha+\langle\gamma, v\rangle \Rightarrow \\
\inf _{u \in C_{1}}\{\langle\gamma, u\rangle\} & \geqq \sup _{v \in C_{2}}\{\langle\gamma, v\rangle\}+a .
\end{aligned}
$$

Como $\alpha>0$, sabe-se que existe $\beta$ tal que

$$
\inf _{u \in C_{1}}\{\langle\gamma, u\rangle\}>\beta>\sup _{v \in C_{2}}\{\langle\gamma, v\rangle\} .
$$

Logo o hiperplano

$$
H=\left\{x \in \mathbb{R}^{n} \mid\langle\gamma, x\rangle=\beta\right\}
$$

separa estritamente $C_{1}$ e $C_{2}$.

\footnotetext{
${ }^{2}$ Note que não basta que os conjuntos sejam fechados. Tome, por exemplo, $C_{1}=\{n+1 / n \mid n \in \mathbb{N}, n \geqq 2\}$ e $C_{2}=\mathbb{N}$. A diferença $C_{1}-C_{2}$ conterá a seqüencia $1 / n$, mas nào conterá o seu limite, o ponto 0 . Para uma demonstração que a soma (ou diferença) de um conjunto compacto com um fechado é fechada, veja o livro Nonlinear Programming de Mangasarian [14], corolário B.3.3.
} 
LEMA 1. Śeja C' um subconjunto do $\mathbb{R}^{n}$ concero. nào vazio. que nào contém a origem. Então existe um hiperplano que separa $C$ e a origem.

Prova. Para cada elemento $x$ de $C$, defina o conjunto

$$
Y(x)=\left\{y \in \mathbb{R}^{n} \mid\|y\|_{2}=1 \mathrm{e}\langle y, x\rangle \geqq 0\right\} .
$$

Este conjunto é compacto e não vazio.

Seja $\left\{x^{1}, \cdots, x^{m}\right\}$ um conjunto finito de pontos de $C$. Como $C$ é convexo, o casco convexo destes pontos é um compacto que nào contém a origem, pois ele está contido em $C$. Pelo lema 3, existe um $\gamma \neq 0$ tal que

$$
\left\langle\gamma, x^{i}\right\rangle>0 \quad \text { Para } i=1, \cdots, m .
$$

Deste modo, tomado o ponto $\gamma /\|\gamma\|_{2}$, segue que

$$
\bigcap_{i=1}^{m} Y\left(x^{i}\right) \neq \emptyset \text {. }
$$

Como os conjuntos $Y(x)$ são compactos, segue, da propriedade de intersecçào finita de compactos ${ }^{3}$, que

$$
\bigcap_{x \in C} Y(x) \neq \emptyset \text {. }
$$

Seja $\gamma$ um elemento qualquer da intersecção anterior, tem-se

$$
\langle\gamma, x\rangle \geqq 0 \text {, para todo } x \in C \text {. }
$$

Logo $H=\left\{x \in \mathbb{R}^{n} \mid\langle\gamma, x\rangle=0\right\}$ é um hiperplano que separa $C$ e a origem.

Teorema 3. Sejam $C_{1}$ e $C_{2}$ dois subconjuntos do $\mathbb{R}^{n}$ não vazios, disjuntos e convexos. Então existe um hiperplano que os separa.

Prova. Pelo lema anterior, tome $\gamma \in \mathbb{R}^{n}$ tal que

$$
H=\left\{x \in \mathbb{R}^{n} \mid\langle\gamma, x\rangle=0\right\}
$$

separa $C_{1}-C_{2}$ e a origem. Conclui-se que para todo $u \in C_{1}$ e todo $v \in C_{2}$ :

$$
\begin{aligned}
\langle\gamma, u-v\rangle & \geqq 0 \Leftrightarrow \quad \text { [Sem perda de gen.] } \\
\langle\gamma, u\rangle & \geqq\langle\gamma, v\rangle \Leftrightarrow \\
\inf _{u \in C_{1}}\{\langle\gamma, u\rangle\} & \geqq \sup _{v \in C_{2}}\{\langle\gamma, v\rangle\} \Rightarrow \\
\exists \beta: \inf _{u \in C_{1}}\{\langle\gamma, u\rangle\} & \geqq \beta \geqq \sup _{v \in C_{2}}\{\langle\gamma, v\rangle\} .
\end{aligned}
$$

\footnotetext{
${ }^{3}$ Ver, por exemplo, Berge [2], capítulo 4, teorema 6 .
} 
Logo

$$
H=\left\{x \in \mathbb{R}^{n} \mid\langle\gamma, x\rangle=3\right\}
$$

é um hiperplano que separa $C_{1}$ e $C_{2}$.

1.3. Ilimitação. Intuitivamente. é natural imaginar que um conjunto convexo ilimitado deve possuir pelo menos uma direçào de ilimitação, como ocorre com poliedros [10]. No caso de poliedros existe uma caracterizaçào simples destas direçôes através do conceito de raios extremais do cone associado ao poliedro. Esta subseção se dedica ao estudo da ilimitação de conjuntos convexos, definindo formalmente alguns conceitos envolvidos e procurando demonstrar alguns resultados. Para um tratamento completo deste tema veja a seção $\delta$ do livro de Rockafellar [18]. Inicialmente, formaliza-se o conceito de direçào de ilimitação.

Para quaisquer conjuntos é possível definir o que é uma direção de ilimitação em um ponto e uma direçào ilimitação. Exemplos podem ser encontrados na figura 3 .

DEFINIÇÃo 8 . Seja $h$ um vetor nào nulo do $\mathbb{R}^{n}$ e $C^{\prime}$ um subconjunto do $\mathbb{R}^{n}$. h é uma direçầo de ilimitação de $C$ em um ponto $\bar{x} \in C$, se, para todo real $\alpha>0$, vale

$$
\bar{x}+\alpha h \in C .
$$

DEFINIÇÃo 9 . Um vetor não nulo do $\mathbb{R}^{n}, h$, é uma direçào de ilimitaçâo de um conjunto $C$, se ela for uma direção de ilimitação em todo ponto de $C$.

Para conjuntos convexos fechados, prova-se a afirmação inicial: todo conjunto ilimitado possui pelo menos uma direçào de ilimitaçào. Para tal, mostra-se que, se o conjunto não for limitado. existe uma direção de ilimitaçào para cada ponto. Em seguida, apresenta-se um resultado bastante interessante que afirma que uma direçào de ilimitaçào em um ponto é uma direçào de ilimitaçào do conjunto.

Lema 5. Sejam $C$ um subconjunto convero e fechado do $\mathbb{R}^{n} \in \bar{x}$ um ponto de $C$. Um vetor $h$ é uma direção de ilimitaçâo de $C$ em $\bar{x}$ se, e somente se, existirem seqüencias $\left(\alpha^{i}\right)_{i \in \mathbb{N}} \in \mathbb{R}_{+} \in\left(h^{i}\right)_{i \in \mathbb{N}} \in \mathbb{R}^{n}$ tais que

$$
\begin{aligned}
\alpha^{i} & \rightarrow \infty, \\
h^{i} & \rightarrow h \neq 0, \\
\bar{x}+\alpha^{i} h^{i} & \in C, \quad \text { para todo } i .
\end{aligned}
$$




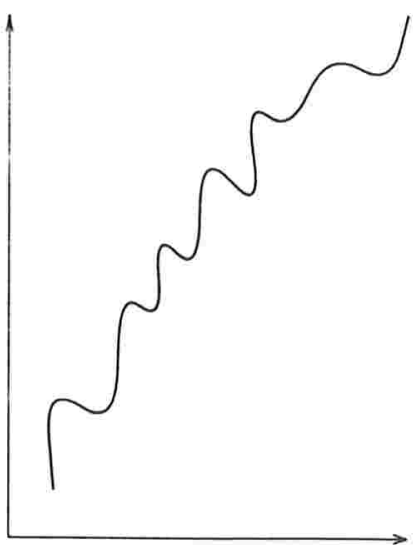

(a)

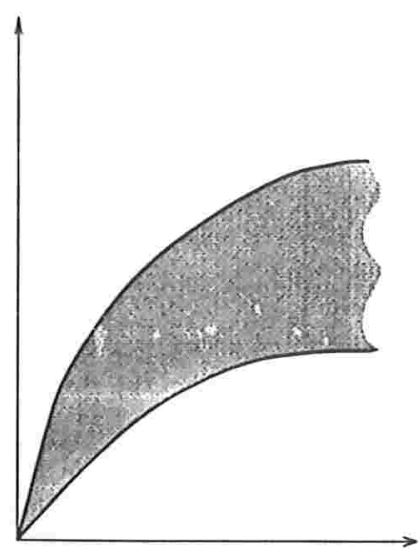

(b)

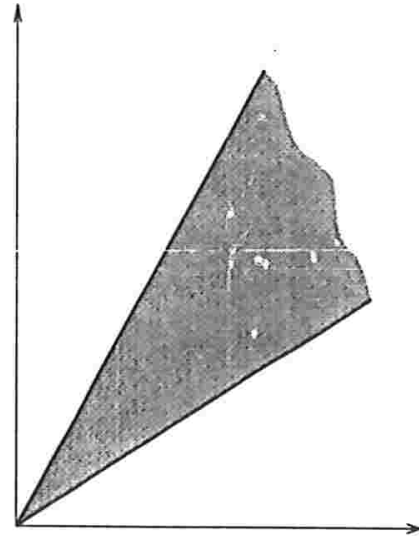

(c)

Figura 3. Conjuntos fechados e ilimitados. Em (a) nào há direçào de ilimitação em nenhum ponto, em (b) existem direções de ilimitação em pontos, mas nào no conjunto e (c) possui as mesmas direções de ilimitaçào em todos os pontos.

Prova. A necessidade é trivial, basta tomar $h^{i}=h$ e $a^{i}=i$. Prova-se a suficiência.

Supondo, por contradição, que a tese não é válida, existe um real $\bar{a}>0$ tal que

$$
\bar{x}+\bar{\alpha} h \notin C .
$$


Entào. pelo teorema 2. de separaçào de convexos. existe um hiperplano separando estritamente $\{\bar{x}+\bar{a} h\}$ e $C^{\prime}$. isto é existe $; \in \mathbb{R}^{\prime \prime}$ e,$j \in \mathbb{R}$ tal que

$$
\left\{\begin{aligned}
\langle\hat{\imath} \cdot \bar{l}+\bar{\alpha} h\rangle & >\beta \\
\langle\bar{\gamma} \cdot \bar{x}\rangle & \left.<\beta . \text { para todo } x \in C^{\prime}\right\}
\end{aligned}\right.
$$

Uma vez que $\bar{x} \in C$. da expressão anterior conclui-se que

$$
\begin{aligned}
\langle\gamma, \bar{x}\rangle+\bar{a}\langle\hat{\gamma}, h\rangle & >\langle\hat{\gamma}, \bar{x}\rangle \\
\bar{\alpha}\langle\gamma, h\rangle & >0 .
\end{aligned}
$$

Por fim. como $\vec{x}+\alpha^{i} h^{i} \in C$ para todo $i$. da equaçào 2 tem-se

$$
\begin{gathered}
\langle\gamma, \bar{x}\rangle+\alpha^{i}\left\langle\gamma, h^{i}\right\rangle\langle\beta<\langle\gamma, \bar{x}\rangle+\bar{\alpha}\langle\gamma, h\rangle \Rightarrow \\
\frac{\alpha^{i}}{\bar{a}} \frac{\left\langle\gamma, h^{i}\right\rangle}{\langle\gamma, h\rangle}<1 .
\end{gathered}
$$

A última equaçào segue de (3).

Como $h^{i}$ converge a $h, \bar{\alpha}>0$ e $a^{i} \rightarrow \infty$, é possivel obter uma contradição tomando o limite para $i \rightarrow \infty$ na expressào acima. Logo $h$ é uma direção de ilimitação de $C$ em $\bar{x}$.

Corclário 1. Se C é um conjunto convero fechado e ilimitado, $C$ possui uma direçào de ilimitaçào em cada um de seus pontos.

Prova. Seja $\bar{x}$ um ponto do conjunto $C$ e $\left(x^{i}\right)_{i \in \mathbb{N}}$ uma seqüência em $C \sim\{\bar{x}\}$ tal que $\left(\left\|x^{i}\right\|_{2}\right)_{i \in \mathbb{N}}$ diverge. Definindo

$$
\begin{aligned}
h^{i} & =\frac{\bar{x}-x^{i}}{\left\|\bar{x}-x^{i}\right\|_{2}} \\
a^{i} & =\left\|\bar{x}-x^{i}\right\|_{2},
\end{aligned}
$$

tem-se que a seqüência $h^{i}$ possui sempre norma 1, logo admite uma subseqüência convergente a um versor. Esta subseqüência convergente de $h^{i}$, junto aos respectivos $\alpha^{i}$ obedecem às condiçòes do lema anterior.

Lema 6. Seja $C$ é um conjunto convero e fechado, um letor $h e ́$ uma direçào de ilimitação de $C$ se, e somente se, hé uma direção ilimitação de $C$ em um ponto $\bar{x}$.

Prova. Mais uma vez a suficiência é trivial. Prova-se a necessidade.

Seja $x$ um ponto qualquer de $C$. Define-se as seqüências:

$$
\begin{aligned}
h^{i} & =\frac{\bar{x}}{i}+h-\frac{x}{i}, \\
a^{i} & =i,
\end{aligned}
$$


Pode-se concluir facilmente que

$$
\begin{aligned}
h^{i} & \rightarrow h . \\
a^{i} & \rightarrow \infty .
\end{aligned}
$$

Como $h$ é direçào de ilimitação em $\bar{x}$ e

$$
\begin{aligned}
x+a^{i} h^{i} & =x+\bar{x}+i h-x \\
& =\bar{x}+i h,
\end{aligned}
$$

segue que $x+\alpha^{i} h^{i} \in C$, para todo $i$. Portanto o lema 5 diz que $h$ é uma direção de ilimitaçào de $C$ em $x$.

Corolário 2. Todo convexo fechado e ilimitado possui uma direçào de ilimitação.

Prova. Aplica-se o corolário 1 e o lema anterior.

\section{Funções convexas}

Uma primeira dificuldade que se encontra ao estudar funçòes convexas é a existência de definições que não são totalmente equivalentes. A definição escolhida nesta dissertação está baseada no livro clássico de Rockafellar, Convex Analysis, com a diferença que aqui se consideram funções que levam a valores em $\mathbb{R} \cup\{\infty\}$, não permitindo o valor $-\infty$. Esta opção pode ser defendida por permitir tratar problemas de minimização restritos como problemas irrestritos ${ }^{4}$, porém sem introduzir as dificuldades técnicas associadas à indeterminações do tipo $\infty+(-\infty)$. Outra razão desta escolha é a ênfase no aspecto geométrico e na relação entre funções e conjuntos convexos.

Como a abordagem escolhida pode levar a cálculos aritméticos envolvendo $+\infty$ ou $-\infty$, apresenta-se uma lista das regras adotadas:

DEFINiÇÃo 10. Regras adotadas para cálculo aritmético envolvendo $+\infty$ e $-\infty$.

$$
\begin{array}{ll}
\alpha+\infty=\infty+\alpha=\infty, & \text { para }-\infty<\alpha \leqq \infty, \\
\alpha-\infty=-\infty+\alpha=-\infty, & \text { para }-\infty \leqq \alpha<\infty, \\
\alpha \infty=\infty \alpha=\infty, \quad \alpha(-\infty)=(-\infty) \alpha=-\infty, & \text { para } 0<a \leqq \infty, \\
\alpha \infty=\infty \alpha=-\infty, \alpha(-\infty)=(-\infty) \alpha=\infty, & \text { para }-\infty \leqq \alpha<0, \\
0 \infty=\infty 0=0=0(-\infty)=(-\infty) 0, & \\
-(-\infty)=\infty, & \\
\inf \emptyset=\infty, \sup \emptyset=-\infty . &
\end{array}
$$

As combinações $\infty+(-\infty)$ e $-\infty+\infty$ não estào definidas e devem ser evitadas.

\footnotetext{
${ }^{4}$ Os conceitos de problema de minimização e a formalização destes comentários se encontra no capítulo 3 .
} 
Definiçõo 11. Seja $f(\cdot)$ uma funçào cujo domínio é um subconjunto $S$ do $\mathbb{R}^{n}$ e com imagens em $\mathbb{R} \cup\{\propto\}$. O conjunto

$$
\{(x \cdot \mu) \in S \times \mathbb{R} \mid \mu \geqq f(x)\}
$$

é chamado epígraft de $f(\cdot)$ e denotado epi $f$. Lima funçào é convera em $S$ se sua epígrafe é um conjunto convexo. Uma funçào é côncava em $S$ se $-f(\cdot)$ é convexa.

DEFinição 12. O domínio efftivo de uma função $f(\cdot)$ definida em um conjunto $S$ é a projeção da epígrafe de $f(\cdot)$ no $\mathbb{R}^{n}$. Ele será denotado por epif.

$$
\operatorname{dom} f=\{x \mid \exists \mu,\{x, \mu) \in \text { epi } f\}=\{x \mid f(x)<\infty\} .
$$

Se $f(\cdot)$ é convexa, este conjunto é convexo, por ser a imagem de um conjunto convexo por uma projeção (uma transformaçào linear).

Outra observação importante é que a convexidade de $f(\cdot)$ é equivalente à convexidade de $f(\cdot)$ restrita a $\operatorname{dom} f$. Vê-se ainda que qualquer função convexa porle ser estendida a todo o $\mathbb{R}^{n}$, definindo $f(x)=\infty$ para todo ponto nào pertencente a $S$. Logo, pode-se considerar que qualquer função convexa está definida em todo $\mathbb{R}^{n}$. Esta abordagem facilita algumas passagens técnicas.

Alguns exemplos de funções convexas são:

1. Funções lineares ou afins (que são os únicos exemplos de funções ao mesmo tempo convexas e côncavas).

2. Uma norma qualquer.

3. $f: \mathbb{R} \mapsto \mathbb{R} \cup\{\infty\}$

$$
f(x)= \begin{cases}\infty, & \text { se } x<-1, \\ 0, & \text { se } x \in[-1,1] \\ \infty, & \text { se } x>1\end{cases}
$$

Existem várias funçòes convexas que estào associadas à conjuntos convexos. Um dos exemplos mais simples e mais úteis é a função indicadora.

DEFINIÇÃo 13. Daṕdo um subconjunto $C$ do $\mathbb{R}^{n}$. A funçào indicadora de $C$ é definida por:

$$
\operatorname{ind}(x \mid C)= \begin{cases}0, & \text { se } x \in C \\ \infty, & \text { caso contrário. }\end{cases}
$$

Observa-se que há uma relaçào um para um entre conjuntos e suas funçòes indicadoras e também que ind $\left(\cdot \mid C^{\prime}\right)$ é convexa se. e somente se, $C$ é um conjunto convexo. 
L'ma hipótese de regularidade que será assumida muitas vezes é que a funçào convexa nào possui domínio efetivo vazio. Isto é:

DEFINis̃o 14. Lna funçào convexa é própria se for finita em pelo menos um ponto de seu domínio.

O próximo resultado liga a definição de funçào convexa apresentada coin a definição mais usual que se encontra, com pequenas variações. em livros como Mangasarian [14] ou Bertsekas [4].

Teorema 4. Seja $f(\cdot)$ uma funçào definida em um conjunto convexo $C$ com imagens em $\mathbb{R} \cup\{\infty\}$. Então $f(\cdot)$ é convexa em $C$ se, $\epsilon$ somente se,

$$
f((1-\lambda) x+\lambda y) \leqq(1-\lambda) f(x)+\lambda f(y), \quad \lambda \in[0,1],
$$

para todo $x$ e y $\mathrm{em} C$.

Prova. Existem dois casos que devem ser considerados.

- $x$ ou $y$ não pertencem a $\operatorname{dom} f$. Sem perda de generalidade supõese que $x \notin \operatorname{dom} f$. Neste caso nada se pode afirmar sobre a convexidade da epígrafe, pois ela não conterá nenhum ponto da forma $(x, \mu)$.

A desigualdade

$$
f((1-\lambda) x+\lambda y) \leqq(1-\lambda) f(x)+\lambda f(y), \quad \lambda \in[0,1],
$$

é sempre válida pois ou o lado direito é $\infty$ ou, $\lambda=1$, e vale a igualdade.

- $x$ e $y$ pertencem a $\operatorname{dom} f$.

- Supòe-se que a desigualdade do enunciado é válida. Dados dois pontos pertencentes a epi $f,(x, \mu)$ e $(y, \nu)$ e $\lambda \in[0,1]$. pode-se concluir que

$$
\begin{aligned}
(1-\lambda) \mu+\lambda \nu & \geqq \lambda f(x)+(1-\lambda) f(y) \\
& \geqq f((1-\lambda) x+\lambda y) .
\end{aligned}
$$

Logo $(1-\lambda)(x, \mu)+\lambda(y, \nu) \in$ epi $f$.

- Supondo que epi $f$ é convexa. Como $(x, f(x))$ e $(y, f(y))$ pertencem a epígrafe, segue que qualquer combinaçào convexa destes dois pontos pertence a epi $f$, isto é:

$f((1-\lambda) x+\lambda y) \leqq(1-\lambda) f(x)+\lambda f(y) . \quad \lambda \in[0,1]$. 
Coroláno 3. (Desigualdade de Jensen) Sejaf(t) definida em um conetro ('com imagens alm $\mathbb{R} \cup\{x\}$. Entäo $f(\cdot)$ í concexa se. t somente st.

$$
f\left(\lambda^{1} x^{1}+\cdots+\lambda^{m} x^{m}\right) \leqq \lambda^{1} f\left(x^{1}\right)+\cdots+\lambda^{m} f\left(x^{m}\right) .
$$

sempre que $\lambda^{1} \geqq 0 \ldots, \lambda^{m} \geqq 0 \epsilon \lambda^{1}+\cdots+\lambda^{m}=1$.

Prova. Uma aplicação direta do teorema anterior e do lema 1.

Um outro conceito, é o de função estritamente convexa

DEFINIÇÃo 15. Uma funçào $f(\cdot)$ de $S$ em $\mathbb{R} \cup\{\infty\}$ é tstritamente convera em $\mathbf{S}$ se $S$ é convexo e

$$
f((1-\lambda) x+\lambda y)<(1-\lambda) f(x)+\lambda f(y), \quad \lambda \in(0.1) .
$$

para todo $x$ e $y$ distintos em $\operatorname{dom} f$.

Funções convexas possuem várias propriedades úteis para sua manipulação. Segue abaixo uma pequena lista de resultados que podcm scr encontrados em qualquer livro de programaçào nào-linear, como [14].

Propriedade 8. A soma de duas funções convexas é convexa.

Propriedade 9. O produto de uma funçào convexa por um escalar positivo é uma função convexa.

Propriedade 10. A composição de uma função convexa com uma função linear é uma função convexa.

Teorema 5. Seja $C$ um convexo $\in f: C \mapsto \mathbb{R} \cup\{\infty\}$ uma funçào nâo constante e convexa $\mathrm{em} C$. Entào nenhum ponto interior de $C$ é ponto de máximo desta funçào.

Prova. Ver Mangasarian [14] teorema 5.2.3.

LEMA 7 . Os conjuntos de nível de uma funçào convexa $f(\cdot)$

$$
C^{\prime}(k)=\left\{x \in \mathbb{R}^{n} \mid f(x) \leqq k\right\},
$$

são convexos para todo real $k^{5}$

Prova. Basta observar que um conjunto de nível é a projeçào da interseç̧ào da epígrafe de $f(\cdot)$ com o semi-espaço $\{(x, \mu) \mid \mu \leqq k\}$.

\footnotetext{
${ }^{5}$ Esta propriedade é muito importante em otimizaçào (quasiconvexidade) e está diretamente ligada a funçòes para as quais todo mínimo local é global.
} 
2.1. Caracterização alternativa de funções convexas diferenciáveis. (Outras propriedades, que surgem quando há diferenciabilidade, permitem a obtenção de definiçòes alternativas de funçòes convexas. Os próximos teoremas sào clássicos e podem ser encontrados em vários livros de programaçào nào-linear (como [14]) ou análise convexa.

Teorema 6. Seje $f(\cdot)$ uma funçâo real. diferenciável. definida em um convero aberto $A$ do $\mathbb{R}^{n}$. Entào $f(\cdot)$ é convera $\mathrm{em} A \mathrm{se}$, $e$ somente se, para quaisquer pontos $x$ e $y$ de $A$ vale

$$
f(x) \geqq f(y)+\langle\nabla f(y), x-y\rangle .
$$

Isto é, $f(\cdot)$ é convexa em A se, e somente se, a aproximaçâo linear de $f(\cdot)$ dada pelo gradiente está sempre abaixo de $f(\cdot)$.

TeOrema 7. Seja $f(\cdot)$ uma funçâo real, diferenciável, definida em um convexo aberto $A$ do $\mathbb{R}^{n} . f(\cdot)$ é estritamente convexa em $A$ se, $e$ somente se, para quaisquer pontos distintos, $x \in y$. de $A$ vale

$$
f(x)>f(y)+\langle\nabla f(y), x-y\rangle .
$$

TeOrema 8. Seja $f(\cdot)$ uma funçâo real, duas vezєs diferenciável, definida em um convexo aberto $A$ do $\mathbb{R}^{n} . f(\cdot)$ é convexa em $A$ se, $e$ somente se, a hessiana de $f(\cdot)$ é semi-definida positiva em todo $A$.

Teorema 9. Seja $f(\cdot)$ uma função real, duas vezes diferenciável, definida em um convexo aberto $A$ do $\mathbb{R}^{n}$. Se a hessiana de $f(\cdot) \dot{e}$ definida positiva em $A$, então $f(\cdot)$ é estritamente convexa em $A$.

2.2. Continuidade. Funções convexas são muito bem comportadas com relação a continuidade e diferenciabilidade. O restante deste capítulo buscará mostrar alguns resultados importantes que envolvem estes conceitos.

Um primeiro exemplo é a continuidade de uma funçào convexa $f(\cdot)$ no interior de seu domínio efetivo, mostrando que comportamentos "estranhos" estão restritos a fronteira de $\operatorname{dom} f .^{6}$

Teorema 10. Uma função convexa $f: \mathbb{R}^{n} \mapsto \mathbb{R} \cup\{\infty\}$ é contínua em qualquer subconjunto aberto de seu domínio efetivo.

Prova. A prova será omitida e pode ser encontrada em vários livros de programação não-linear ou análise convexa. Veja. por exemplo, o livro de Mangasarian [14].

\footnotetext{
${ }^{6} \mathrm{Na}$ verdade valem resultados mais gerais do que os apresentados, envolvendo o interior relativo de conjuntos convexos. Porém, buscou-se evitar a introdução deste conceito para não estender demais este capítulo.
} 
Neste ponto seria natural questionar o quào mal comportada uma funçào convexa pode ser na fronteira de seu domínio efetivo. Ou ainda. se é possivel gerar. a partir de uma funçào convexa qualquer. uma funçào que tenha propriedades "razoáveis" na fronteira.

Uma resposta à última questào é dada pelo uso da operaçào de fecho de uma função, que corresponde à tomar o fecho de sua epígrafe. Através desta operação é possível eliminar parte das descontinuidades na fronteira do domínio efetivo e gerar uma funçào que coincide com a função original em todos os pontos fora da fronteira do domínio efetivo e que é semi-contínua inferior, como definido abaixo:

DEFINIÇÃo 16 . Uma função $f(\cdot)$ de um subconjunto $S$ do $\mathbb{R}^{n} \mathrm{em}$ $\mathbb{R} \cup\{-\infty . \infty\}$ é semi-contínua inferior (s.c.i.) em um ponto $x$ de $S$ se

$$
f(x) \leqq \lim _{i \rightarrow \infty} f\left(x^{i}\right)
$$

para toda seqüência $\left(x^{i}\right)_{i \in \mathbb{N}}$ em $\mathrm{S}$ que converge a $x$ e que o limite de $\left(f\left(x^{i}\right)\right)_{i \in \mathbb{N}}$ existe em $\mathbb{R} \cup\{-\infty, \infty\}$.

A funçào é dita semi-contínua superior se valer a designaldade inversa.

Um exemplo de função convexa que não é semi-contínua inferior ou superior é dado a seguir. Considerando a funçào convexa $f: \mathbb{R}^{2} \mapsto$ $\mathbb{R} \cup\{-\infty, \infty\}$

$$
f(x)= \begin{cases}0, & \text { se }\|x\|_{\infty}<1, \\ 1, & \text { se }\|x\|_{\infty}=1 \text { e } x \in \mathbb{Q}^{2}, \\ 2, & \text { se }\|x\|_{\infty}=1 \text { e } x \in \mathbb{R}^{2} \sim \mathbb{Q}^{2}, \\ \infty, & \text { se }\|x\|_{\infty}>1 .\end{cases}
$$

Observa-se que redefinindo os valores de $f(\cdot)$ para 0 no circunferência de raio 1, pode-se eliminar as descontinuidades. Esta operaçào será formalizada nos próximos dois resultados.

LEMa 8. Seja $F$ um subconjunto convexo do $\mathbb{R}^{n+1}$, então a funçâo $f: \mathbb{R}^{n} \mapsto \mathbb{R} \cup\{-\infty, \infty\}$ definida por

$$
f(x)=\inf \{\mu \mid(x, \mu) \in F\}
$$

é uma função convexa no $\mathbb{R}^{n}$, se, em todo ponto $x$, o ínfimo for maior $q u e-\infty$.

Prova. A prova é conseqüência direta da definição de funçào convexa, uma vez que a epígrafe da função $f(\cdot)$ é. por construção, o conjunto convexo $F+\left\{(0, \mu) \in \mathbb{R}^{n} \times \mathbb{R} \mid \mu \geqq 0\right\}$. 
TeOrema 11. Seja $f(\cdot)$ uma funçào arbitrária do $\mathbb{R}^{n}$ no $\mathbb{R} \cup\{\infty\}$. As condigoes abaixo säo equivalentes:

1. $f(\cdot)$ é semi-contínua inferior no $\mathbb{R}^{n}$.

2. $\{x \mid f(x) \leqq \alpha\}$ é fechado para todo $\alpha \in \mathbb{R}$.

3. A epigrafe de $f(\cdot)$ é um conjunto fechado do $\mathbb{R}^{n+1}$.

Prova. A prova será feita em três partes.

- $1 \Rightarrow 3$ Tome $\left(x^{i} \cdot \mu^{i}\right)_{i \in \mathbf{N}}$ uma seqüência na epígrafe de $f(\cdot)$ convergente a um ponto $(\bar{x}, \bar{\mu})$.

$$
\begin{aligned}
f\left(x^{i}\right) & \leqq \mu^{i}, \forall i \Rightarrow \\
f(\bar{x}) & \leqq \limsup _{i \rightarrow \infty} f\left(x^{i}\right) \leqq \bar{\mu} \Rightarrow \quad[f(\cdot) \text { é semi-cont. inf. }] \\
(\bar{x}, \bar{\mu}) & \in \operatorname{epi} f
\end{aligned}
$$

Logo a epígrafe é fechada.

- $3 \Rightarrow 2$ Seja $\alpha \in \mathbb{R}$ e $\left(x^{i}\right)_{i \in \mathbb{N}}$ uma seqüência pertencente a $\{x \mid$ $f(x) \leqq \alpha\}$ que converge para $\bar{x}$. Então $\left(x^{i}, \alpha\right) \in$ epi $f$ para todo $i$, e, como a epígrafe é fechada, tem-se que $(\bar{x}, \alpha) \in$ epi $f$ e assim

$$
f(\bar{x}) \leqq \alpha \Rightarrow \bar{x} \in\{x \mid \hat{l}(x) \leqq \alpha\} .
$$

- $2 \Rightarrow 1$. Toma-se seqüências $x^{i} \rightarrow \bar{x}$ e $f\left(x^{i}\right) \rightarrow \bar{f}$. Para todo $\alpha>\bar{f}$ tem-se que $f\left(x^{i}\right) \leqq \alpha$ a partir de um certo $i$. Logo para todo $\alpha>\bar{f}$

$$
\begin{aligned}
& x \in \overline{\{x \mid f(x) \leqq \alpha\}}=\{x \mid f(x) \leqq \alpha\} \Rightarrow \\
& x \in \bigcap_{\alpha>\bar{f}}\{x \mid f(x) \leqq \alpha\} \\
& \quad=\{x \mid f(x) \leqq \bar{f}\} .
\end{aligned}
$$

Completanto a prova.

DEFINIÇÃo 17. Dada uma função $f: \mathbb{R}^{n} \mapsto \mathbb{R} \cup\{\infty\}$, chama-se de fecho de $f(\cdot)$, denotado por $\mathrm{cl} f(\cdot)$, à funçào cuja epígrafe é o fecho da epígrafe de $f(\cdot)$.

Lema 9. Seja $f(\cdot)$ uma função convexa definida no $\mathbb{R}^{n}$ com imagens em $\mathbb{R} \cup\{\infty\}$ e $\bar{x}$ um ponto do domínio efetivo onde $f(\cdot)$ é contínua em relaçâo a $\operatorname{dom} f$. Então

$$
f(\bar{x})=\operatorname{cl} f(\bar{x}) .
$$


Prova. Seja $(\bar{x} \cdot \bar{\mu})$ um ponto do fecho da epígrafe de $f(\cdot)$ e $\left(x^{i} \cdot \mu^{i}\right)$ uma seqüência na epígrafe de $f(\cdot)$ convergente a $(\hat{x}, \bar{\mu})$. Segue que:

$$
f(\bar{x})=\lim _{i \rightarrow \infty} f\left(x^{i}\right) \leqq \lim _{i \rightarrow \infty} \mu^{i}=\bar{\mu} .
$$

De onde se conclui que

$$
f(\bar{x}) \leqq \operatorname{cl} f(\bar{x})=\inf \{\mu \mid(\bar{x}, \mu) \in \overline{\operatorname{epi} f}\} .
$$

Por outro lado, como $(\bar{x}, f(\bar{x})) \in$ epi $f$. segue a igualdade.

Corolário 4. Uma funçào convexa coincide com seu fecho no interior de seu domínio efetivo.

Prova. Basta lembrar que a função $f(\cdot)$ é contínua no interior de seu domínio efetivo e aplicar o resultado anterior.

\section{Derivadas direcionais laterais e subgradientes}

Existem vários resultados sobre funções convexas que envolvem suas derivadas direcionais laterais e extensões do conceito de gradiente. Na presença de convexidade, a aproximação linear dada pelo gradiente sempre é menor do que a funçào convexa (ver teorema 6). Ainda mais, em muitos pontos onde uma função convexa não admite diferencial é possível construir funções afins com a propriedade de limitarem a função inferiormente. Os vetores que determinam a inclinação destas aproximaçòes sào os subgradientes ${ }^{\top} \mathrm{e}$, em muitos casos, podem ser usados no lugar do gradiente para o estudo destas funções. Um primeiro passo para a introdução destes conceitos é a investigação das derivadas direcionais laterais de funções convexas. A parte inicial desta seção, até o teorema 14 é baseada no apêndice B do livro de Bertsekas [4].

3.1. Derivadas Direcionais Laterais. Considera-se inicialmente o caso de $f(\cdot)$ ser uma função convexa definida num intervalo real $[a, b]$.

Lema 10. Seja $f:[a, b] \mapsto \mathbb{R}$ uma função convexa. Para quaisquer $x, y, z \in[a, b]$ tal que $x<y<z$ vale

$$
\frac{f(y)-f(x)}{y-x} \leqq \frac{f(z)-f(x)}{z-x} \leqq \frac{f(z)-f(y)}{z-y} .
$$

Prova. Lembrando da definição alternativa de funçào convexa (teorema 4), sabe-se que. como $x<y<z$ :

$$
f(y) \leqq \frac{y-x}{z-x} f(z)+\frac{z-y}{z-x} f(x) .
$$

\footnotetext{
${ }^{7}$ Este conceito será definido com precisão na próxima subseção.
} 
As duas desigualdades seguem de uma reorganizaçào de termos da desigualdade acima. Como ilustraçào far-se-á a prova da primeira desigualdade.

$$
\begin{aligned}
f(y) & \leqq \frac{y-x}{z-x} f(z)+\frac{z-y}{z-x} f(x) \\
(z-x) f(y) & \leqq(y-x) f(z)+(z-x+x-y) f(x) \\
(z-x)(f(y)-f(x)) & \leqq(y-x) f(z)-(y-x) f(x) \\
\frac{f(y)-f(x)}{y-x} & \leqq \frac{f(z)-f(x)}{z-y} .
\end{aligned}
$$

Usando as desigualdades acima é possível estudar as derivadas laterais da função convexa $f(\cdot)$. Para isto, define-se as funções auxiliares.

DEFINIÇÃo 18. Dado $x \in[a, b)$, para todo $t>0$ tal que $x+t \in$ $[a, b]$, é possivel definir a função

$$
f^{+}(x, t)=\frac{f(x+t)-f(x)}{t} .
$$

De modo análogo, dado $x \in(a, b]$, para todo $t>0$ tal que $x-t \in[a, b]$, define-se

$$
f^{-}(x, t)=\frac{f(x)-f(x-t)}{t}
$$

Claramente $\lim _{t \rightarrow 0^{+}} f^{+}(x, t)$ e $\lim _{t \rightarrow 0^{+}} f^{-}(x, t)$ são, respectivamente, as derivadas à direita e à esquerda de $f(\cdot)$ no ponto $x$.

Uma afirmação que se pode fazer das funções definidas acima é:

Lema 11. A função $f^{+}(x, t)$ é nâo decrescente como função de $t e$ $f^{-}(x, t)$ é nâo crescente.

Prova. Tome $0 \leqq t \leqq t^{\prime}$. Usando a primeira desigualdade do lema 10 com $y=x+t$ e $z=x+t^{\prime}$, tem-se:

$$
\begin{gathered}
\frac{f(x+t)-f(x)}{t} \leqq \frac{f\left(x+t^{\prime}\right)-f(x)}{t^{\prime}} \Leftrightarrow \\
f^{+}(x, t) \leqq f^{+}\left(x, t^{\prime}\right) .
\end{gathered}
$$

A demonstração para $f^{-}(x, \cdot)$ é análoga.

De posse deste simples resultado é possível enunciar um importante corolário. 
Corolário 5. Se $f^{+}(x)$ e $f^{-}(x)$ denotem as derieadas à dircita $\mathrm{e}$ esquerda. respectivamente. de $f(\cdot)$ no ponto $x$. entào vale

$$
\begin{aligned}
& f^{+}(x) \in \mathbb{R} \text { ou } f^{+}(x)=-\infty, \forall x \in[a, b) \\
& f^{-}(x) \in \mathbb{R} \text { ou } f^{-}(x)=\infty, \quad \forall x \in(a, b]
\end{aligned}
$$

Prova. Por definição:

$$
f^{+}(x)=\lim _{t \rightarrow 0^{+}} f^{+}(x, t) .
$$

Como $f^{+}(x, l)$ é não decrescente, sabe-se que ou o limite a direita existe ou diverge para $-\infty$.

A demonstração para $f^{-}(\cdot)$ é análoga.

Por fim estes resultados nos levam ao teorema:

Teorema 12. Seja $f:[a, b] \mapsto \mathbb{R}$ uma função convexa, entào:

1. $f^{-}(x) \leqq f^{+}(x), \forall x \in(a, b)$.

2. Se $x$ pertence ao interior do intervalo entâo as derivadas laterais säo reais.

3. Se $x, z \in(a, b)$ e $x<z$ então $f^{+}(x) \leqq f^{-}(z)$.

4. Se $x$ é um real em $(a, b)$ e dé um valor qualquer que satisfaz $f^{-}(x) \leqq d \leqq f^{+}(x)$ então

$$
f(z) \geqq f(x)+d(z-x), \quad \forall z \in[a, b] .
$$

Prova. Divide-se a prova em partes equivalentes aos resultados que se quer demonstrar.

1. Tome $t>0$ suficientemente pequeno para que $x+t$ e $x-t$ pertença ao intervalo $[a, b]$. Usando mais uma vez o lema $10 \mathrm{com}$ $x=x-t, y=x \mathrm{e} z=x+t$ :

$$
f^{-}(x, t) \leqq f^{+}(x, t)
$$

O limite da expressão acima para $t \rightarrow 0^{+}$é o resultado desejado.

2. Tome $t>0$ suficientemente pequeno para que $x+t$ e $x-t$ pertença ao intervalo $[a, b]$. Pelo lema 11

$$
\begin{aligned}
& f^{+}(x) \leqq f^{+}(x, t)<\infty \\
& f^{-}(x) \geqq f^{-}(x, t)>-\infty
\end{aligned}
$$

Do item anterior conclui-se que:

$$
-\infty<f^{-}(x) \leqq f^{+}(x)<\infty .
$$


3. Lsa-se mais uma vez o lema $10 \operatorname{com} x=x, y=(z+x) / 2 \mathrm{e} z=z$. para concluir que

$$
f^{+}(x,(z-x) / 2) \leqq f^{-}(z,(z-x) / 2)
$$

O resultado, entào, segue do fato de $f^{+}(x) \leqq f^{+}(x,(z-x) / 2) \mathrm{e}$ $f^{-}(z) \geqq f^{-}(z,(z-x) / 2)$.

4. Claramente o resultado é válido para $x=z$. Prova-se este resultado para $x<z$. O caso $x>z$ é similar. Como $f^{+}(x, t)$ é não decrescente em $t$, segue que

$$
\frac{f(z)-f(x)}{z-x} \geqq f^{+}(x . t), \quad \forall t \in(0 . z-x) .
$$

Tomando o limite para $t \rightarrow 0^{+}$

$$
\begin{gathered}
\frac{f(z)-f(x)}{z-x} \geqq f^{+}(x) \geqq d \Rightarrow \\
f(z) \geqq f(x)+d(z-x) .
\end{gathered}
$$

Agora, considera-se o caso onde o domínio de $f(\cdot)$ é um subconjunto do $\mathbb{R}^{n}$. A definição de derivada direcional lateral de $f(\cdot)$ na direção $h$ é:

$$
f^{\prime}(x, h)=\lim _{t \rightarrow 0^{+}} \frac{f(x+t h)-f(x)}{t} .
$$

Se, para um ponto $x$ e uma direção $h$, for definida a funçào convexa

$$
f_{h}(t)=f(x+t h)
$$

pode-se, então. verificar facilmente que

$$
f^{\prime}(x, h)=f_{h}^{+}(0) .
$$

Estes comentários levam ao enunciado do corolário:

Corolário 6. Se $f: C \mapsto \mathbb{R}$ é uma função convexa definida em um conjunto $C$ do $\mathbb{R}^{n}$, ex $x$ é um ponto interior de $C$ então $f(\cdot)$ possui derivada direcional lateral em $x$ para qualquer direçào $h$.

Prova. Trivial a partir da equação 6 e do teorema 12. 
3.2. Subgradientes. Quando as funçoes enrolvidas sào convexas. o conceito de gradiente pode ser estendido mantendo várias de suas propricdades. Se $f(\cdot)$ é convexa diferenciável. sabe-se, pelo teorema 6 . que o hiperplano definido por uma aproximação linear de $f(\cdot)$. dada pelo gradiente, separa o espaço $\mathbb{R}^{n+1}$ em duas partes tal que uma destas contém a epígrafe de $f(\cdot)$ (a superior). Xlesmo em pontos onde a função não é diferenciável, pode ser possível a obtenção de aproximações lineares ${ }^{8}$ que sào sempre menores que $f(\cdot)$ (ver figura 4). Os vetores que definem essas aproximações serão chamados de subgradientes de $f(\cdot)$. Esta subseção é dedicada ao estudo de subgradientes e suas relações com as idéias e propriedades de diferenciabilidade.

DEFınição 19. Seja $f: \mathbb{R}^{n} \mapsto \mathbb{R} \cup\{\infty\}$. Um vetor $\gamma$ do $\mathbb{R}^{n}$ é dito um subgradiente de $f(\cdot)$ em um ponto $\bar{x}$ se:

$$
\forall x \in \mathbb{R}^{n} . \quad f(x) \geqq f(\bar{x})+\langle\gamma, x-\bar{x}\rangle .
$$

isto é, a aproximação linear de $f(\cdot)$ no ponto $\bar{x}$, dada por $\gamma$, minora $f(\cdot)$ (figura 4).

Ao mapa ponto conjunto que associa cada ponto $x \in \mathbb{R}^{n}$ ao conjunto de todos os subgradientes de $f(\cdot)$ em $x$ chama-se subdiferencial de $f(\cdot)$ ou mapa de subgradientes de $f(\cdot)$. O subdiferencial de $f(\cdot)$ em um ponto $\bar{x}$ será denotado por $\partial f(\bar{x})$.

Observa-se que uma função que não é constantemente $\infty$ não admite subgradiente em nenhum ponto fora do domínio efetivo.

Subdiferenciais possuem várias propriedades, entre elas destacamse:

Propriedade 11. O subdiferencial de uma função $f(\cdot)$ em $\bar{x}$ é um conjunto convexo.

Prova. Sejam $\gamma, \zeta \in \partial f(\tilde{x})$ e $\lambda \in[0,1]$. Então

$$
\begin{aligned}
\forall x \in \mathbb{R}^{n}, & \lambda f(x) \geqq \lambda f(\bar{x})+\lambda\langle\gamma, x-\bar{x}\rangle \\
& (1-\lambda) f(x) \geqq(1-\lambda) f(\bar{x})+(1-\lambda)\langle\zeta, x-\bar{x}\rangle
\end{aligned}
$$

Somando-se as expressòes conclui-se que

$$
\begin{gathered}
\forall x \in \mathbb{R}^{n}, \quad f(x) \geqq f(\bar{x})+\left\langle(1-\lambda) \zeta+\lambda_{\gamma}, x-\bar{x}\right\rangle \Leftrightarrow \\
(1-\lambda) \zeta+\lambda \gamma \in \partial f(\bar{x}) .
\end{gathered}
$$

\footnotetext{
${ }^{8} \mathrm{Na}$ verdade. aproximaçòes afins. Porém, por simplicidade, permite-se este abuso de linguagem.
} 


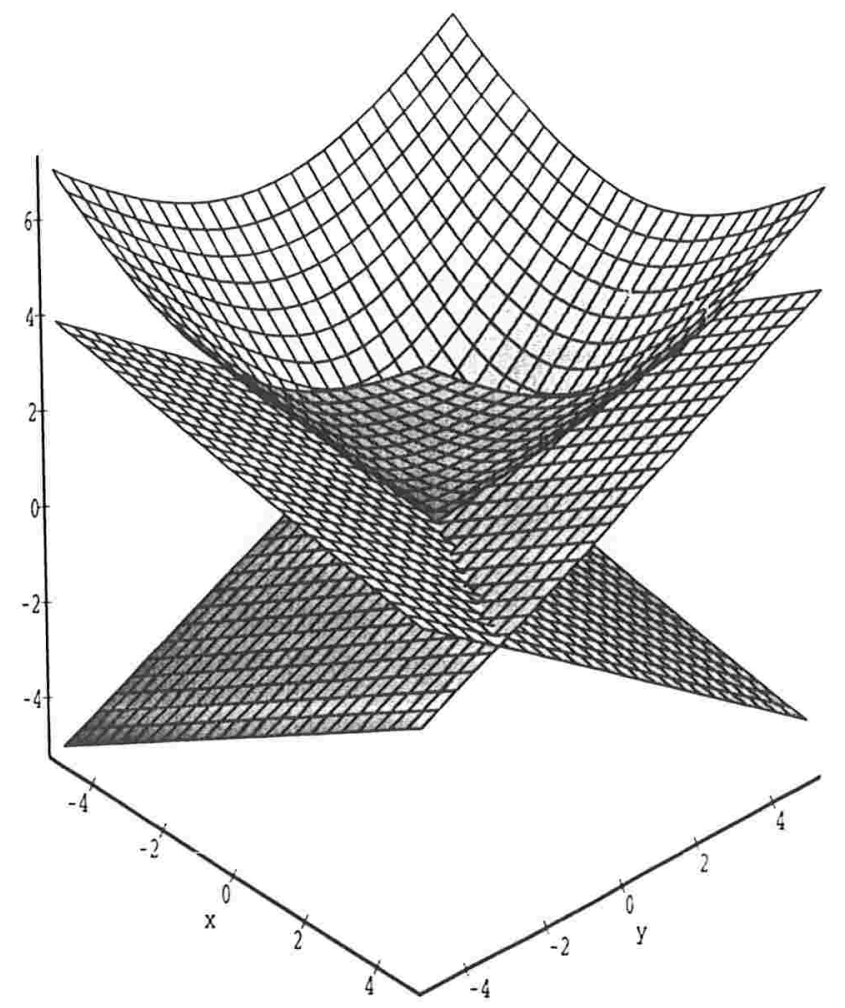

Figura 4. Aproximações lineares "por baixo" da funçào $\|x\|_{2}$ no ponto 0 .

Propriedade 12. Para funções semi-contínuas inferiores o mapa subdiferencial é fechado, isto é: dadas seqüências $\left(x^{i}\right)_{i \in \mathbb{N}}$ e $\left(\gamma^{i}\right)_{i \in \mathbb{N}}$ tais que $x^{i} \rightarrow x^{\infty}, \gamma^{i} \rightarrow \gamma^{\infty}$ e $\gamma^{i} \in \partial f\left(x^{i}\right)$ então $\gamma^{\infty} \in \partial f\left(x^{\infty}\right)$.

Prova.

$$
\begin{aligned}
& \forall x \in \mathbb{R}^{n}, \quad f(x) \geqq f\left(x^{i}\right)+\left\langle\gamma^{i}, x-x^{i}\right\rangle \Rightarrow \\
& \forall x \in \mathbb{R}^{n}, \quad f(x) \geqq \liminf f\left(x^{i}\right)+\left\langle\gamma^{\infty}, x-x^{\infty}\right\rangle \Rightarrow \\
& \forall x \in \mathbb{R}^{n}, \quad f(x) \geqq f\left(x^{\infty}\right)+\left\langle\gamma^{\infty}, x-x^{\infty}\right\rangle .
\end{aligned}
$$

Propriedade 13. Seja $f: \mathbb{R}^{n} \mapsto \mathbb{R} \cup\{\infty\}$ uma função convexa. Se existe um aberto $A$ contendo um ponto $\bar{x}$ tal que

$$
\forall x \in A, \quad f(x) \geqq f(\bar{x})+\langle\gamma, x-\bar{x}\rangle .
$$

então $\gamma$ é um subdiferencial de $f(\cdot)$ em $\bar{x}$, ou seja, a desigualdade acima é válida para todo $x$ do $\mathbb{R}^{n}$. 
Prova. Supòe-se. por contradiçào. que existe $\hat{x} \in \mathbb{R}^{n}$ tal que

$$
f(\hat{x})<f(\bar{x})+\langle\gamma, \hat{x}-\bar{x}\rangle .
$$

Tomando $0<\lambda<1$ pequeno o suficiente para que $(1-\lambda) \bar{r}+\lambda \hat{x}$ esteja em $A$, pode-se afirmar que:

$$
\begin{array}{rlr}
f((1-\lambda) \bar{x}+\lambda \hat{x}) & \leqq(1-\lambda) f(\bar{x})+\lambda f(\hat{x}) & {[f(\cdot) \text { conv.] }} \\
& <(1-\lambda) f(\bar{x})+\lambda(f(\bar{x})+\langle\gamma, \hat{x}-\bar{x}\rangle) & {[\text { Eq. }]} \\
& =f(\bar{x})+\langle\gamma,(1-\lambda) \bar{x}+\lambda \hat{x}-\bar{x}\rangle \Rightarrow & \\
f((1-\lambda) \cdot \bar{x}+\lambda \hat{x}) & <f(\bar{x})+\langle\gamma,(1-\lambda) \bar{x}+\lambda \hat{x}-\bar{x}\rangle . &
\end{array}
$$

Contradizendo a hipótese.

Uma propriedade fundamental de subgradientes é a de monotonicidade, definida abaixo. Esta propriedade está fortemente ligada ao objeto principal de estudo desta dissertação: o método de ponto proximal. Porém, a abordagem escolhida não está baseada na monotonicidade. Para uma discussão mais aprofundada veja o apêndice deste capítulo.

Propriedade 14. O subdiferencial de uma função convexa $f(\cdot)$ é um mapa monótono, isto é, dados pontos $x$ e $y$ do $\mathbb{R}^{n}$ e dois vetores $\gamma \in \partial f(x)$ e $\zeta \in \partial f(y)$ então

$$
\langle\gamma-\zeta, x-y\rangle \geqq 0 .
$$

Prova. Sabe-se que

$$
\begin{aligned}
& f(y) \geqq f(x)+\langle\gamma, y-x\rangle \\
& f(x) \geqq f(y)+\langle\zeta, x-y\rangle
\end{aligned}
$$

Somando-se as duas expressões e simplificando, tem-se

$$
\langle\gamma, x-y\rangle-\langle\zeta, x-y\rangle \geqq 0 \text {. }
$$

Um fato especialmente importante sobre subdiferenciais de funções convexas é a sua ligação com as derivadas direcionais laterais. Na subseção anterior, mostrou-se que funções convexas possuem derivadas direcionais laterais no interior de seu domínio efetivo, qualquer que seja a direção considerada. Sabe-se, do cálculo diferencial, que as derivadas direcionais estão intimamente ligadas a existência de gradientes. Mostrar-se-á a conexão entre as derivadas direcionais laterais e os subgradientes. 
TeOrema 13. Ścja $f: \mathbb{R}^{n} \mapsto \mathbb{R} \cup\{x\}$ uma funçào concera. Para todo ponto $x$ do interior do domínio efetivo de $f(\cdot)$ o subdiferencial é nào cazio c vale a relação

$$
\forall h \in \mathbb{R}^{n}, f^{\prime}(x, h)=\max _{\gamma \in \partial f(x)}\{\langle\gamma, h\rangle\} .
$$

Em particular, se $f(\cdot)$ é diferenciável $\epsilon m x$ entào $\partial f(x)=\{\nabla f(x)\}$.

Prova. Seja $\bar{x}$ um ponto do interior do domínio efetivo de $f(\cdot)$. Inicialmente deve-se destacar que, pelo corolário 6 , as derivadas direcionais laterais em $\bar{x}$ existem e são reais.

Da definição de subgradiente pode-se concluir que:

$$
\begin{aligned}
\gamma \in \partial f(\bar{x}) & \Leftrightarrow f(x) \geqq f(\bar{x})+\langle\gamma, x-\bar{x}\rangle, \quad \forall x \in \mathbb{R}^{n} \\
& \Leftrightarrow f(\bar{x}+\alpha h) \geqq f(\bar{x})+\langle\gamma, \alpha h\rangle, \quad \forall \alpha>0 \text { e } h \in \mathbb{R}^{n} \\
& \Leftrightarrow \frac{f(\bar{x}+\alpha h)-f(\bar{x})}{\alpha} \geqq\langle\gamma, h\rangle, \quad \forall \alpha>0 \text { e } h \in \mathbb{R}^{n} .
\end{aligned}
$$

Por outro lado, lembrando do lema 11, é possível afirmar que

$$
\frac{f(\bar{x}+\alpha h j-f(\bar{x})}{\alpha} \geqq f^{\prime}(\bar{x}, h), \quad \forall \alpha>0 .
$$

Portanto, da definição de derivada direcional lateral e das duas últimas expressões conclui-se que

$$
\gamma \in \partial f(\bar{x}) \Leftrightarrow f^{\prime}(\bar{x}, h) \geqq\langle\gamma, h\rangle, \quad \forall h \in \mathbb{R}^{n} .
$$

Assim $\partial f(\bar{x})$ é a interseção dos semi-espaços fechados

$$
\left\{\gamma \in \mathbb{R}^{n} \mid\langle\gamma, h\rangle \leqq f^{\prime}(\bar{x}, h)\right\}
$$

definidos para todo $h \in \mathbb{R}^{n}$.

Da afirmação acima segue que $\partial f(\bar{x})$ é convexo e fechado. Mais ainda ele é limitado, pois, caso contrário, existiria uma direçào de ilimitação (corolário 2), o que permitiria fazer o lado direito de (\$) tender a infinito.

Prova-se a validade da expressão

$$
\forall h \in \mathbb{R}^{n}, f^{\prime}(\bar{x}, h)=\sup _{\gamma \in \partial f(\bar{x})}\{\langle\gamma, h\rangle\}
$$

De (8), pode-se concluir imediatamente que:

$$
\forall h \in \mathbb{R}^{n}, f^{\prime}(\bar{x}, h) \geqq \sup _{\gamma \in \partial f(\bar{x})}\{\langle\gamma, h\rangle\} .
$$


Seja $\bar{h}$ um vetor qualquer do $\mathbb{R}^{n}$. como $\bar{x}$ é ponto interior do domínio efetivo de $f(\cdot)$, toma-se $B(\bar{x}, r)$ uma bola aberta de centro $\bar{x}$ e raio $r$ contida no domínio efetivo. Definindo os conjuntos convesos

$$
\begin{gathered}
C_{1}=\{(z, \mu) \in B(\bar{x}, r) \times \mathbb{R} \mid f(z)<\mu\} \\
C_{2}=\left\{(z, \mu) \mid z=\bar{x}+a \bar{h}, \mu=f(\bar{x})+a f^{\prime}(\bar{x}, \bar{h}), a>0\right\} .
\end{gathered}
$$

onde $C_{1}$ é convexo por ser o interior da intersecçào da epígrafe de $f(\cdot)$ com o convexo $B(\bar{x}, r) \times \mathbb{R}$, e $C_{2}$ é convexo por ser imagem do intervalo aberto $(0, \infty)$ por uma transformação linear.

É fácil ver que a desigualdade

$$
\forall \alpha>0, \frac{f(\bar{x}+\alpha \bar{h})-f(\bar{x})}{\alpha} \geqq f^{\prime}(\bar{x}, \bar{h})
$$

implica que $C_{1} \cap C_{2}=\emptyset$.

Existe, pelo teorema 3 , um hiperplano que separa $C_{1}$ e $C_{2}$, isto é existe um vetor, não nulo, $(\zeta, \eta) \in \mathbb{R}^{n+1}$ tal que

$$
\begin{aligned}
&\langle\zeta, z\rangle+\eta \mu \leqq\langle\zeta, \bar{x}+\alpha \bar{h}\rangle+\eta\left(f(\bar{x})+\alpha f^{\prime}(\bar{x}, \bar{h})\right), \\
& \forall(z, \mu) \in C_{1}, \forall \alpha>0 .
\end{aligned}
$$

Existem três possibilidades para $\eta$ :

1. $\eta>0$.

Dividindo (10) por $\eta$ e tomando o limite para $\alpha \rightarrow 0^{+}$:

$$
\begin{gathered}
\frac{1}{\eta}\langle\zeta, z\rangle+\mu \leqq \frac{1}{\eta}\langle\zeta, \bar{x}\rangle+f(\bar{x}), \quad \forall(z, \mu) \in C_{1} \Rightarrow \\
\frac{1}{\eta}\langle\zeta, z\rangle+f(z) \leqq \frac{1}{\eta}\langle\zeta, \bar{x}\rangle+f(\bar{x}), \quad \forall z \in B(\bar{x}, r) \quad\left[\mu \rightarrow f(z)^{+}\right] .
\end{gathered}
$$

Portanto a função convexa $1 / \eta\langle\zeta, \cdot\rangle+f(\cdot)$ atinge máximo no ponto $\bar{x}$ do aberto $B(\bar{x}, r)$. De onde se conclui que ela é constante em $B(\bar{x}, r)$ (teorema 5). Assim existe $k \in \mathbb{R}$ tal que

$$
f(x)=k-\frac{1}{\eta}\langle\zeta, x\rangle, \quad \forall x \in B(\bar{x}, r) .
$$

Como "um subgradiente local é global" (propriedade 13), isto implica que

$$
-\frac{\zeta}{\eta} \in \partial f(\bar{x}) \quad \text { e } \quad f^{\prime}(\bar{x}, \bar{h})=\langle\nabla f(\bar{x}), \bar{h}\rangle=\left\langle-\frac{\zeta}{\eta}, \bar{h}\right\rangle .
$$


2. $\eta=0$.

Neste caso a expressão 10 pode ser reescrita como

$$
\begin{gathered}
\langle\bar{\zeta}, z\rangle \leqq\langle\bar{\zeta}, \bar{x}+a \bar{h}\rangle . \quad \forall z \in B(\bar{x}, r) \text { e } a>0 \Rightarrow \\
\langle\bar{\zeta}, z\rangle \leqq\langle\bar{\zeta}, \bar{x}\rangle, \quad \forall z \in B(\bar{x}, r) \quad\left[\text { Lim. para } a \rightarrow 0^{+}\right]
\end{gathered}
$$

Facilmente desta expressào pode-se afirmar que $\zeta=0$. contrariando a definição do hiperplano separador.

3. $\eta<0$.

Dividindo a equação 10 por $\eta$ e tomando o limite para $a \rightarrow 0^{+}$ e, em seguida para $\mu \rightarrow f(z)^{+}$:

$$
\begin{gathered}
\frac{1}{\eta}\langle\zeta, z\rangle+\mu \geqq \frac{1}{\eta}\langle\zeta, \bar{x}+\alpha \bar{h}\rangle+f(\bar{x})+\alpha f^{\prime}(\bar{x}, \bar{h}), \\
\forall(z, \mu) \in C_{1}, \quad \alpha>0 \Rightarrow \\
\frac{1}{\eta}\langle\zeta, z\rangle+\mu \geqq f(\bar{x})+\frac{1}{\eta}\langle\zeta, \bar{x}\rangle, \quad \forall(z, \mu) \in C_{1} \Rightarrow \quad\left[\alpha \rightarrow 0^{+}\right] \\
f(z) \geqq f(\bar{x})+\frac{1}{\eta}\langle\zeta, \bar{x}-\dot{z}\rangle, \quad \forall z \in B(\bar{x}, r) \Rightarrow \quad\left[\mu \rightarrow f(z)^{+}\right] \\
-\frac{\zeta}{\eta} \in \partial f(\bar{x}) \quad[\text { Propriedade 13]. }
\end{gathered}
$$

Tomando $z=\bar{x}, \alpha>0$ em (11) e calculando o limite para $\mu \rightarrow f(\bar{x})^{+}$, tem-se

$$
\begin{gathered}
\frac{1}{\eta}\langle\zeta, \bar{x}\rangle+f(\bar{x}) \geqq \frac{1}{\eta}\langle\zeta, \bar{x}+\alpha \bar{h}\rangle+f(\bar{x})+\alpha f^{\prime}(x, \bar{h}) \Rightarrow \\
\alpha\left\langle-\frac{\zeta}{\eta}, \bar{h}\right\rangle \geqq \alpha f^{\prime}(\bar{x}, \bar{h}) .
\end{gathered}
$$

Portanto, dos três ítens anteriores, pode-se concluir que:

$$
\eta \neq 0, \quad-\frac{\zeta}{\eta} \in \partial f(\bar{x}) \quad \text { e } \quad\left\langle-\frac{\zeta}{\eta}, \bar{h}\right\rangle \geqq f^{\prime}(\bar{x}, \bar{h}) .
$$

Assim, como $\bar{h}$ é um vetor qualquer do $\mathbb{R}^{n}$, a equaçào 9 implica que:

$$
\forall h \in \mathbb{R}^{n}, f^{\prime}(\bar{x}, h)=\sup _{\gamma \in \partial f(\bar{x})}\{\langle\gamma, h\rangle\} .
$$

Como $f^{\prime}(\bar{x}, h)$ é finito para todo $h$, segue que $\partial f(\bar{x})$ é nào vazio e, da compacidade de $\partial f(\bar{x})$, sabe-se que o supremo da expressão acima é atingido.

Por fim, considera-se que $f(\cdot)$ é diferenciável em $\bar{x}$. Qualquer que seja $\gamma \in \partial f(\bar{x})$, da expressão que acaba de ser provada tem-se

$$
\forall h \in \mathbb{R}^{n},\langle\nabla f(\bar{x}), h\rangle=f^{\prime}(\bar{x}, h) \geqq\langle\gamma, h\rangle .
$$


Seja $\epsilon^{i}$ o i-ésimo vetor da base canônica do $\mathbb{R}^{n}$. Substituindo $h$ por $\epsilon^{i}$ $e-\epsilon^{i}$ na expressào anterior. tem-se

$$
\Gamma f(\bar{l})_{i} \geqq \hat{\imath}_{i} \quad \nabla f(\bar{x})_{i} \leqq \gamma_{i} .
$$

De onde se conclui que $\vartheta=\Gamma f(\bar{x})$.

Outro resultado sobre subdiferenciabilidade que é bastante útil:

TeORema 14. Seja $f: \mathbb{R}^{n} \mapsto \mathbb{R}$ uma funçào convexa própria semicontínua inferior $\epsilon .5$ um subconjunto não vazio. fechado $\epsilon$ limitado do interior do domínio efetivo de $f(\cdot)$. Então o conjunto

$$
\partial f(S)=\bigcup\{\partial f(x) \mid x \in S\}
$$

é nào vazio, fcchado c limitado.

Prova. Claramente o conjunto é não vazio, pois os conjuntos $\partial f(x)$ são sempre não vazios no interior do domínio efetivo.

Prova-se que $\partial f(S)$ é fechado. Seja $\left(\gamma^{i}\right)_{i \in \mathbb{N}} \in \partial f(S)$ uma seqüência convergindo a um vetor $\bar{\gamma}$ e $\left(x^{i}\right)_{i \in \mathbb{N}} \in S$, tal que $\gamma^{i} \in \partial f\left(x^{i}\right)$. Como $S$ é compacto, $\left(x^{i}\right)_{i \in \mathbf{N}}$ admite uma subseqüência convergente. Sem perda de generalidade, supòe-se que $\left(x^{i}\right)_{i \in \mathbf{N}}$ converge a um ponto $\bar{x} \in S$ (pois $S$ é fechado). Já que o mapa de subgradientes de uma função convexa semi-contínua inferior é fechado, tem-se que $\bar{\gamma} \in \partial f(\bar{x})$, completando a prova.

Resta provar que $\partial f(S)$ é limitado. Supondo, por contradição que $\partial f(S)$ é ilimitado, então, pelo corolário 2 existe uma direçào de ilimitação em $\partial f(S), h$. Considera-se seqüências $\gamma^{i}=\bar{\gamma}+i h$, onde $\bar{\gamma}$ é um vetor arbitrário de $\partial f(S)$, e $\left(x^{i}\right)_{i \in \mathbf{N}} \in S$, tal que $\gamma^{i} \in \partial f\left(x^{i}\right)$. Como $S$ é compacto, a seqüência $x^{i}$ admite uma subseqüência convergente, $x^{j_{4}}$, a um vetor $\bar{x}$. Do teorema 13 , sabe-se que

$$
f^{\prime}\left(x^{j_{1}}, h\right) \geqq\left\langle\gamma^{j_{1}}, h\right\rangle=j_{i}\|h\|_{2}^{2}+\langle\bar{\gamma}, h\rangle \rightarrow \infty .
$$

Por outro lado, dado $\mu>f^{\prime}(\bar{x}, h)$, existe um $\bar{\alpha}$ tal que

$$
\frac{f(\bar{x}+\alpha h)-f(\bar{x})}{\alpha}<\mu, \quad \forall 0<a<\bar{a} .
$$

Como $x^{j_{i}} \rightarrow \bar{x}$ e $f(\cdot)$ é contínua em $S$, existe um $K \in \mathbb{N}$, tal que, para todo $j_{i}>\Lambda$ vale

$$
\frac{f\left(x^{j_{t}}+\alpha h\right)-f\left(x^{j_{t}}\right)}{a}<\mu, \quad \forall 0<a<\bar{\alpha} .
$$

Dessa forma, pelo lema 11.

$$
f^{\prime}\left(x^{j_{t}}, h\right) \leqq \frac{f\left(x^{j_{t}}+\alpha h\right)-f\left(x^{j_{t}}\right)}{a} .
$$


da expressào anterior segue que:

$$
\limsup _{j_{t} \rightarrow \infty} f^{\prime}\left(x^{j_{t}} \cdot h\right) \leqq \mu
$$

Uma contradição com a expressào 12. Portanto o conjunto $\partial f(S)$ é limitado.

Para que seja razoável a manipulaçào dos subdiferenciais de funções convexas, é interessante obter regras de cálculo de subgradientes que permitam a obtenção do subdiferencial de funções "complicadas" a partir dos subgradientes de funçòes mais simples. Durante o restante desta dissertação encontrar-se-á, em várias oportunidades, funções convexas geradas a partir da soma ou composição de outras funçòes e seria bastante útil possuir teoremas que permitissem a manipulação destes casos de maneira semelhante ao que é feito em cálculo diferencial. Felizmente tais regras existem e serão apresentadas abaixo. Porém a demonstração de alguns dos próximos teoremas envolvem conceitos de sofisticação superior ao escopo desta dissertação. Por isto, apresentar-se-á um caso particular da regra da soma, que será provado, e serão apresentadas referências para os teoremas gerais.

LEMA 12. Sejam $f_{1}(\cdot)$ e $f_{2}(\cdot)$ duas funções convexas definidas $\mathbb{R}^{n}$. Então

$$
\forall x \in \mathbb{R}^{n}, \partial\left(f_{1}+f_{2}\right)(x) \supset \partial f_{1}(x)+\partial f_{2}(x) .
$$

Prova. Seja $\bar{x}$ um ponto do $\mathbb{R}^{n}, \gamma \in \partial f_{1}(\bar{x})$ e $\zeta \in \partial f_{2}(\bar{x})$. Da definição de subgradiente segue:

$$
\begin{aligned}
& \forall x \in \mathbb{R}^{n}, \quad f_{1}(x) \geqq f_{1}(\bar{x})+\langle\gamma, x-\bar{x}\rangle \\
& \forall x \in \mathbb{R}^{n}, \quad f_{2}(x) \geqq f_{2}(\bar{x})+\langle\zeta, x-\bar{x}\rangle
\end{aligned}
$$

Somando as expressões:

$$
\begin{gathered}
\forall x \in \mathbb{R}^{n}, \quad\left(f_{1}+f_{2}\right)(x) \geqq\left(f_{1}+f_{2}\right)(\bar{x})+\langle\gamma+\zeta, x-\bar{x}\rangle \Leftrightarrow \\
\gamma+\zeta \in \partial\left(f_{1}+f_{2}\right)(\bar{x}) .
\end{gathered}
$$

TeOrema 15. Sejam $f_{1}(\cdot)$ e $f_{2}(\cdot)$ duas funções convexas no $\mathbb{R}^{n}$ tais que o domínio efetivo de $f_{1}(\cdot)$ é aberto, $f_{1}(\cdot)$ é diferenciável em seu domínio efetivo $\epsilon$ a interseç̧áo dos domínios efetivos $d \epsilon f_{1}(\cdot) e$ 
$f_{2}(\cdot)$ é nào vazia. Então

$$
\partial\left(f_{1}+f_{2}\right)(x)= \begin{cases}\left\{\nabla f_{1}(x)\right\}+\partial f_{2}(x) . & \text { se } x \in \operatorname{dom} f_{1} \cap \operatorname{dom} f_{2}, \\ \emptyset, & \text { caso contrário. }\end{cases}
$$

Prova. O caso de $x \notin \operatorname{dom} f_{1} \cap \operatorname{dom} f_{2}$ é simples, uma vez que a soma das funçòes vale $+\infty$ neste ponto. Como a função soma tem valor finito em algum ponto, devido a hipótese de $\operatorname{dom} f_{1} \cap \operatorname{dom} f_{2} \neq \emptyset$, ela não poderá ser subdiferenciável em nenhum ponto fora do seu domínio efetivo.

Resta, então, provar o resultado no caso de $x \in \operatorname{dom} f_{1} \cap \operatorname{dom} f_{2}$.

Pelo lema anterior, basta provar que o subdiferencial da soma das funções está contido na soma do subdiferencial de $f_{1}$ com o subdiferencial de $f_{2}$. Isto é, como $f(\cdot)$ é diferenciável, basta provar que se $\gamma \in \partial(f+g)(\ddot{x})$ então $\gamma-\nabla f(\bar{x}) \in \partial g(\bar{x})$. Por hipótese vale:

$$
\begin{array}{ll}
\forall x \in \mathbb{R}^{n}, & f(x)+g(x) \geqq f(\bar{x})+g(\bar{x})+\langle\gamma, x-\bar{x}\rangle \\
\forall x \in \mathbb{R}^{n}, & f(x)=f(\bar{x})+\langle\nabla f(\bar{x}), x-\bar{x}\rangle+o(x-\bar{x})\|x-\bar{x}\|_{2} .
\end{array}
$$

onde $o(z)$ converge para 0 se $z \rightarrow 0$. Substituindo a segunda expressão na primeira:

(13)

$\forall x \in \mathbb{R}^{n}, \quad g(x) \geqq g(\bar{x})+\langle\gamma-\nabla f(\bar{x}), x-\bar{x}\rangle-o(x-\bar{x})\|x-\bar{x}\|_{2}$.

Dado $x \in \mathbb{R}^{n}$, de (13) segue que, para todo $\alpha \in(0,1)$, vale

$$
\begin{aligned}
& g((1-\alpha) \bar{x}+\alpha x) \geqq \\
& g(\bar{x})+\alpha\langle\gamma-\nabla f(\bar{x}), x-\bar{x}\rangle-o(\alpha(x-\bar{x})) \alpha\|x-\bar{x}\|_{2} \Rightarrow \\
& (1-\alpha) g(\bar{x})+\alpha g(x) \geqq \\
& g(\bar{x})+\alpha\langle\gamma-\nabla f(\bar{x}), x-\bar{x}\rangle-o(\alpha(x-\bar{x})) \alpha\|x-\bar{x}\|_{2} \Rightarrow
\end{aligned}
$$

$\alpha g(x)$

$$
\begin{gathered}
\geqq \alpha g(\bar{x})+\alpha\langle\gamma-\nabla f(\bar{x}), x-\bar{x}\rangle-o(\alpha(x-\bar{x})) \alpha\|x-\bar{x}\|_{2} \Rightarrow \\
g(x) \geqq g(\bar{x})+\langle\gamma-\nabla f(\bar{x}), x-\bar{x}\rangle-o(\alpha(x-\bar{x}))\|x-\bar{x}\|_{2} \Rightarrow \\
g(x) \geqq g(\bar{x})+\langle\gamma-\nabla f(\bar{x}), x-\bar{x}\rangle .
\end{gathered}
$$

A última expressão é obtida tomando-se o limite para $a \rightarrow 0^{+}$.

Este último teorema pode ser estendido para: 
TEOREMa 16. (Regra da Soma para subdiferenciais)

Sf jam $f_{1}(\cdot) \ldots f_{m}(\cdot)$ funçóss conétas próprias no $\mathbb{R}^{n}$. e seja $f(\cdot)=f_{1}(\cdot)+\cdots+f_{m}(\cdot)$. Entäo

$$
\forall x \in \mathbb{R}^{n}, \partial f(x) \supset \partial f_{1}(x)+\cdots+\partial f_{m}(x) .
$$

Se os interiores dos domínios efetivos das funçôes $f_{i}(\cdot)$ têm um ponto em comum entào

$$
\forall x \in \mathbb{R}^{n} . \partial f(x)=\partial f_{1}(x)+\cdots+\partial f_{m}(x) .
$$

Prova. Este é um caso particular do teorema 23.8, do livro Convex Analysis de Rockafellar [18].

Para subdiferenciais, além da regra da soma existem diversas variações da regra da cadeia. Um caso que será usado nesta dissertação é:

Teorema 17. (Regra da Cadeia para Subdiferenciais)

Seja $f(\cdot)=g \circ h(\cdot)$, onde $g: \mathbb{R} \mapsto \mathbb{R}$ é continuamente diferenciável e $h: \mathbb{R}^{n} \mapsto \mathbb{R}$ é localmente Lipschitz. Então

$$
\forall x \in \mathbb{R}^{n}, \partial f(x)=g^{\prime}(h(x)) \partial h(x) .
$$

Prova. Este é o corolário 5Q do livro The Theory of Subgradients and its Applications to Problems of Optimization: Convex e Nonconvex Functions também de Rockafellar [22].

\section{Apêndice}

Neste apêndice discutir-se-ão as propriedades de monotonicidade do mapa de subgradientes. Para tal deve-se definir o que é um mapa monótono:

DEFINIÇÃo 20. Um mapa ponto conjunto, $T: \mathbb{R}^{n} \mapsto \mathcal{P}\left(\mathbb{R}^{n}\right)$ é monótono se vale:

$$
\forall x, y \in \mathbb{R}^{n} \text { e } \forall \gamma \in T(x), \zeta \in T(y),\langle\gamma-\zeta, x-y\rangle \geqq 0 .
$$

A propriedade 14 do mapa de subgradientes de uma funçào convexa mostra que ele é monótono.

Outro conceito essencial é o de maximalidade:

DEFINIÇÃo 21. Um mapa $T: \mathbb{R}^{n} \mapsto \mathcal{P}\left(\mathbb{R}^{n}\right)$ é monótono maximal se 
1. $T(\cdot)$ é monótono:

2. Se $T^{\prime}(\cdot)$ é um mapa monótono tal que $T(x)^{\prime} \supset T(x)$ para todo $x$ entào $T^{\prime}=T$.

É possível mostrar que o subdiferencial de uma funçào semi-contínua inferior convexa e própria é monótono maximal. Lma referência para este resultado é o artigo On the maximal monotonicity of subdifferential mappings de Rockafellar [21]. A demonstraçào encontrada neste artigo é baseada no uso de funções convexas conjugadas (fugindo ao escopo desta dissertação). Neste apêndice, apresenta-se-á uma demonstração elementar da maximalidade do subdiferencial de uma funçào convexa com imagens reais. Este resultado é baseado no resultado apresentado por Minty em seu artigo [16], com uma demonstraçào que consideramos mais intuitiva.

Antes de enunciar o teorema, prova-se um lema auxiliar.

Lema 13. Seja $f:[0,1] \mapsto \mathbb{R}$ uma funçào convexa e semi-contínua inferior. Entào nào existem mapas monótonos $T(\cdot), T^{\prime}(\cdot)$ de $[0,1]$ nas partes de $\mathbb{R}$ e um real $\gamma$, tais que, para todo $x \in[0,1]$ :

$$
\begin{aligned}
\partial f(x) & \supseteq T(x) \neq \emptyset, \\
T^{\prime}(x) & = \begin{cases}T(x), & \text { se } x \neq 0 ; \\
T(x) \bigcup\{\gamma\}, & \text { se } x=0, \text { onde } f(1)<f(0)+\gamma .\end{cases}
\end{aligned}
$$

Prova. Seja $\left(x^{i}\right)_{i \in \mathbf{N}}$ uma seqüencia monotonicamente decrescente com $x^{1}=1$, que nunca se anula e converge para 0 .

Seja $\left(\gamma^{i}\right)_{i \in \mathbb{N}}$ uma seqüência qualquer $\operatorname{com} \gamma^{i} \in T\left(x^{i}\right)$ (isso é sempre possivel pois $T(\cdot) \neq \emptyset)$.

Pela monotonicidade de $T^{\prime}(\cdot)$ segue que $\left(\gamma^{i}\right)_{i \in \mathbb{N}}$ é limitada inferiormente por $\gamma$ e monotonicamente não crescente, assim existe $\bar{i}$ tal que:

$$
\gamma^{i} \rightarrow \bar{\gamma} \geqq \gamma \text {. }
$$

Logo

$$
\left\{\begin{array}{l}
\left(x^{i}\right) \rightarrow 0 \\
\gamma^{i} \in \partial f\left(x^{i}\right) \\
\gamma^{i} \rightarrow \bar{\gamma} \geqq \gamma
\end{array}\right\} \Rightarrow \bar{\gamma} \geqq \gamma \quad \text { e } \bar{\gamma} \in \partial f(0) \text { [pois } \partial f(\cdot) \text { é fechado]. }
$$

Portanto

$$
\begin{aligned}
\forall x \in[0,1], & f(x) \geqq f(0)+\bar{\gamma} x \Rightarrow & & {[\bar{\gamma} \in \partial f(0)] } \\
& f(1) \geqq f(0)+\bar{\gamma} \Rightarrow & & \\
& f(1) \geqq f(0)+\gamma & & {[\bar{\gamma} \geqq \gamma] . }
\end{aligned}
$$


Contrariando a forma do mapa $T^{\prime}(\cdot)(f(1)<f(0)+\vartheta)$.

Teorema 18. Seja $f: \mathbb{R}^{n} \mapsto \mathbb{R}$. uma funçào convexa. O mapa subdiferencial de $f(\cdot)(\partial f(\cdot))$ é monótono maximal.

Prova. O fato de $\partial f(\cdot)$ ser monótono foi provado na propriedade 14 , resta provar a maximalidade.

Em primeiro lugar, deve-se destacar que, como $f(\cdot)$ é real em todo $\mathbb{R}^{n}$, ela é uma funçào contínua e possui subdiferencial nào vazio em todo ponto.

Supõe-se, por contradiçào, que $\partial f(\cdot)$ não é maximal. Entào existe um mapa monótono $T^{\prime}(\cdot)$ do $\mathbb{R}^{n}$ nas partes do $\mathbb{R}^{n}$ tal que:

1. $T^{\prime}(x) \supseteq \partial f(x) \neq \emptyset$, para todo $x \in \mathbb{R}^{n}$.

2. $\exists(z, \bar{x}, \bar{\gamma}) \in \mathbb{R}^{n} \times \mathbb{R}^{n} \times \mathbb{R}^{n}$ onde $\bar{\gamma} \in T^{\prime}(\bar{x}) \mathrm{e}$

$$
f(z)<f(\bar{x})+\langle\bar{\gamma}, z-\bar{x}\rangle \text {. }
$$

Definindo a funçào $g:[0,1] \mapsto \mathbb{R}$ por

$$
g(\lambda)=f(\bar{x}+\lambda(z-\bar{x})), \quad \forall \lambda \in[0,1] .
$$

Pode-se concluir que:

(a). Dado $\tilde{\lambda} \in[0,1]$ e $\zeta \in \partial f(\bar{x}+\tilde{\lambda}(z-\bar{x}))$

$$
\begin{gathered}
\forall \lambda \in[0,1], g(\lambda) \geqq g(\tilde{\lambda})+\langle\zeta, \bar{x}+\lambda(z-\bar{x})-\bar{x}-\tilde{\lambda}(z-\bar{x})\rangle \Leftrightarrow \\
\forall \lambda \in[0,1], g(\lambda) \geqq g(\tilde{\lambda})+\langle\zeta,(z-\bar{x})\rangle(\lambda-\bar{\lambda}) \Leftrightarrow \\
\langle\zeta . z-\bar{x}\rangle \in \partial g(\tilde{\lambda})
\end{gathered}
$$

(b). Como

$$
\begin{aligned}
& f(z)<f(\bar{x})+\langle\bar{\gamma}, z-\bar{x}\rangle \Rightarrow \\
& g(1)<g(0)+\langle\bar{\gamma}, z-\bar{x}\rangle .
\end{aligned}
$$

(c). Dado $\tilde{\lambda} \in(0,1]$ e $\zeta \in \partial f(\bar{x}+\tilde{\lambda}(z-\bar{x}))$, pode-se concluir que:

$$
\begin{aligned}
\langle\bar{\gamma}-\zeta, \bar{x}-\bar{x}-\tilde{\lambda}(z-\bar{x})\rangle & \left.\geqq 0 \Leftrightarrow \quad \text { [Pela monot. de } T^{\prime}(\cdot)\right] \\
\langle\zeta-\bar{\gamma}, \bar{\lambda}(z-\bar{x})\rangle & \geqq 0 \Leftrightarrow \\
\bar{\lambda}\langle\zeta, z-\bar{x}\rangle & \geqq \bar{\lambda}\langle\bar{\gamma}, z-\bar{z}\rangle \Leftrightarrow \\
\langle\bar{\zeta}, z-\bar{x}\rangle & \geqq\langle\bar{\gamma}, z-\bar{x}\rangle .
\end{aligned}
$$


Portanto. definindo para $\forall \lambda \in[0.1]$

$$
\begin{aligned}
I(\lambda) & =\underset{\langle\in \mathcal{U}(\bar{x}+\lambda(z-\bar{x}))}{\bigcup}\{\langle\zeta, z-\bar{x}\rangle\} \\
T^{\prime \prime}(\lambda) & = \begin{cases}T(\lambda), & \text { se } \lambda \neq 0: \\
T(\lambda) \cup\{\langle\gamma, z-\bar{x}\rangle\}, & \text { se } \lambda=0 .\end{cases}
\end{aligned}
$$

observa-se. de (a) que $\partial g(\cdot) \supseteq T(\cdot)$. da monotonicidade de $\partial g(\cdot)$ que $T(\cdot)$ é monótono e como $f(\cdot)$ é subdiferenciável em todos os pontos que $T(\cdot) \neq \emptyset$ : de (b) que $g(1)<g(0)+\langle\bar{\gamma}, z-\bar{x}\rangle$ e de (c) que $T^{\prime \prime}(\cdot)$ é monótono, contrariando o lema anterior.

Um fato muito interessante é que o método de ponto proximal pode ser visto como um método de busca de zero de um mapa monótono e maximal. Isto é, se $T(\cdot)$ é um mapa deste tipo, o MPP pode ser usado para encontrar um ponto $\bar{x}$ tal que

$$
0 \in T(\bar{x}) .
$$

Claramente, da definição de subgradientes, quando o mapa considerado é o subdiferencial de uma função $f(\cdot)$, a condição de ser um zero é equivalente a condição do ponto $\bar{x}$ ser um ponto de mínimo da função $f(\cdot)$ no $\mathbb{R}^{n}$. Assim no caso de funçòes semi-contínuas inferiores, convexas e próprias o MPP pode ser usado para buscar o mínimo irrestrito da função. Alguns textos importantes nesta linha são $[15,17,20,11,7]$. 


\section{Resultados; e Separadores}

Este capítulo procura denalmente os problemas que serão tratados durante o restante rtação, bem como apresentar os conceitos e resultados geraisão utilizados. Na primeira seção, recordam-se definições fundała área de programação matemática e também serão enunciad resultados ligando-as ao capítulo sobre análise convexa. Naeguinte apresentar-se-ão algumas definiçôes e resultados rela artigo Generalized cutting plane algorithms[9] sobre funçõesoras e seu uso para a análise de convergência de algoritmos,, 0 a base de nossa abordagem para o estudo de métodos proxim

\section{Definiçêsultados Gerais}

A programação não-linea resolver o problema de minimização de uma função escala subconjunto do $\mathbb{R}^{n}$. Seguindo as definições encontradas em Mian[14]:

DEFInIÇÃo 22. Problemimizaçâo (PM)

Seja $f: X^{0} \mapsto \mathbb{R} \cup\left\{\propto \mathbb{R}^{n}\right.$ e $V$ um subconjunto de $X^{0}$. Encontre, se existir, um pi $V$ tal que o mínimo de $f(\cdot)$ no conjunto $V$ seja atingido en

$$
f(1\{f(x)\},
$$

ou, usando outra notação,

$$
\bar{x} 1\{f(x)\} .
$$

A função $f(\cdot)$ é conhecida câo objetivo e I conjunto de pontos viáveis.

Geralmente $V$ é dado $n$

$$
V=\mid g(x) \leqq 0\}
$$

onde $g: X^{0} \mapsto \mathbb{R}^{m}$ e é chanunçào de restrição ou, simplesmente, restrições. 
DEFINIÇÃo 26. O PM será chamado de problema de programação convera quando $f(\cdot)$ for uma funçào convexa e $I^{*}$ for um conjunto convexo. Em particular, se $I$ for dado através de desigualdades basta que $g(\cdot)$ seja tal que $g_{i}(\cdot)$ é convexa para todo $i=1 \ldots, m$.

Como apresentado no capítulo anterior. funçòes e conjuntos convexos gozam de várias propriedades especiais que se mostrarào interessantes no contexto de programação matemática. Um exemplo disto é o próximo resultado.

Lema 15. Para programação convexa, todo mínimo local é global.

Prova. Assuma que $\bar{x}$ é um mínimo local para alguma bola aberta $B(\bar{x}, \epsilon)$. Seja $x$ um ponto qualquer de $V$. Tem-se que

$$
x_{\lambda}=\lambda x+(1-\lambda) \bar{x} \in V, \text { para todo } \lambda \in[0,1],
$$

pois $V$ é convexo. Tomando $\lambda>0$ pequeno o suficiente para que $x_{\lambda} \in B(\bar{x}, \epsilon)$ temos

$$
\begin{aligned}
f(\bar{x}) & \leqq f(\lambda x+(1-\lambda) \bar{x}) & & {[\text { Pois } \bar{x} \text { solve P.ML }] } \\
& \leqq \lambda f(x)+(1-\lambda) f(\bar{x}) \Rightarrow & & {[f(\cdot) \text { é convexa }] } \\
f(\bar{x}) & \leqq f(x) & & {[\lambda>0] }
\end{aligned}
$$

Ou seja, no caso convexo, os problemas de minimização local e global são equivalentes, o que, naturalmente, não vale para qualquer PM. Em programação não-linear observa-se que a estrutura de convexidade é muito forte, permitindo a obtenção de bons resultados teóricos que geram algoritmos considerados eficientes para a solução do problema.

Outros resultados que usam bem a estrutura de convexidade são:

LEMA 16. Considere um problema de programaçâo convexa com função objetivo estritamente convexa. Se existir uma soluçào do problema, ela é única.

Prova. Supõe-se, por contradição, que existem duas soluções $x_{1}^{*} \mathrm{e}$ $x_{2}^{*}$. Claramente, da convexidade do conjunto viável, $z=0.5 x_{1}^{*}+0.5 x_{2}^{*}$ é viável e

$$
\begin{aligned}
f(z) & <0.5 f\left(x_{1}^{*}\right)+0.5 f\left(x_{2}^{*}\right) & & {[f(\cdot) \text { é estrit. conv. }] } \\
& =f\left(x_{1}^{*}\right) . & & {\left[f\left(x_{1}^{*}\right)=f\left(x_{2}^{*}\right)\right] }
\end{aligned}
$$

Contradição com a otimalidade de $x_{1}^{*}$.

LEma 17. Seja $f(\cdot)$ uma função convexa. O ponto $\bar{x}$ é uma solução do problema de minimização irrestrita de $f(\cdot)$ se, $\epsilon$ somente se,

$$
0 \in \partial f(\bar{x}) \text {. }
$$


Prova. Segue trivialmente. da definiçào de subgradiente.

Corolário T. O conjunto de soluçòes da minimizaçào irrestrita de uma funçào convera semi-contínua inferior é fechado.

Prova. Basta usar o lema acima e o fato do mapa de subdiferenciais, neste caso, ser fechado.

Curolário 8. Se a funçào objetivo $f(\cdot)$ for convexa e o conjunto viável $\mathrm{V}$ for um convexo aberto tal que $V \mathrm{n} \operatorname{dom} f \neq \emptyset$ entào $x$ é uma soluçâo do problema de minimização se, e somente se,

$$
0 \in \partial f(\bar{x}) \quad \in \quad \bar{x} \in V .
$$

Prova. Considere o problema irrestrito equivalente

$$
\min _{x \in \mathbb{R}^{n}}\{f(x)+\operatorname{ind}(x \mid V)\}
$$

Pelo teorema anterior $\bar{x}$ é mínimo do problema irrestrito se, e somente se,

$$
0 \in \partial(f(\cdot)+\operatorname{ind}(\cdot \mid V))(\bar{a}) .
$$

Nas condições do corolário, a função ind( $\cdot \mid V)$ é diferenciável em $V$, com gradiente nulo, logo, vale a regra da soma de subgradientes dada no teorema 15. Deste modo, a expressão anterior é equivalente a:

$$
0 \in \partial f(\bar{x}) \quad \text { e } \quad \bar{x} \in V .
$$

Esta última relação é verdadeira porque ind( $\cdot \mid V)$ não é subdiferenciável fora de $V$ e tem derivada nula neste conjunto, uma vez que $V$ é aberto e ind $(\cdot \mid V)$ é constante em $V$.

Para a solução de problemas de minimizaçào é comum o uso de métodos que geram seqüências que convergem a uma soluçào. Uma propriedade interessante no estudo de convergência é a de Fejér monotonicidade. Ela será usada na análise de métodos de ponto proximal baseados na norma Euclidiana.

DefiniçÃo 27. Dada uma seqüência $\left(x^{i}\right)_{i \in \mathbb{N}}$ e $G \subset \mathbb{R}^{n}$, ela é Fejér monótona em relaçâo a $G$ se, para alguma norma $\|\cdot\|$ do $\mathbb{R}^{n}$ :

$$
\left\|x^{i+1}-g\right\| \leqq\left\|x^{i}-g\right\|, \quad \forall g \in G .
$$

Deve-se destacar que a propriedade acima é mais forte do que a idéia da seqüência não se afastar do conjunto $G$. pois a seqüência não se afasta de cada ponto de $G$. O principal teorema ligado a esta propriedade é: 
Teoreara 19. Seja $G \neq \emptyset$ um subconjunto do $\mathbb{R}^{n} \in\left(x^{i}\right)_{i \in \mathbb{N}}$ uma seqüencia Fejér Monótona em relação a $(i$. Então

1. $\left(x^{i}\right)_{i \in \mathbf{N}}$ é limitada.

2. Se existe subseqüência $\left(x^{j_{t}}\right)_{i \in \mathbf{N}}$ tal que $x^{j_{t}} \rightarrow g \in G$ entrio $x^{i} \rightarrow g$.

Prova. A notação $\|\cdot\|$ será usada para a norma censiderada.

$O$ ítem 1 é trivial, pois toda seqüência estará contida na bola $B\left(g, x^{1}\right)$, para qualquer $g \in G$.

Supondo que existe uma subseqüencia $x^{j_{t}}$ convergente a um ponto $g \in G$, dado $\epsilon>0$, tome $x^{j_{N}}$ um ponto da subseqüência em $B(g, \epsilon)$. Então, da definição de Fejér monotonicidade, segue que

$$
\forall i>j_{N},\left\|x^{i}-g\right\| \leqq\left\|x^{j_{N}}-g\right\|<\epsilon
$$

Provando, então, que $x^{i} \rightarrow g$.

Esses são os principais conceitos gerais que serão utilizados no decorrer da dissertação. A seguir, introduz-se a idéia de separadores que servirá de base para o estudo de métodos de ponto proximal feito no capítulo 4, onde estăo os principais resultados deste trabalho.

\section{Separadores}

Em seu artigo "Generalized Cutting Plane Algorithms"[9], Eaves e Zangwill abordam o problema de minimização através da resolução de uma seqüência de problemas mais simples, cujas soluçôes se aproximam das soluções do PM em algum sentido. Para isto os autores fazem uso de métodos de planos de corte, unificando e estendendo a teoria destes algoritmos.

Com o objetivo de dar um tratamento coeso à teoria dos métodos de planos de corte, é introduzido o conceito de separador:

DEFINIÇÃo 28. Sejam $X$ e $G$ dois subconjuntos fechados em um espaço métrico, tal que $X^{\prime} \supset G$. Uma função $\delta(\cdot)$, de $X \sim G$ nos reais estendidos, é uma função separadora (ou um separador em $X \sim G$ ) se:

1. $\delta(\cdot)$ é não negativa;

2. $\left(x^{i}\right)_{i \in \mathbf{N}} \in X \sim G, x^{i} \rightarrow x^{\infty}$ e $\delta\left(x^{i}\right) \rightarrow 0 \Rightarrow x^{\infty} \in G$.

$\mathrm{O}$ conjunto $G$ será conhecido como conjunto alvo.

Um exemplo óbvio de separador é a distância entre um ponto $x \in$ $X$ e o conjunto $G\left(\delta(x)=d_{P C}(x, G)\right)$, definida da maneira usual a partir da métrica dada. Também é claro que o mínimo de uma coleção finita de separadores é um separador, qualquer múltiplo positivo de um separador é um separador e, se $\delta(\cdot)$ é um separador e $\delta(\cdot) \leqq \delta^{\prime}(\cdot)$ então $\delta^{\prime}(\cdot)$ é um separador. 
Lma definiçào alternativa de separadores é dada no próximo lema.

Lema 18. S' métrico. tal que $X^{\prime} \supset G$. Lima funçiòo $\delta: X \sim G \mapsto \mathbb{R} \cup\{-\infty . \infty\}$ é um separador se, $t$ somente se, tiver limite inferior positivo em cada subconjunto compacto nào vazio de $X^{\prime} \sim G$.

Prova. A prova está dividida em duas partes.

- Seja $I i$ um compacto nào vazio de $X \sim G$. Como $\delta(\cdot) \geqq 0$ Claramente vale

$$
\inf _{x \in K^{\cdot}}\{\delta(x)\} \geqq 0 .
$$

Supondo, por contradição, que vale a igualdade, então existe uma seqüência $\left(x^{i}\right)_{i \in \mathbb{N}} \subset K$ tal que $\delta\left(x^{i}\right) \rightarrow 0$.

Seja $x^{j_{t}}$ uma subseqüência convergente a um ponto $x^{x}$ então. pela definiçào de separador, segue que $x^{\infty} \in G$.

Por outro lado $x^{\infty} \in K$, pois $K$ é fechado, contrariando o fato de $K \subset X \sim G$. Isto prova a suficiência.

- Considera-se que $\delta(\cdot)$ tem limite inferior positivo em todo compacto de $X \sim G$.

Claramente $\delta(x) \geqq 0$ para todo $x \in X \sim G$ pois um conjunto com um único ponto é compacto.

Supondo que existem seqüências $x^{i} \rightarrow x^{\infty}$ e $\delta\left(x^{i}\right) \rightarrow 0$, com $\left(x^{i}\right)_{i \in \mathbf{N}} \subset X \sim G$. Como $X$ é fechado segue que $x^{\infty} \in X$.

Supondo, por contradição, que $x^{\infty} \notin G$, então existe uma bola fechada de centro $x^{\infty}, B$, que não intercepta $G$. Defina $K^{\prime}=$ $B \cap X$. Claramente $K$ é um subconjunto compacto (interseção de um compacto com um fechado) de $X \sim G$ e o fato de $\delta\left(x^{i}\right) \rightarrow 0$ contraria a hipótese de $\delta(\cdot)$ ter limite inferior positivo, concluindo a demonstraçào.

A partir do conceito de separador, é apresentado em [9] um algoritmo abstrato que serve de base para diversos métodos de plano de corte. bem como para a análise de convergência através de separadores.

"Algoritmo Mestre de Planos de Corte" - AMPC Seja $\delta(\cdot)$ um separador em $X \sim G$.

1. Inicializaçào: Tome $x^{1}$ qualquer em X'.

2. Iteraçào: Se $x^{i} \in G$ pare;

Senão calcule $x^{i+1} \in \mathrm{X}$ tal que

$$
x^{i+1} \notin B\left(x^{j}, \delta\left(x^{j}\right)\right), \quad j=1 \ldots . .
$$




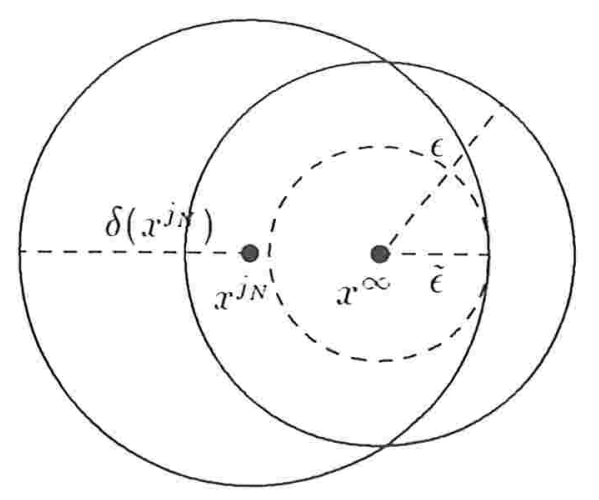

Figura 1. Contradição com a convergência de $\left(x^{j_{l}}\right)_{i \in \mathbf{N}}$.

Observando-se o algoritmo acima, pode-se constatar a importância da escolha de separadores $\delta(\cdot) \leqq d_{P C}(\cdot, G)$. Pois, caso contrário, seria possível "jogar fora" partes do conjunto alvo ao executá-lo. Assim, eventualmente, o algoritmo poderia não ser capaz de gerar o próximo ponto.

Um resultado obtido imediatamente é:

Teorema 20. Sejam $X$ e $G$ dois subconjuntos fechados em um espaço métrico, tal que $X \supset G$ e $\left(x^{i}\right)_{i \in \mathbf{N}}$ uma seqüencia gerada pelo AMPC. Entào todo ponto de acumulaçâo desta seqüência pertence a $G$.

Prova. Considere uma subsequência $\left(x^{j_{i}}\right)_{i \in \mathbf{N}}, x^{j_{i}} \rightarrow x^{\infty}$. Busca-se provar que $\delta\left(x^{j_{1}}\right) \rightarrow 0$.

Para isto supõe-se, por contradição, que o resultado nào é válido. Entào $\exists \epsilon>0$, tal que, para todo $K \in \mathbb{N}$ existe $N>K$ (e conseqüentemente $\left.j_{N}>j_{K}\right)$ onde $\delta\left(x^{j_{N}}\right)>\epsilon$.

Tome a bola aberta $B\left(x^{\infty}, \epsilon\right)$ e um indice $N$ tal que $\delta\left(x^{i_{N}}\right)>\epsilon$ e $x^{j_{N}} \in B\left(x^{\infty}, \epsilon\right)$. Defina $\tilde{\epsilon}=\delta\left(x^{j_{N}}\right)-d\left(x^{j_{N}}, x^{\infty}\right)>0$. Pela definiçào do $\mathrm{AMPC}, \forall j_{i}>j_{N}, x^{j_{i}} \notin B\left(x^{j_{N}}, \delta\left(x^{j_{N}}\right)\right) \supset B\left(x^{\infty}, \widetilde{\epsilon}\right)$ (ver figura 1), contrariando a convergência de $x^{j_{\mathrm{t}}}$. Conclui-se que

$$
\delta\left(x^{j_{i}}\right) \rightarrow 0 \text {. }
$$

$\mathrm{E}$ assim $x^{\infty} \in G$, pela definição de separador.

Tem-se como objetivo utilizar estes resultados na solução de problemas de minimização. Um passo natural é buscar relacionar separadores e funções objetivo. Um modo de fazê-lo, que será usado ao longo deste texto, é: 
LEMA 19. Sejam $X^{\prime} \in(i$ dois subconjuntos fechados $\mathrm{em}$ um espaģo métrico. tal que $X^{\prime} \supset C^{\prime}$ e $X^{\prime}$ é compacto. $\delta(\cdot)$ um separador $\mathrm{em} . \mathrm{X}^{\prime} \sim G^{\prime}$

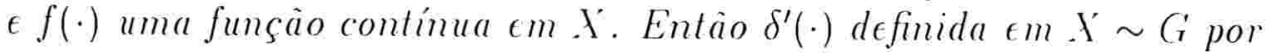

$$
\delta^{\prime}(x)=\inf _{z \in X}\{d(z, x) \mid f(z) \leqq f(x)-\delta(x)\}
$$

é um separador.

Prova. Como $\delta(\cdot)>0$ em $X \sim G$ e $f(\cdot)$ é continua, $\delta^{\prime}(\cdot)>0 \mathrm{em}$ $X \sim G$. Portanto, basta analisar as seqüências

$$
x^{i} \rightarrow x^{\infty} \text { e } \delta^{\prime}\left(x^{i}\right) \rightarrow 0
$$

Claramente existe um $N$ tal que, para todo $i>N, \delta^{\prime}\left(x^{i}\right)<\infty$; como $X$ é compacto e $f(\cdot)$ contínua, existe $\bar{x}^{i} \in X$ que realiza o ínfimo da definição de $\delta^{\prime}(\cdot)$, ou seja

$$
\delta^{\prime}\left(x^{i}\right)=d\left(\bar{x}^{i}, x^{i}\right) \text { e } f\left(\bar{x}^{i}\right) \leqq f\left(x^{i}\right)-\delta\left(x^{i}\right) .
$$

Logo, para todo $i>N$,

$$
\begin{gathered}
\delta^{\prime}\left(x^{i}\right)=d\left(\bar{x}^{i}, x^{i}\right) \rightarrow 0 \Rightarrow \bar{x}^{i} \rightarrow x^{\infty} \\
f\left(\bar{x}^{i}\right) \leqq f\left(x^{i}\right)-\delta\left(x^{i}\right) \Leftrightarrow \delta\left(x^{i}\right) \leqq f\left(x^{i}\right)-f\left(\bar{x}^{i}\right) .
\end{gathered}
$$

Como $f(\cdot)$ é contínua, os dois fatos acima implicam que $\delta\left(x^{i}\right) \rightarrow 0$.

Assim, uma vez que $\delta(\cdot)$ é um separador, segue que $x^{\infty} \in G$.

O resultado acima permite afirmar:

Lema 20. Sejam X e $G$ dois subconjuntos fechados em um espaço métrico, tal que $X^{\prime} \supset G, \delta(\cdot)$ um separador $\epsilon m X \sim G \in f(\cdot)$ uma funçâo contínua em $X$. Se uma seqüência é gerada em $X \sim G$ com a propriedade

$$
f\left(x^{i+1}\right) \leqq f\left(x^{i}\right)-\delta\left(x^{i}\right)
$$

então todo ponto de acumulação desta seqüência pertence a $G$.

Prova. Tome $\left(x^{j_{t}}\right)_{i \in \mathbf{N}}$ uma subseqüência convergente a $x^{\infty}$. Basta observar que

$$
\begin{gathered}
f\left(x^{j_{t+1}}\right) \leqq f\left(x^{j_{t}+1}\right) \leqq f\left(x^{j_{t}}\right)-\delta\left(x^{j_{t}}\right) \Rightarrow \\
\delta\left(x^{j_{t}}\right) \leqq f\left(x^{j_{t}}\right)-f\left(x^{j_{i+1}}\right) \rightarrow 0 \quad[f(\cdot) \text { é continua }]
\end{gathered}
$$

E, como $\delta(\cdot)$ é um separador, obtém-se $x^{\infty} \in G$.

O lema anterior sugere que um separador também pode ser visto como a medida de quanto deve cair a função objetivo para se ter a certeza de que todo ponto de acumulação da seqüência gerada está no conjunto alvo. Pode-se notar que a procura deste tipo de propriedade é comum em análise de convergência, como em [4], p. 32. onde o autor 
afirma que uma das principais idéias para a análise de convergência é garantir que a melhora entre as interaçòes é "grande" próximo a pontos nào-estacionários. Este resultado sugere um método para soluçòes de problemas de otimizaçào.

\section{Método de Otimização Baseado em Separadores - MOBS}

Dado um problema de minimizaçào: sejam $G$ o conjunte de soluçôes do $P M \in X$ um conjunto contendo $G$. Se $G$ e $X^{\prime}$ sào frchados $e \delta(\cdot)$ é um separador $\mathrm{em} . \mathrm{I}^{\prime} \sim G$ :

1. Inicialização: Tome $x^{1} \in X$ qualquer;

2. Iteraçâo: Se $x^{i} \in G$ pare;

Senão calcule $x^{i+1} \in X$ tal que

$$
f\left(x^{i+1}\right) \leqq f\left(x^{i}\right)-\delta\left(x^{i}\right)
$$

É importante destacar que o MOBS não está bem definido para todo separador. Isto é, dependendo da escolha do separador, pode não ser possível a obtenção de um próximo ponto que obedeça à condição do passo iterativo ${ }^{1}$.

TeOrema 21. Se $f(\cdot)$ for uma função contínua e a seqüência gerada pelo MOBS admitir algum ponto de acumulação então ele estará em $G$.

Prova. O teorema segue do lema 20.

Outra maneira de entender a prova acima é observar que o MOBS reproduz o $\mathrm{AMPC}$ para o separador $\delta^{\prime}(\cdot)$ dado pelo lema 19.

No artigo Generalized cutting plane algorithms este método não foi explicitamente formulado, uma vez que, no contexto apresentado, ele não era de maior relevância. Em seu trabalho, Eaves e Zangwill discutiram algoritmos de plano de corte, provando que muitos dos algoritmos conhecidos até 1971 eram casos particulares do AMPC. O objetivo do artigo era apresentar critérios para que parte dos cortes obtidos em etapas intermediárias pudessem ser eliminados. Isto é muito importante, pois nos algoritmos tradicionais o poliedro que aproximava o conjunto alvo aumentava de complexidade, ganhando um novo hiperplano a cada iteração. As estratégias desenvolvidas para a eliminação de alguns cortes se baseavam em parte na queda da função objetivo, da maneira como é exigida no MOBS. Assim o MOBS, dentro do contexto de algoritmos de planos de corte pode ser visto como um algoritmo forte,

\footnotetext{
${ }^{1}$ Um exemplo óbvio é tomar um separador constante. por exemplo, $\delta(x)=1$.
} 
onde a cada iteraşào é suficiente considerar. implicitamente. apenas o corte associado ao último ponto computado. 


\section{C'APíTLLO 4}

\section{Métodos de Ponto Proximal Baseados em Normas}

Neste capítulo será estudada uma classe de métodos proximais para a solução de problemas convexos. A origem destes algoritmos nos remete aos trabalhos de Minty $[\mathbf{1 5}, \mathbf{1 6}]$ e, dentro do contexto de programação matemática, aos artigos de Moreau[17] e Rockafellar [20, 19]. Estes trabalhos chamaram a atençào da comunidade de otimização para os métodos de ponto proximal, com destaque para sua ligação com métodos de Lagrangianos aumentados ${ }^{1}$.

\section{Ponto Proximal com Norma}

De uma maneira geral os métodos de programaçào convexa conhecidos como métodos de ponto proximal se encaixam no modelo abaixo:

Método de Ponto Proximal (MPP) Dada uma funçâo objetivo convexa $f(\cdot)$ e um conjunto convexo de pontos viáveis $V$, o MPP será definido por:

1. Inicialização: Tome qualquer $x^{1} \in V$;

2. Iteração: Calcule $x^{i+1}$ por

$$
x^{i+1} \in \underset{x \in V}{\operatorname{argmin}}\left\{f_{i}(x)=f(x)+\lambda_{i} r\left(x, x^{i}\right)\right\} .
$$

onde $r(\cdot, \cdot)$ é chamada de função de regularização $e\left(\lambda_{i}\right)_{i \in \mathbb{N}}$ é uma seqüencia dada de reais positivos.

O método proposto por Rockafellar em [20] usava como função de regularização

$$
r\left(x, x^{i}\right)=\left\|x-x^{i}\right\|_{2}^{2} .
$$

Nesse artigo é provada a convergência da seqüência gerada a uma solução de um problema de minimização irrestrita com função objetivo convexa, própria e semi-contínua inferior. Esta regularização será referenciada como regularização Euclidiana.

Nesta seção apresentar-se-ão os resultados centrais desta dissertação. Será proposta uma nova classe de métodos de ponto proximal

\footnotetext{
${ }^{1}$ Métodos de Lagrangianos aumentados são muito importantes em programação matemática, permitindo que problemas restritos possam ser resolvidos através da solução de uma seqüencia de problemas irrestritos. Ver, por exemplo, [3, 4].
} 
que estende o método baseado na regularização Euclidiana. porém. que tem convergência provada para uma família um pouco mais restrita de problemas. Isto é possivel graças ao uso da idéia de separadores apresentada no capítulo:3.

Primeiramente, deve-se destacar que a funçào objetivo leva a valores nos reais estendidos. Isto é especialmente interessante por permitir a manipulação de problemas restritos, através de sua conversão a um problema irrestrito.

Os problemas que serào tratados obedecem a algumas hipóteses técnicas que são comuns em otimização. Inicialmente, serão considerados problemas irrestritos. Isto nào reduz muito o tipo de problema estudado, como foi destacado no parágrafo anterior. A função objetivo é semi-contínua inferior. Esta hipótese está ligada à propriedade do mapa de subgradientes de funções convexas s.c.i. ser fechado, como foi visto no capítrilo 2. Supõe-se ainda que a função é contínua no domínio efetivo, pois procurar-se-á mostrar que o método proposto pode ser visto como caso particular do método de otimização baseado em separadores (MOBS), apresentado no final do capítulo 3. Por fim considera-se que o problema de otimização não é ilimitado (isto é, o valor ótimo não é menos infinito).

Hipótese 1. A função objetivo, $f: \mathbb{R}^{n} \mapsto \mathbb{R} \cup\{\infty\}$, é convexa, própria, semi-contínua inferior, contínua no domínio efetivo e limitada inferiormente. O conjunto viável é o $\mathbb{R}^{n}$, ou seja o problema é do tipo irrestrito.

Para solução de problemas convexos que obedecem a hipótese acima provar-se-á que é possível utilizar uma família de métodos de ponto proximal baseados em normas, que é descrita de maneira geral por:

Método de Ponto Proximal com norma (MPPN) Stja \|·\| uma norma qualquer, o MPPN será definido por:

1. Inicialização: Tome qualquer $x^{1} \in \operatorname{dom} f$;

2. Passo iterativo: Calcule $x^{i+1}$ por

$$
x^{i+1} \in \underset{x \in \mathbf{R}^{n}}{\operatorname{argmin}}\left\{f_{i}(x)=f(x)+\lambda_{i} r\left(x, x^{i}\right)\right\} .
$$

onde $r\left(x, x^{i}\right)=\phi\left(\left\|x-x^{i}\right\|\right) \in \phi(\cdot)$ é uma funçào tal que:

- $\phi: A \mapsto \mathbb{R}$, onde $A$ é um aberto contendo o $\mathbb{R}_{+}$, é continuamente diferenciável;

- $\phi^{\prime}(0)=0$;

- $\phi^{\prime}(\cdot)$ é estritamente crescente $\in m A^{2}$.

$e\left(\lambda_{i}\right)_{i \in \mathbf{N}} \dot{e}$ uma seqüência estritamente positiva $\epsilon$ limitada superiormente por $\tilde{\lambda}$. 
C'onstantemente usar-se-á o fato das funçòes $f_{i}(\cdot)$. que sào minimizadas a cada iteraçào. serem somas de funçòes que obedecem às condiçòes dos teoremas 16 e 1T. logo seus subgradientes podem ser calculados através da regra da soma e da cadeia dadas por esses teoremas. Ainda, se a norma escolhida for diferenciável basta usar o caso particular da regra de soma provado no teorema 1.5 e a regra da cadeia para funções diferenciáveis.

Deve ser notado que a classe de funçòes de regularizaçào proposta generaliza a regularização Euclidiana em dois sentidos. Primeiro. podese usar qualquer norma do $\mathbb{R}^{n}$, e, segundo, pode-se usar uma função estritamente convexa e diferenciável qualquer para a composição, com o cuidado que sua derivada no zero seja zero.

Algumas funçòes estritamente convexas no $\mathbb{R}_{+}$que podem ser usadas na composiçào com normas são:

- $\phi(x)=x^{\alpha}, a>1$

- $\phi(x)=x \ln (x+1)$;

- $\phi(x)=\cosh (x)$ (cosseno hiperbólico de $x)$;

- Seja $g(\cdot)$ uma função estritamente-convexa continuamente diferenciável, definida em um aberto contendo o $\mathbb{R}_{+}$. Tome $\phi(x)=$ $g(x)-g^{\prime}(0) x$.

Neste ponto, é conveniente discutir brevemente sobre as hipóteses impostas sobre $\phi(\cdot)$.

A hipótese que $\phi(\cdot)$ é continuamente diferenciável e estritamente convexa pode ser defendida pelo argumento que os problemas que devem ser resolvidos a cada iteração devem ser mais simples do que o problema original. Aqui a convexidade estrita desempenha papel de regularização do problema, eliminando comportamentos indesejados como a existência de múltiplas soluções ou de hessianas semi-definidas.

A condição $\phi^{\prime}(0)=0$ tem sua utilidade destacada no lema 23, que mostra que o algoritmo tem como pontos fixos as soluções do problema original.

1.1. Análise de convergência do MPPN. A afirmaçào mais importante que pode ser feita sobre um algoritmo de otimização é sobre a convergência, em algum sentido, da seqüência gerada a uma solução do problema considerado. O restante desta seçào dedicar-se-á a esta questão. Para isto, retoma-se o MOBS, descrito no final do capítulo 3. É possível provar que a família de métodos proximais proposta é um caso particular do MOBS.

\footnotetext{
${ }^{2}$ A função $\phi(\cdot)$, sob estas condiçòes, é claramente estritamente convexa em $\mathbb{R}_{+}$ e atinge mínimo no 0 .
} 
Antes de estudar a convergência do MPPN. apresentar-se-á um resultado simples. mas que será várias vezes usado nas manipulaçòes seguintes.

LEMA 21. Ś jam $f(\cdot) \in g(\cdot)$ duas funçós de $\mathbb{R}^{n} \in m \mathbb{R} \cup\{\infty\}$. tais que

$$
f(x)=g(x-a)
$$

então

$$
\gamma \in \partial f(\bar{x}) \Leftrightarrow \gamma \in \partial g(\bar{x}-a)
$$

Prova.

$$
\begin{gathered}
\gamma \in \partial f(\bar{x}) \Leftrightarrow \\
\forall x \in \mathbb{R}^{n}, \quad f(x) \geqq f(\bar{x})+\langle\gamma, x-\bar{x}\rangle \Leftrightarrow \\
\forall x \in \mathbb{R}^{n}, \quad g(x-a) \geqq g(\bar{x}-a)+\langle\gamma, x-\bar{x}\rangle \Leftrightarrow \\
\forall y \in \mathbb{R}^{n}, \quad g(y) \geqq g(\bar{x}-a)+\langle\gamma, y+a-\bar{x}\rangle .
\end{gathered}
$$

Após este lema técnico passa-se a análise de convergência do MPPN. Primeiramente deve-se mostrar que o método está bem definido, isto é, que dado o ponto $x^{i}$, o iterado $x^{i+1}$ existe e é único.

LEMA 22. O método MPPN está bem definido.

Prova. A prova está dividida em 3 partes. As duas primeiras garantem a existência de $x^{i+1}$, a seguinte sua unicidade.

- os conjuntos de nível de $r\left(\cdot, x^{i}\right)=\phi\left(\left\|\cdot-x^{i}\right\|\right)$ são compactos.

Obviamente os conjuntos de nível

$$
C(k)=\left\{x \mid r\left(x, x^{i}\right) \leqq k\right\}
$$

são fechados, pois a função $r\left(\cdot, x^{i}\right)$ é contínua. Portanto, para provar a compacidade dos conjuntos de nível, basta mostrar que eles são limitados.

Observa-se, pelo teorema fundamental do cálculo, que:

$$
r\left(x, x^{i}\right)-r\left(x^{i}, x^{i}\right)=o\left(\left\|x-x^{i}\right\|\right)-\phi(0)=\int_{0}^{\left\|x-x^{i}\right\|} \phi^{\prime}(t) d t
$$


Se $\left\|x-x^{i}\right\| \geqq 1$. vale:

$$
\begin{aligned}
r\left(x . x^{i}\right) & =\int_{0}^{\left\|r-x^{2}\right\|} \phi^{\prime}(t) d t+\phi(0) \\
& =\int_{1}^{\mid r-x^{2} \|} \phi^{\prime}(t) d t+\int_{0}^{1} \phi^{\prime}(t) d t+\phi(0) \\
& \geqq o^{\prime}(1)\left(\left\|x-x^{i}\right\|-1\right)+\int_{0}^{1} \phi^{\prime}(t) d t+\phi(0) \\
& =o^{\prime}(1)\left\|x-x^{i}\right\|-\phi^{\prime}(1)+\phi(1) \\
& \geqq \phi^{\prime}(1)\|x\|-\phi^{\prime}(1)\left\|x^{i}\right\|-\phi^{\prime}(1)+\phi(1) \\
& =\delta^{\prime}(1)\|x\|-\phi^{\prime}(1)\left(\left\|x^{i}\right\|+1\right)+\phi(1) \\
& =\phi^{\prime}(1)\|x\|+K^{i},
\end{aligned}
$$

onde $K^{i}$. para um dado $x^{i}$, é uma constante. A primeira desigualdade segue do fato de $\phi^{\prime}(\cdot)$ ser crescente e a segunda vem da desigualdade triangular.

Assim, dada uma constante $\mathrm{k}$ :

$$
r\left(x, x^{i}\right) \leqq k \Rightarrow\left\|x-x^{i}\right\| \leqq 1 \text { ou }\|x\| \leqq \frac{k-K^{-i}}{\phi^{\prime}(1)}
$$

e se conclui que, qualquer que seja $k$, o conjunto de nível $C(k)$ é limitado.

- $f_{i}(\cdot)$ admite mínimo, isto é argmin $\operatorname{me}_{x \in \mathbf{R}^{n}}\left\{f_{i}(x)\right\} \neq \emptyset$.

Como, pela hipótese $1, f(\cdot)$ é limitada inferiormente, seja $l$ um limitante inferior de $f(\cdot)$. Observa-se que:

$$
f_{i}(x)=f(x)+\lambda_{i} r\left(x, x^{i}\right) \geqq l+\lambda_{i} r\left(x, x^{i}\right) .
$$

Logo $f_{i}(\cdot)$ possui conjuntos de nível limitados (uma vez que $r\left(\cdot, x^{i}\right)$ é assim pelo primeiro ítem). Como $f_{i}(\cdot)$ é semi-contínua inferior, seus conjuntos de nível são fechados e desta forma a minimizaçào do passo iterativo pode ser restrita a um compacto. Conclui-se a existência de mínimo.

- $r\left(\cdot, x^{i}\right)$ é estritamente convexa.

Seja $\lambda \in(0.1)$ e $x \neq y$,

$$
\begin{aligned}
r\left(\lambda x+(1-\lambda) y, x^{i}\right) & =\phi\left(\left\|\lambda\left(x-x^{i}\right)+(1-\lambda)\left(y-x^{i}\right)\right\|\right) \\
& \leqq \phi\left(\lambda\left\|x-x^{i}\right\|+(1-\lambda)\left\|y-x^{i}\right\|\right) \\
& <\lambda \phi\left(\left\|x-x^{i}\right\|\right)+(1-\lambda) \phi\left(\left\|y-x^{i}\right\|\right) .
\end{aligned}
$$


A primeira desigualdade segue do fato de $o(\cdot)$ ser crescente no $\mathbb{R}_{+}$(tem derivada nào-negativa) e a segunda do fato de $o(\cdot)$ ser estritamente convexa.

- $f_{i}(\cdot)$ admite mínimo único.

Como $f_{i}(\cdot)$ é soma de uma função convexa com uma funçào estritamente convexa, $f_{i}(\cdot)$ é estritamente convexa e segue a unicidade do mínimo.

Deve-se destacar neste ponto que o lema anterior diz que, dado $\lambda_{i}$, o iterado $x^{i+1}$ é função do iterado anterior. Isto será usado na definição do separador associado ao MPPN. Para chamar a atenção a este fato passar-se-á a usar, como abuso de notação

$$
x^{i+1}=\underset{x \in \mathbf{R}^{n}}{\operatorname{argmin}}\left\{f_{i}(x)=f(x)+\lambda_{i} r\left(x, x^{i}\right)\right\} .
$$

O próximo fato mostra o que acontece quando o método para em "tempo" finito. Isto é, quando $x^{i+1}=x^{i}$.

LEMA 23. O método MPPN para no ponto $x^{i}$ se, $\epsilon$ só se, $x^{i} \dot{e}$ solução da minimizaçâo de $f(\cdot)$.

Prova.

$$
\begin{aligned}
\text { O método para } & \Leftrightarrow x^{i}=\underset{x \in \mathbb{R}^{n}}{\operatorname{argmin}}\left\{f(x)+\lambda_{i} r\left(x, x^{i}\right)\right\} \\
& \Leftrightarrow \exists \gamma_{f}^{i} \in \partial f\left(x^{i}\right), \gamma_{r}^{i} \in \partial r\left(x^{i}, x^{i}\right)^{3}: \gamma_{f}^{i}+\lambda_{i} \gamma_{r}^{i}=0 \\
& \Leftrightarrow \exists \gamma_{f}^{i} \in \partial f\left(x^{i}\right): \gamma_{f}^{i}=0 \\
& {\left[\partial r\left(x^{i}, x^{i}\right)=\phi^{\prime}(\|0\|) \partial(\|\cdot\|)(0)=\{0\}\right] } \\
& \Leftrightarrow x^{i} \text { é solução da minimização de } f(\cdot) .
\end{aligned}
$$

De posse do resultado acima, é razoável questionar se o MPPN pode convergir em tempo finito ou não. Sabe-se, do artigo de Rockafellar [20], que o algoritmo baseado no quadrado da norma Euclidiana converge em um número finito de passos para uma classe especial de problemas, que engloba problemas de minimização irrestrita gerados a partir da transformaçào de um problema linear. Porém, no caso da função $f(\cdot)$ ser diferenciável em todo o $\mathbb{R}^{n}$ (logo finita em todo espaço),

\footnotetext{
${ }^{3}$ Se $\gamma$ é um subgradiente da função $r\left(\cdot, x^{i}\right)$ calculado no ponto $\bar{x}$, a notação mais precisa seria: $\gamma \in \partial\left(r\left(\cdot, x^{i}\right)\right)(\bar{x})$. Porém, prefere-se a notação $\gamma \in \partial r\left(\bar{x}, x^{i}\right)$, que é mais limpa.
} 
uma pequena manipulaçào mostra que o método para em tempo finito se, e só se. o primeiro ponto for uma soluçào.

$$
\begin{gathered}
x^{i+1}=\underset{r \in \mathbf{R}^{n}}{\operatorname{argmin}}\left\{f(x)+\lambda_{i} O\left(\left\|x-x^{i}\right\|\right)\right\} \Leftrightarrow \\
\nabla f\left(x^{i+1}\right)+\lambda_{i} \phi^{\prime}\left(\left\|x^{i+1}-x^{i}\right\|\right) \partial(\|\cdot\|)\left(x^{i+1}-x^{i}\right)=0 \Leftrightarrow \\
\nabla f\left(x^{i+1}\right)=\lambda_{i} \phi^{\prime}\left(\left\|x^{i+1}-x^{i}\right\|\right) \partial(\|\cdot\|)\left(x^{i+1}-x^{i}\right) .
\end{gathered}
$$

O lema anterior diz que. se $x^{i}$ não é uma soluçào, entào $x^{i+1} \neq x^{i}$. Deste modo conclui-se que o iterado gerado a partir de um ponto que nào é solução terá gradiente não nulo e. conseqüentemente. não será uma solução da minimização irrestrita de $f(\cdot)$.

Busca-se mostrar que o MPPN é um caso particular do MOBS, isto é, que a cada passo a função objetivo cai pelo menos o valor dado por um separador. Portanto, deve-se tentar avaliar de quanto cai a função objetivo a cada passo. Para que isto seja possivel. usar-se-á fortemente as hipóteses de convexidade.

Em primeiro lugar, como $f(\cdot)$ é convexa, sabe-se que

$$
f\left(x^{i}\right) \geqq f\left(x^{i+1}\right)+\left\langle\gamma_{f}, x^{i}-x^{i+1}\right\rangle, \forall \gamma_{f} \in \partial f\left(x^{i+1}\right) .
$$

Logo, qualquer medida do valor do produto interno que aparece na expressão acima pode servir para avaliar a queda da função objetivo.

Para tal, usar-se o fato de $f_{i}(\cdot)$ obedecer às hipóteses dos teoremas de regra da cadeia e regra da soma para subdiferenciais. Mais ainda, utiliza-se que $f_{i}(\cdot)$ é minimizada a cada iteração e que $r\left(\cdot, x^{i}\right)$ e $\phi(\|\cdot\|)$ obedecem às condições do lema 21. Assim pode-se afirmar que:

$$
\begin{gathered}
0 \in \partial f_{i}\left(x^{i+1}\right) \Leftrightarrow \quad \text { [Pelo lema 17] } \\
\exists \gamma_{f} \in \partial f\left(x^{i+1}\right) \text { e } \gamma_{r} \in \partial r\left(x^{i+1}, x^{i}\right): \gamma_{f}+\lambda_{i} \gamma_{r}=0 \Leftrightarrow \\
\exists \gamma_{f} \in \partial f\left(x^{i+1}\right) \text { e } \gamma_{N} \in \partial(\|\cdot\|)\left(x^{i+1}-x^{i}\right): \\
\gamma_{f}+\lambda_{i} \phi^{\prime}\left(\left\|x^{i+1}-x^{i}\right\|\right) \gamma_{N}=0 .
\end{gathered}
$$

Estas últimas expressões apresentam boas alternativas para a avaliação do valor do produto interno presente na equação 14 . Isto pode ser confirmado pelas manipulações abaixo. Substituindo este resultado na expressão 14 , tem-se

$$
\begin{aligned}
\exists \gamma_{N} \in \partial(\|\cdot\|) & \left(x^{i+1}-x^{i}\right): \\
f\left(x^{i}\right) & \geqq f\left(x^{i+1}\right)+\lambda_{i} \phi^{\prime}\left(\left\|x^{i+1}-x^{i}\right\|\right)\left\langle\gamma_{N}, x^{i+1}-x^{i}\right\rangle \\
& \geqq f\left(x^{i+1}\right)+\lambda_{i} \phi^{\prime}\left(\left\|x^{i+1}-x^{i}\right\|\right)\left\|x^{i+1}-x^{i}\right\| .
\end{aligned}
$$

A última desigualdade segue do fato de $\gamma_{N}$ ser um subgradiente da norma em $x^{i+1}-x^{i}$. 
Deste modo. é possivel considerar como um candidato a separador a função:

$$
\lambda_{i} \phi^{\prime}\left(\left\|x^{i+1}-x^{i}\right\|\right)\left\|x^{i+1}-x^{i}\right\| .
$$

Mais uma vez destaca-se que o lema 22 (que afirma que o método está bem definido) mostra que o iterado $x^{i+1}$ é função de $x^{i}$ e $\lambda_{i}$. Assim, infelizmente, o expressão acima ainda nào é uma função apenas de $x^{i}$. Para contornar este fato utiliza-se o limitante superior da seqüência $\left(\lambda_{i}\right)_{i \in \mathbb{N}}$ dado na definição do método, $\tilde{\lambda}$.

LEMA 24. Seja $\tilde{\lambda}$ o limitante superior de $\left(\lambda^{i}\right)_{i \in \mathbf{N}}$ dado na definiçâo do $M P P N$. Definindo $\delta: \mathbb{R}^{n} \sim G \mapsto \mathbb{R}$ por:

$$
\delta(x)=\widetilde{\lambda} \phi^{\prime}\left(\| \widetilde{x}-x \eta_{1}\right)\|\widetilde{x}-x\|,
$$

onde

$$
\widetilde{x}=\underset{z \in \mathbb{R}^{n}}{\operatorname{argmin}}\{f(z)+\widetilde{\lambda} r(z, x)\} .
$$

tem-se que $\delta(\cdot)$ é um separador em relação ao conjunto

$$
G=\underset{x \in \mathbb{R}^{n}}{\operatorname{argmin}}\{f(x)\},
$$

que é fechado pelo corolário \%.

Prova. Primeiramente, nota-se que, pelo lema $22, \widetilde{x}$ está bem definido. Também, pelas hipóteses válidas para $\phi(\cdot), \delta(x)>0$, para todo $x \notin G$.

Para provar que $\delta(\cdot)$ é um separador, consideram-se seqüências $x^{i} \rightarrow$ $x^{\infty}$ e $\delta\left(x^{i}\right) \rightarrow 0$. Para esta análise é preciso retomar as manipulações que motivaram a definição de $\delta(\cdot)$ :

$$
\widetilde{x}=\underset{z \in \mathbb{R}^{n}}{\operatorname{argmin}}\{f(z)+\widetilde{\lambda} r(z, x)\} \Leftrightarrow
$$

$$
\exists \gamma_{f} \in \partial f(\widetilde{x}), \gamma_{r} \in \partial r(\widetilde{x}, x): \gamma_{f}+\widetilde{\lambda} \gamma_{r}=0 \Leftrightarrow
$$

(17) $\exists \gamma_{f} \in \partial f(\widetilde{x}), \gamma_{N} \in \partial(\|\cdot\|)(\widetilde{x}-x): \gamma_{f}+\widetilde{\lambda} \phi^{\prime}(\|\widetilde{x}-x\|) \gamma_{N}=0$.

A prova será apresentada em três partes:

- A seqüência $\left(\widetilde{x}^{i}\right)_{i \in \mathbb{N}} \rightarrow x^{\infty}$.

Supõe-se, por contradição, que a afirmação não é válida. Então existe um $\epsilon>0$ e uma subseqüência de índices $j_{i}$ tais que:

$$
\begin{gathered}
\left\|\tilde{x}^{j_{i}}-x^{j_{i}}\right\|>\epsilon \Rightarrow \\
\delta\left(x^{j_{i}}\right)=\widetilde{\lambda} \phi^{\prime}\left(\left\|\widetilde{x}^{j_{i}}-x^{j_{i}}\right\|\right)\left\|\tilde{x}^{j_{t}}-x^{j_{i}}\right\|>\widetilde{\lambda} \phi^{\prime}(\epsilon) \epsilon>0 .
\end{gathered}
$$


contrariando a convergência de $\delta(\cdot)$.

- Sejam seqüencias $\left(\gamma_{f}^{i}\right)_{i \in \mathbb{N}}$ e $\left(\gamma_{i}^{i}\right)_{i \in \mathbb{N}}$ associadas a $\left(x^{i}\right)_{i \in \mathbb{N}}$ que obedecem a relaçào da equaçào 16 . Entào $\gamma_{j}^{i}=-\tilde{\lambda}_{\gamma_{r}}^{i} \rightarrow 0$.

Sabemos, pelo ítem anterior que

$$
\widetilde{x}^{i}-x^{i} \rightarrow 0 .
$$

Logo, como $\gamma_{r}^{i} \in \partial \phi(\|\cdot\|)\left(\widetilde{x}^{i}-x^{i}\right)$, sabemos, pelo teorema 14 que a seqüência é limitada.

Seja $\gamma^{\infty}$ um ponto de acumulação de $\left(\gamma_{f}^{i}\right)_{i \in \mathbf{N}}$. Então

$$
\begin{aligned}
& \left\{\begin{array}{l}
\tilde{x}^{j_{t}}-x^{j_{t}} \rightarrow 0 \\
\gamma_{r}^{j_{i}} \rightarrow-\frac{\gamma^{\infty}}{\tilde{\hat{j}}} \\
\gamma_{r}^{j_{i}} \in \partial \phi(\|\cdot\|)\left(\widetilde{x}^{j_{i}}-x^{j_{1}}\right)
\end{array}\right\} \Rightarrow-\frac{\gamma^{\infty}}{\widetilde{\lambda}} \in \phi^{\prime}(\|0\|) \partial(\|\cdot\|)(0) \Rightarrow \\
& \gamma^{\infty}=0 .
\end{aligned}
$$

Onde a primeira implicação é conseqüência do fato do mapa subdiferencial de uma função contínua ser fechado (lema 12).

- $\delta(\cdot)$ é um separador.

Usando as definições e fatos provados nos ítens anteriores:

$$
\left\{\begin{array}{l}
x^{i} \rightarrow x^{\infty} \\
\delta\left(x^{i}\right) \rightarrow 0
\end{array}\right\} \Rightarrow\left\{\begin{array}{l}
\tilde{x}^{i} \rightarrow x^{\infty} \\
\gamma_{f}^{i} \rightarrow 0 \\
\gamma_{f}^{i} \in \partial f\left(\widetilde{x}^{i}\right)
\end{array}\right\} \Rightarrow 0 \in \partial f\left(x^{\infty}\right) .
$$

uma vez que o mapa subdiferencial de $f(\cdot)$ é fechado. Por fim, a última relação vale se, e só se, $x^{\infty} \in G$.

Para se poder usar os resultados de convergência do MOBS ao MPPN, é preciso provar que a cada iteração a função objetivo cai pelo menos o valor dado pelo separador do lema 24. Observando a dedução que levou a definição do separador, nota-se que há apenas uma dificuldade técnica a ser superada: a substituição de $\lambda_{i}$ por $\widetilde{\lambda}$ (feita para que o separador não dependesse de $i$ ). Lembrando que $\tilde{\lambda} \geqq \lambda_{i}$, podese dizer intuitivamente que o ponto $\widetilde{x}$ calculado no separador é uma aproximação pessimista de $x^{i+1}$, uma vez que o peso dado à regularização foi maior, penalizando o afastamento de $x^{i}$. Esta observação é formalizada por:

Lema 25. A seqüência gerada pelo MPPN tem a propriedade

$$
f\left(x^{i+1}\right) \leqq f\left(x^{i}\right)-\delta\left(x^{i}\right)
$$

onde $\delta(\cdot)$ é o separador definido no lema 24. 
Prova. A prova divide-se em duas partes:

- $f\left(\tilde{x}^{i}\right) \leqq f\left(x^{i}\right)-\delta\left(x^{i}\right)$.

Mais uma vez. sejam seqüencias $\left(\gamma_{j}^{i}\right)_{i \in \mathbf{N}}$ e $\left(\gamma_{i}^{i}\right)_{i \in \mathbf{N}}$ associadas a $\left(x^{i}\right)_{i \in \mathbf{N}}$ que obedecem a relaçào da equaçào 16 . Observe que:

$$
\begin{aligned}
& \left.f\left(x^{i}\right) \geqq f\left(\tilde{x}^{i}\right)+\left\langle\hat{i}_{f}^{i}, x^{i}-\widetilde{x}^{i}\right\rangle \quad \text { [Convex. da } f(\cdot)\right] \\
& =f\left(\tilde{x}^{i}\right)+\tilde{\lambda}\left\langle\gamma_{r}^{i}, \tilde{x}^{i}-x^{i}\right\rangle \quad \text { [Equação 16] } \\
& =f\left(\tilde{x}^{i}\right)+\tilde{\lambda} \phi^{\prime}\left(\left\|\tilde{x}^{i}-x^{i}\right\|\right)\left\langle\gamma_{N}^{i}, \widetilde{x}^{i}-x^{i}\right\rangle \quad[\text { Equação 17] } \\
& \geqq f\left(\tilde{x}^{i}\right)+\tilde{\lambda} \phi^{\prime}\left(\left\|\tilde{x}^{i}-x^{i}\right\|\right)\left\|\tilde{x}^{i}-x^{i}\right\| \quad\left[\hat{\imath}^{i} \in \partial(\|\cdot\|)\left(\tilde{x}^{i}-x^{i}\right)\right] \\
& \left.=f\left(\tilde{x}^{i}\right)+\delta\left(x^{i}\right) \quad \text { [Def. de } \delta(\cdot)\right] \text {. }
\end{aligned}
$$

- $f\left(x^{i+1}\right) \leqq f\left(\tilde{x}^{i}\right)$. Claramente, pelas definiçòes de $x^{i+1}$ e $\tilde{x}^{i}$, há necessidade de tratar apenas o caso $\tilde{\lambda}>\lambda_{i}$.

Sabe-se que

$$
\begin{aligned}
f\left(\tilde{x}^{i}\right)+\tilde{\lambda} r\left(\tilde{x}^{i}, x^{i}\right) & \leqq f\left(x^{i+1}\right)+\tilde{\lambda} r\left(x^{i+1}, x^{i}\right) & & \text { [Def. de } \left.\tilde{x}^{i}\right] \\
-f\left(\widetilde{x}^{i}\right)-\lambda_{i} r\left(\widetilde{x}^{i}, x^{i}\right) & \leqq-f\left(x^{i+1}\right)-\lambda_{i} r\left(x^{i+1}, x^{i}\right) & & \text { [Def. de } \left.x^{i+1}\right]
\end{aligned}
$$

Somando as duas expressões imediatamente acima tem-se

$$
\begin{array}{rlrl}
\left(\tilde{\lambda}-\lambda_{i}\right) r\left(\tilde{x}^{i}, x^{i}\right) & \leqq\left(\tilde{\lambda}-\lambda_{i}\right) r\left(x^{i+1}, x^{i}\right) \Rightarrow & \\
r\left(\tilde{x}^{i}, x^{i}\right) & \leqq r\left(x^{i+1}, x^{i}\right) . \quad\left[\tilde{\lambda}>\lambda_{i}\right]
\end{array}
$$

Assim, pode-se escrever a relação

$$
\begin{aligned}
-\lambda_{i} r\left(x^{i+1}, x^{i}\right) & \leqq-\lambda_{i} r\left(\widetilde{x}^{i}, x^{i}\right) \\
f\left(x^{i+1}\right)+\lambda_{i} r\left(x^{i+1}, x^{i}\right) & \left.\leqq f\left(\widetilde{x}^{i}\right)+\lambda_{i} r\left(\widetilde{x}^{i}, x^{i}\right) \quad \text { [Def. de } x^{i+1}\right] \\
\text { que somadas dào a relaçào desejada. } &
\end{aligned}
$$

O resultado segue da união dos dois ítens.

Os últimos dois lemas mostram que o MPPN é um caso particular do MOBS. Graças a isto é possível apresentar o resultado central deste capítulo: o teorema de convergência do MPPN. baseado no teorema de convergência do MOBS.

TEOREMa 22. Todo ponto de acumulaçào da seqüência gerada pelo MPPN é uma solução da minimização irrestrita de $f(\cdot)$.

Prova. Segue do teorema de convergência do MOBS, lembrando da hipótese de que $f(\cdot)$ é contínua em seu domínio efetivo. 
O resultado acima é típico da análise de convergência de algoritmos em programaçào nào-linear. Porém nào está garanticla a existência de pontos de acumulaçào da seqüencia gerada e. deste modo. nào está garantida a obtençào de soluçòes. Isto leva diretamente a uma outra questão: quais sào as hipóteses necessárias para que a seqüência gerada pelo MPPN scja limitada, garantindo assim a existência de pontos de acumulaçào? Uma solução possivel, e usual em programaçào matemática, é supor que os conjuntos de nível da funçào objetivo, $f(\cdot)$. sào limitados. Como a seqüência gerada não aumenta a função objetivo, teríamos a limitação. Mostrar-se-á que outra soluçào é a adoção de uma norma conveniente que permita provar a limitação da seqüência gerada pelo MPPN.

1.2. Norma Euclidiana. Uma solução encontrada para a limitação da seqüência gerada pelo MPP baseado na regularizaçào Euclidiana usa o fato da seqüência gerada por ele ser Fejér monótona em relação ao conjunto de soluções da minimização de $f(\cdot)$. Esta propriedade é mantida quando compomos a norma Euclidiana com outras funções estritamente convexas.

Fazendo uma manipulação simples, semelhante à manipulação feita logo após o lema 22 , observa-se que

$$
\begin{gathered}
x^{i+1}=\underset{x \in \mathbf{R}^{n}}{\operatorname{argmin}}\left\{f(x)+\lambda_{i} \phi\left(\left\|x-x^{i}\right\|_{2}\right)\right\} \Leftrightarrow \\
\exists \gamma_{f}^{i} \in \partial f\left(x^{i+1}\right): \gamma_{f}^{i}+\lambda_{i} \phi^{\prime}\left(\left\|x^{i+1}-x^{i}\right\|_{2}\right) \nabla\left\|x^{i+1}-x^{i}\right\|_{2}=0 \Leftrightarrow \\
\gamma_{f}^{i}=\underbrace{\lambda_{i} \frac{\phi^{\prime}\left(\left\|x^{i+1}-x^{i}\right\|_{2}\right)}{\left\|x^{i+1}-x^{i}\right\|_{2}}}_{>0}\left(x^{i}-x^{i+1}\right) .
\end{gathered}
$$

Portanto. uma explicação geométrica para a Fejér monotonicidade é obtida da expressâo acima quando se considera o subespaço gerado por $\left\{x^{i}, x^{i+1}, g\right\}$, veja figura 1 . Neste subespaço traça-se o plano $H$ perpendicular ao subgradiente $\gamma_{f}^{i}$ de $f(\cdot)$ no ponto $x^{i+1}$ e passando por $x^{i+1}$. Como o subgradiente de $f(\cdot)$ considerado é um múltiplo positivo de $x^{i}-x^{i+1}$ (expressão 18), é possível provar, pela convexidade de $f(\cdot)$, que $g$ está do lado oposto ao de $x^{i}$. Dessa forma o ângulo $a=\widehat{x^{i} x^{i+1}} g$ deve ser maior do que o ângulo entre $x^{i}-x^{i+1}$ e $H$, que é de $90^{\circ}$. Portanto o lado oposto ao ângulo $\alpha$ deve ser o maior do triângulo dado por $x^{i}, x^{i+1}, g$.

Estes argumentos podem ser formalizados algebricamente. 


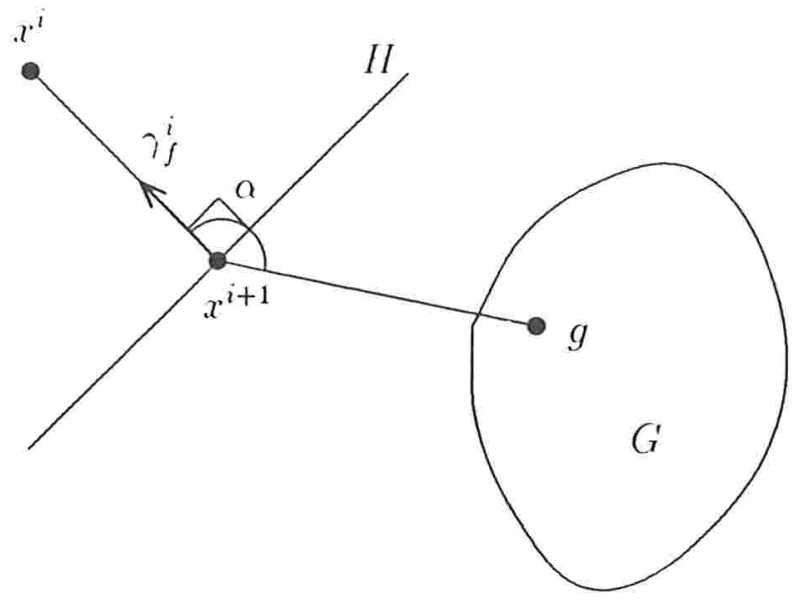

Figura 1. Féjer monotonicidade da seqüência gerada pelo MPP2.

LEMa 26. Se a norma utilizada é a Euclidiana entâo seqüência gerada pelo MPPN é Fejér monótona em relação ao conjunto $G=$ $\operatorname{argmin}_{x \in \mathbf{R}^{n}}\{f(x)\}$.

Prova. Supòe-se que $x^{i} \neq x^{i+1}$. Dado um ponto qualquer $g \in G$, pode-se ver, da equação 18 , da monotonicidade de $\partial f(\cdot)$ e do fato de $0 \in \partial f(g)$, que

$$
\left\langle x^{i}-x^{i+1}, x^{i+1}-g\right\rangle=\operatorname{cte}\left\langle\gamma_{f}^{i}, x^{i+1}-g\right\rangle \geqq 0 .
$$

Note que a equação 19 confirma os argumentos geométricos sobre os ângulos, bastando lembrar da relação do produto interno com a função cosseno.

Assim, tem-se

$$
\begin{aligned}
\left\|x^{i}-g\right\|_{2}^{2} & =\left\langle x^{i}-x^{i+1}+x^{i+1}-g, x^{i}-x^{i+1}+x^{i+1}-g\right\rangle \\
& =\left\|x^{i}-x^{i+1}\right\|_{2}^{2}+\left\|x^{i+1}-g\right\|_{2}^{2}+2\left\langle x^{i}-x^{i+1}, x^{i+1}-g\right\rangle \\
& \geqq\left\|x^{i+1}-g\right\|_{2}^{2} \quad \text { [Equação 19] }
\end{aligned}
$$

De posse deste resultado, pode-se enunciar um teorema de convergência (não apenas no sentido de pontos de acumulaçào como o teorema 22) para métodos de ponto proximal que usam a norma Euclidiana.

Teorema 23. Se o conjunto de soluçòes da minimizaçáo irrestrita de $f(\cdot)$ for não vazio, então o MPPN usando a norma Euclidiana converge a uma soluçào. 
Prova. Sabe-se. pelo teorema de convergência do MPPX. teorema 22. que todo ponto de acumulaçào da seqüência é ponto de Gi. Do lema 26. sabe-se que a seqüência é Fejér monótona em relaçào ao conjunto de soluções $G$. Como $G \neq \emptyset$, conclui-se que a seqüência é limitada, possuindo pontos de acumulação. Como todo limite de subseqüência estará em $G$, mais uma vez da Fejér monotonicidade. existirá um único ponto de acumulação.

\section{MPPN e problemas restritos}

Nas seçòes anteriores mostrou-se que o MPPN pode ser utilizado no tratamento da minimização irrestrita de uma funçào convexa. Porém o MPPN também se aplica a minimizações restritas. Considerar-se-á problemas que obedecem a hipóteses análogas às hipóteses assumidas no caso irrestrito.

Hipótese 2. A função objetivo $f(\cdot)$ é convexa, própria e semicontínua inferior. contínua no domínio efetivo e limitada inferiormente. O conjunto viável, $V$, é convexo e fechado ${ }^{4}$. Por fim, a intersecçào do domínio efetivo de $f(\cdot)$ e do conjunto viável é não vazia.

Isto é necessário para que o problema irrestrito equivalente, dado pelo lema 14, obedeça as condições para a convergência do MPPN.

Método de Ponto Proximal com norma para problemas restritos (MPPNR) Seja $\|\cdot\|$ uma norma qualquer. o MPPNR será definido por:

1. Inicializaçào: Tome qualquer $x^{1} \in V$;

2. Passo iterativo: Calcule $x^{i+1}$ por

$$
x^{i+1}=\underset{x \in V^{V}}{\operatorname{argmin}}\left\{f_{i}(x)=f(x)+\lambda_{i} r\left(x . x^{i}\right)\right\} .
$$

onde $r\left(x, x^{i}\right)=\phi\left(\left\|x-x^{i}\right\|\right) \in \phi(\cdot)$ obedece às condiçós descritas no MPPN. $\left(\lambda_{i}\right)_{i \in \mathbf{N}}$ é uma seqüência estritamente positiva e limitada superiormente por $\tilde{\lambda}$.

Pode-se provar, facilmente, que este método é convergente no mesmo sentido do MPPN.

TEOREma 24. Todo ponto de acumulaçào da seqüencia gerada pelo MPPNR é uma soluçào do problema de minimizar $f(\cdot)$ sujeito ao conjunto viável $\mathrm{V}$.

\footnotetext{
${ }^{4}$ Desta forma a funçào indicadora de $V$ é convexa e semi-contínua inferior
} 
Prova. O problema proposto é. pelo lema 14. equivalente ao problema de minimizaçào irrestrita de $f(\cdot)+\operatorname{ind}\left(\cdot \mid 1^{\cdot}\right)$. Este último pode ser resolvido pelo MPPN. O passo do MPPN é

$$
\underset{x \in \mathbb{R}^{n}}{\operatorname{argmin}}\left\{f_{i}(x)=f(x)+\operatorname{ind}(x \mid V)+\lambda_{i} r\left(x, x^{i}\right)\right\} .
$$

Que é equivalente a

$$
\underset{x \in V^{\prime}}{\operatorname{argmin}}\left\{f_{i}(x)=f(x)+\lambda_{i} r\left(x, x^{i}\right)\right\},
$$

pois

$$
f(x)+\operatorname{ind}(x \mid V)= \begin{cases}f(x), & \text { se } x \in \operatorname{dom} f \cap V \neq \emptyset \\ \infty, & \text { caso contrário. }\end{cases}
$$

Ainda, usando a norma Euclidiana, podé-se obter um teorema mais forte de convergência:

TeOrema 25. Se a norma utilizada no MPPNR for a Euclidiana então o MPPRN converge a uma solução do provilema de minimização de $f(\cdot)$ no conjunto viável $V$, caso o conjunto de soluçôes seja não vazio.

Prova. Basta lembrar que o MPPNR é um caso particular do MPPN e aplicar o teorema 23.

Por fim, pode-se destacar o que acontece se o MPPNR para em um número finito de passos.

LEMA 27. O método MPPNR para no ponto $x^{i}$ se. $\epsilon$ só se, $x^{i} e ́$ uma solução da minimizaçào de $f(\cdot)$ no conjunto viável $V$.

Prova. Como o MPPNR é um caso particular do MPPN. aplica-se o lema 23. 



\section{CAPíTULO 5}

\section{Métodos de Ponto Proximal Baseados em Funções de Bregman}

Neste capítulo serào discutidos métodos de ponto proximal que usam como regularizaçào funções com propriedades semelhantes a distâncias. mas que nào surgem a partir de normas como acontecia no MPPN. Um fato muito importante é que. caso funções de regularização adequadas sejam encontradas, pode-se substituir um problema restrito por uma seqüencia de problemas irrestritos, tornando o uso do método muito atraente. A classe de funçòes de regularização que será estudada neste capítulo são as distâncias de Bregman, introduzidas en $[5]$ c posteriormente utilizadas no MPP. ${ }^{1}$ Deve-se destacar que existe uma outra classe de funções, que nào será apresentada nesta dissertação, mas que é utilizada como regularização no método de ponto proximal: as $\varphi$-divergências. Este tipo de função é empregado quando o conjunto viável é o ortante positivo $\left(\mathbb{R}_{+}^{n}\right){ }^{2}$

\section{Distâncias de Bregman}

Outro tipo de funções de regularização que leva à convergência da seqüência gerada pelo MPP são as distâncias de Bregman. Elas foram introduzidas originalmente no trabalho [5] e sua conexão com o método de ponto proximal foi estudada em $[11,8,12]$.

Durante o este do capítulo busca-se resolver problemas de minimização onde o conjunto viável é o fecho de um subconjunto aberto e convexo $S$ do $\mathbb{R}^{n}$. Para isto utilizar-se-ào as distâncias de Bregman:

DEFINIÇÃo 29. Seja $S \subset \mathbb{R}^{n}$ um convexo aberto, $h(\cdot)$ uma função real definida no fecho de $S$ e $D_{h}: \bar{S} \times S \mapsto \mathbb{R}$ definida por

$$
D_{h}(x, y)=h(x)-h(y)-\langle\nabla h(y), x-y\rangle \quad(>0 \text { para } x \neq y)
$$

$h(\cdot)$ é dita uma função de Bregman e $D_{h}(\cdot, \cdot)$ a distância de Bregman induzida por $h(\cdot)$ se valem

\footnotetext{
${ }^{1}$ Este capítulo está baseado na monografia Métodos de Ponto Proximal em Otimizaçào de Iusem [11].

${ }^{2}$ Bons textos de consulta sobre $\varphi$-divergencias são $[11,23,12,13]$. onde é possivel notar uma forte relação com distancias de Bregman.
} 
B1. (Diferenciabilidade) $h(\cdot)$ é continuamente diferenciável em $S$.

B2. (Conctridade tstrita) h( $\cdot$ ) é estritamente convexa e continua em $\bar{S}$.

B3. (Conjuntos de nível limitados) Para todo $a \in \mathbb{R}$ os conjuntos de nivel $\Gamma_{1}(y, a)=\left\{x \in \bar{S} \mid D_{h}(x, y) \leqq a\right\}$ e $\Gamma_{2}(x, a)=\{y \in S \mid$ $\left.D_{h}(x, y) \leqq a\right\}$ sào limitados para todo $y \in S$ e $x \in \bar{S}$.

B4. (Convergência da distância) Se $\left(y^{i}\right)_{i \in \mathbb{N}} \in S^{\prime}$ e $y^{i} \rightarrow y^{\infty} \in \bar{S}$ então $D_{h}\left(y^{\infty}, y^{i}\right) \rightarrow 0$.

B5. (Convergência de seqüência) Se $\left(x^{i}\right)_{i \in \mathbb{N}} \in \bar{S}$ e $\left(y^{i}\right)_{i \in \mathbb{N}} \in S$ são seqüências tais que $\left(x^{i}\right)_{i \in \mathbf{N}}$ é limitada, $y^{i} \rightarrow y^{\infty}$ e $D_{h}\left(x^{i}, y^{i}\right) \rightarrow 0$ então $x^{i} \rightarrow y^{\infty}$.

$\mathrm{O}$ conjunto 5 'é chamado de zona de $h(\cdot)$

Para efeito de referência as propriedades de funções de Bregman serão identificadas com os nomes entre parênteses colocados imediatamente antes de suas definições. Tais nomes não devem ser considerados descriçòes precisas das propriedades mas, sim, termos mnemônicos para facilitar a leitura do texto.

Para que este tipo de função seja usada como regularização no MPP, é necessário que ela guarde implicitamente a informação sobre o conjunto $\bar{S}$, que será identificado com o conjunto viável. Ver-se-á que isto é possível se $h(\cdot)$ obedecer também a condição: se vale

DEFINIÇÃO 30. a função de Bregman $h(\cdot)$ é coerciva na fronteira

B6. Se $\left(y^{i}\right)_{i \in \mathbf{N}} \in S$ e $y^{i} \rightarrow y^{\infty} \in \partial \bar{S}$ (fronteira de $\bar{S}$ ) entào para todo $x \in S$ vale

$$
\left\langle\nabla h\left(y^{i}\right), x-y^{i}\right\rangle \rightarrow-\infty
$$

Note que isto implica que $D_{h}\left(x, y^{i}\right) \rightarrow \infty$. pois $h(\cdot)$ é contínua em $\bar{S}$.

Basicamente esta propriedade diz que a distância de Bregman, entre um ponto da zona e uma seqüência que converge para a fronteira, tende a infinito, como se a seqüência estivesse divergindo.

Alguns exemplos de funçòes de Bregman com zona bastante simples, e com a propriedade de serem coercivas na fronteira, são:

- A zona $S=\mathbb{R}^{n}$.

Um exemplo de funçào de Bregman interessante é

$$
h(x)=\|x\|_{2}^{2},
$$


cuja distância de Bregman associada é:

$$
\begin{aligned}
D_{h}(x, y) & =\|x\|_{2}^{2}-\|y\|_{2}^{2}-\langle 2 y, x-y\rangle \\
& =\|x\|_{2}^{2}-2\langle y, x\rangle+\|y\|_{2}^{2} \\
& =\|x-y\|_{2}^{2} .
\end{aligned}
$$

Claramente estas funçòes obedecem a todas as condiçòes que caracterizam uma função de Bregman.

Este caso merece destaque, pois pode-se considerar que o método de pontos proximais obtido a partir de distâncias de Bregman (que será apresentado abaixo) estende o método de pontos proximais baseado na regularização Euclidiana.

- A zona $S$ é o interior de $\mathbb{R}_{+}^{n}$.

Neste caso, um exemplo de funçào de Bregman é $h(x)=$ $\sum_{i=1}^{n} x_{i} \ln x_{i}$, convencionando-se que $0 \ln 0=0$. A distância de Bregman associada é:

$$
\begin{aligned}
D_{h}(x, y) & =\sum_{i=1}^{n} x_{i} \ln x_{i}-\sum_{i=1}^{n} y_{i} \ln y_{i}-\sum_{i=1}^{n}\left(\left(\ln y_{i}+1\right)\left(x_{i}-y_{i}\right)\right) \\
& =\sum_{i=1}^{n}\left(x_{i} \ln x_{i}-x_{i} \ln y_{i}-x_{i}+y_{i}\right) \\
& =\sum_{i=1}^{n}\left(x_{i} \ln \frac{x_{i}}{y_{i}}-x_{i}+y_{i}\right) .
\end{aligned}
$$

A prova que estas funçòes obedecem as propriedade $B 1$ a $B 6$ é um pouco longa, e pouco elucidativa e, por isso, é apresentada no apêndice deste capítulo.

- A zona $S$ é o interior de uma caixa do $\mathbb{R}^{n}$, isto é:

$$
S=\left(a_{1}, b_{1}\right) \times \cdots \times\left(a_{n}, b_{n}\right),
$$

onde $a_{i}<b_{i}$ para $1 \leqq i \leqq n$.

Um exemplo de função de Bregman é:

$$
h(x)=\sum_{i=1}^{n}\left(\left(x_{i}-a_{i}\right) \ln \left(x_{i}-a_{i}\right)+\left(b_{i}-x_{i}\right) \ln \left(b_{i}-x_{i}\right)\right) .
$$

E a distância de Bregman associada fica:

$$
D_{h}(x, y)=\sum_{i=1}^{n}\left(\left(x_{i}-a_{i}\right) \ln \frac{x_{i}-a_{i}}{y_{i}-a_{i}}+\left(b_{i}-x_{i}\right) \ln \frac{b_{i}-x_{i}}{b_{i}-y_{i}}\right) \text {. }
$$


Mais uma vez a prova que estas funçòes obedecem as condiçòes de Bregman se encontra no apêndice.

Outros exemplos de funçòes de Bregman, inclusive para os casos da zona ser o interior de uma bola ou de um poliedro, podem ser encontrados no artigo [7].

Neste ponto, é conveniente provar que funçòes de Bregman obedecem a algumas propriedades que serão úteis para sua manipulação no restante do capítulo. Três resultados básicos estào reunidos abaixo. entào

Proposição 1. Se $h(\cdot)$ é uma funçào de Bregman com zona S

1. $D_{h}(x, y)=D_{h}(x, z)-D_{h}(z, y)=\langle\nabla h(y)-\nabla h(z) . z-x\rangle$, para todo $x \in \bar{S}$ e $y, z \in S$.

2. $\nabla_{x} D_{h}(x, y)=\nabla h(x)-\nabla h(y), \forall x, y \in S$.

3. $D_{h}(\cdot, y)$ é estritamente convexa para todo $y \in S$.

Prova. A prova está separada em três partes, correspondentes às partes da proposição.

1.

$$
\begin{gathered}
D_{h}(x, y)-D_{h}(x, z)-D_{h}(z, y)= \\
=h(x)-h(y)-\langle\nabla h(y), x-y\rangle-h(x)+h(z)+\langle\nabla h(z), x-z\rangle \\
\quad-h(z)+h(y)+\langle\nabla h(y), z-y\rangle \\
=\langle\nabla h(y), z-x\rangle-\langle\nabla h(z), z-x\rangle
\end{gathered}
$$

2. Trivial, basta aplicar regras àe diferenciação, lembrando que $y$ está fixo.

3. Segue imediatamente da convexidade estrita de $h(\cdot)$.

\section{O Método}

Apresenta-se. no contexto de programação convexa. um método de solução para problemas que obedecem a seguinte hipótese:

Hipótese 3. O conjunto viável $V$ é igual ao fecho de um conjunto convexo, aberto e não vazio, denotado por $S$. A função objetivo $f(\cdot)$, definida em $\bar{S}$ e com imagens reais, é convexa e contínua. Por fim $f(\cdot)$ 
é limitada inferiormente em $V=\bar{S}$. Um problema que obedece a estas condiçoes será chamado de PS:

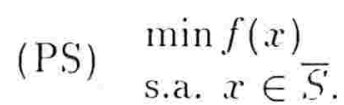

Neste caso. sempre que for possivel encontrar uma funçào de Bregman com zona Ś e coerciva na fronteira, pode-se utilizar uma variação do método de ponto proximal para a soluçào do problema. O método é particularmente interessante porque as minimizaçōes que sào feitas no passo iterativo podem ser resolvidas por algoritmos tradicionais de minimizaçào irrestrita, como feito em métodos de barreira [4]. A descriçào geral do método é dada por:

Método de Ponto Proximal com distância de Bregman (MPPB) Dada uma funsào de Bregman $h(\cdot)$, com zona $S$ e coerciva na fronteira:

1. Inicializaçio: Tome qualquer $x^{1} \in S$;

2. Iteraçào: Calcule $x^{i+1}$ por

$$
x^{i+1}=\underset{x \in \bar{S}}{\operatorname{argmin}}\left\{f_{i}(x)=f(x)+\lambda_{i} D_{h}\left(x, x^{i}\right)\right\} .
$$

onde $\left(\lambda_{i}\right)_{i \in \mathbf{N}}$ é uma seqüência de números reais positivos $\epsilon$ limitados superiormente por $\tilde{\lambda}$.

Note que a propriedade de coercividade faz com que a distância de Bregman desempenhe um papel semelhante ao de uma função barreira, impedindo que os iterados "saiam" do interior do conjunto viável. Este comentário é formalizado no lema abaixo:

LEMA 28. O MPPB está bem definido, isto $\dot{e}$, dado um ponto $x^{i} \in$ $S$, os iterados sào univocamente definidos e pertencem ao interior do conjunto viável, isto é, pertencem a S'.

Prova. Seja $/ \mathrm{um}$ limitante inferior de $f(\cdot)$ em $\bar{S}$. Segue imediatamente que a função minimizada à cada interação obedece à

$$
f_{i}(x) \geqq l+\lambda_{i} D_{h}\left(x, x^{i}\right)
$$

Pela condiçào de limitaçào dos conjuntos de nivel de funções de Bregman e pela continuidade de $D_{h}\left(\cdot, x^{i}\right)$, tem-se que os conjuntos de nível de $f_{i}(x)$ estào contidos em conjuntos fechados e limitados. Portanto, a cada iteraçào, a minimização irrestrita é equivalente a uma minimizaçào em um compacto. Por fim, $f_{i}(\cdot)$ é estritamente convexa, pois $f(\cdot)$ é convexa e a distância de Bregman é estritamente convexa na primeira variável (proposição 1). Deste modo, o mínimo calculado a cada iteraçào existe e é único. 
Assim, sabe-se que $x^{i+1}$ é o único ponto de $\bar{S}$ tal que

$$
\forall y \in \bar{S} . \quad f(y)+\lambda_{i} D_{h}\left(y, x^{i}\right) \geqq f\left(x^{i+1}\right)+\lambda_{i} D_{h}\left(x^{i+1} \cdot x^{i}\right) \Leftrightarrow
$$

$$
\begin{aligned}
\forall y \in \bar{S}, \quad f(y)+\lambda_{i}\left(h(y)-h\left(x^{i}\right)-\left\langle\nabla h\left(x^{i}\right), y-x^{i}\right\rangle\right) \geqq \\
f\left(x^{i+1}\right)+\lambda_{i}\left(h\left(x^{i+1}\right)-h\left(x^{i}\right)-\left\langle\nabla h\left(x^{i}\right), x^{i+1}-x^{i}\right\rangle\right) \Leftrightarrow
\end{aligned}
$$

$$
\begin{gathered}
\forall y \in \bar{S}, \quad f(y)+\lambda_{i} h(y) \geqq \\
f\left(x^{i+1}\right)+\lambda_{i} h\left(x^{i+1}\right)+\left\langle\lambda_{i} \nabla h\left(x^{i}\right), y-x^{i+1}\right\rangle \Leftrightarrow \\
\lambda_{i} \nabla h\left(x^{i}\right) \in \partial\left(f+\lambda_{i} h\right)\left(x^{i+1}\right)
\end{gathered}
$$

Deste modo, para concluir a prova basta mostrar que $\partial\left(f+\lambda_{i} h\right)(x)$ é vazio para todo ponto $x$ na fronteira de $S$.

Supondo, por contradição, que existe um ponto $x$ na fronteira de $S$ tal que $\left(f+\lambda_{i} h\right)(\cdot)$ possua um subgradiente $\gamma$ em $x$. Seja $z$ um elemento qualquer de $S$ e defina

$$
\alpha^{j}=\frac{1}{j+1} \quad \text { e } \quad y^{j}=\left(1-\alpha^{j}\right) x+\alpha^{j} z
$$

Usando estas definiçôes, tem-se que

$$
\forall j, y^{j} \in S \quad \text { e } \quad \lim _{j \rightarrow \infty} y^{j}=x \in \partial \bar{S}
$$

Pode-se concluir, então, que

$$
\begin{aligned}
& \alpha^{j}\langle\gamma, z-x\rangle=\left\langle\gamma, y^{j}-x\right\rangle \\
& \left.\leqq f\left(y^{j}\right)-f(x)+\lambda_{i}\left(h\left(y^{j}\right)-h(x)\right) \quad \text { [Def. } \gamma\right] \\
& \leqq f\left(y^{j}\right)-f(x)+\lambda_{i}\left\langle\nabla h\left(y^{j}\right), y^{j}-x\right\rangle \quad \text { [Convex. } h(\cdot) \text { ] } \\
& \left.\leqq \alpha^{j}(f(z)-f(x))+\lambda_{i}\left\langle\nabla h\left(y^{j}\right), y^{j}-x\right\rangle \quad \text { [C'onvex. } f(\cdot)\right] \\
& \left.=\alpha^{j}(f(z)-f(x))+\lambda_{i}\left\langle\nabla h\left(y^{j}\right), \frac{\alpha^{j}}{1-a^{j}}\left(z-y^{j}\right)\right\rangle \quad \text { [Def. } y^{j}\right]
\end{aligned}
$$

Portanto

$$
\frac{1-\alpha^{j}}{\lambda_{i}}(\langle\gamma, z-x\rangle+f(x)-f(z)) \leqq\left\langle\nabla h\left(y^{j}\right), z-y^{j}\right\rangle .
$$

Tomando o limite para $j \rightarrow \infty$ e lembrando da coercividade na fronteira de $h(\cdot)$ :

$$
\frac{1}{\lambda_{i}}(\langle\gamma, z-x\rangle+f(x)-f(z)) \leqq-\infty
$$


Lima contradiçào. Logo segue que. para todo $x \in \partial \bar{S}$.

$$
\partial\left(f+\lambda_{i} h\right)(x)=\emptyset .
$$

completando a prova que $x^{i+1}$ está em $S$.

Nota-se que aqui é possivel obter um candidato a separador ${ }^{3}$ :

$$
\begin{gathered}
\tilde{x}^{i}=\underset{x \in \bar{S}}{\operatorname{argmin}}\left\{f(x)+D_{h}\left(x, x^{i}\right)\right\} \Leftrightarrow \\
\exists \gamma_{f}^{i} \in \partial f\left(\tilde{x}^{i}\right): \gamma_{j}^{i}+\nabla h\left(\tilde{x}^{i}\right)-\nabla h\left(x^{i}\right)=0 \Rightarrow \\
f\left(x^{i}\right) \geqq f\left(\tilde{x}^{i}\right)+\left\langle\nabla h\left(x^{i}\right)-\nabla h\left(\tilde{x}^{i}\right), x^{i}-\tilde{x}^{i}\right\rangle .
\end{gathered}
$$

Na últimas expressòes foi usado o fato das seqüências de interesse estarem inteiramente contidas no aberto $S$ (lema anterior). Usando, então, a proposição 1 ítem $1, \operatorname{com} x=y=\widetilde{x}^{i} \mathrm{e} z=x^{i}$, obtém-se:

$$
f\left(x^{i}\right) \geqq f\left(\tilde{x}^{i}\right)+D_{h}\left(\tilde{x}^{i}, x^{i}\right)+D_{h}\left(x^{i}, \tilde{x}^{i}\right),
$$

sugerindo que $D_{h}\left(\widetilde{x}^{i}, x^{i}\right)+D_{i h}\left(x^{i}, \widetilde{x}^{i}\right)$ é um separador. Infelizmente não foi possível provar isio. A ciificuldade está no fato das propriedades exigidas sobre funçòes de Bregman não permitirem uma análise simples do que acontece com a diferença $D_{h}\left(\bar{x}, x^{i}\right)-D_{h}\left(\bar{x}, y^{i}\right)$, onde $\bar{x}$ é um ponto de $\bar{S}$, mesmo quando $\left(x^{i}\right)_{i \in \mathbb{N}}$ e $\left(y^{i}\right)_{i \in \mathbb{N}}$ possuem o mesmo limite. Assim, apesar de ser possivel afirmar que $\left(\widetilde{x}^{i}\right)_{i \in \mathbf{N}}$ e $\left(x^{i}\right)_{i \in \mathbf{N}}$ convergem para o mesmo ponto, nào foi possível provar que a seqüência gerada converge para uma solução.

2.1. Prova de convergência do MPPB. Uma vez que se sabe que o método está bem definido, busca-se agora provar a convergência do MPPB.

Inicialmente. mostra-se que o método possui uma propriedade semelhante a Féjer monotonicidade. Este resultado será usado na demonstraçào do teorema de convergência.

LEMA 29. Seja $x^{*}$ uma solução qualquer do PS. A seqüencia gerada pelo $M P P B$ tem a propriedade

$$
D_{h}\left(x^{*}, x^{i+1}\right) \leqq D_{h}\left(x^{*}, x^{i}\right)-D_{h}\left(x^{i+1}, x^{i}\right) .
$$

Prova. Usando a proposição 1 , item $1, \operatorname{com} x=x^{\times}, y=x^{i} \mathrm{e}$ $z=x^{i+1}$. tem-se

$$
\begin{array}{r}
D_{h}\left(x^{*}, x^{i}\right)-D_{h}\left(x^{*}, x^{i+1}\right)-D_{h}\left(x^{i+1}, x^{i}\right)= \\
\left\langle\nabla h\left(x^{i}\right)-\nabla h\left(x^{i+1}\right), x^{i+1}-x^{*}\right\rangle .
\end{array}
$$

\footnotetext{
${ }^{3}$ Por simplicidade considerou-se que a seqüencia de pesos $\lambda_{i}$ vale sempre 1 .
} 
Da definiçào de $x^{i+1}$ e como, pelo lema anterior. $x^{i+1} \in S$ que é aberto

$$
\begin{gathered}
0 \in \partial\left(f+\lambda_{i} D_{h}\left(\cdot, x^{i}\right)\right)\left(x^{i+1}\right) \quad[\text { Corolário } 8] \Leftrightarrow \\
\exists \gamma_{j} \in \partial f\left(x^{i+1}\right): \gamma_{f}+\lambda_{i}\left(\Gamma h\left(x^{i+1}\right)-\nabla h\left(x^{i}\right)\right)=0 \Leftrightarrow \\
\gamma_{f}=\lambda_{i}\left(\nabla h\left(x^{i}\right)-\nabla h\left(x^{i+1}\right)\right) \in \partial f\left(x^{i+1}\right) .
\end{gathered}
$$

A seg'ında linha é conseqüência da proposiçào 1 e da regra da soma para subgradientes, no caso de uma das funçòes diferenciáveis (teorema 15).

De (21) conclui-se

$$
\begin{aligned}
D_{h}\left(x^{*} \cdot x^{i}\right)-D_{h}\left(x^{*}, x^{i+1}\right)-D_{h}\left(x^{i+1}, x^{i}\right) & =\frac{1}{\lambda_{i}}\left\langle\partial j, x^{i+1}-x^{*}\right\rangle \\
& \geqq \frac{1}{\lambda_{i}}\left(f\left(x^{i+1}\right)-f\left(x^{*}\right)\right) \\
& \geqq 0
\end{aligned}
$$

onde a primeira desigualdade é conseqüência da definição de subgradiente e a segunda do fato de $x^{*}$ ser uma soluçào da minimizaçào de $f(\cdot)$ em $\bar{S}$.

Mais uma vez é importante destacar o fato desta propriedade é análoga a Fejér monotonicidade, porém usando a "métrica" $D_{h}(\cdot, \cdot)$.

Um corolário importante é:

Corolário 9. Seja $x^{*}$ uma solução qualquer do PS e $\left(x^{i}\right)_{i \in \mathbb{N}}$ uma seqüência gerada pelo MPPB. Então a seqüência $D_{h}\left(x^{*}, x^{i}\right)$ converge para algum valor real.

Prova. Do lema anterior, conclui-se que a sequiência $D_{h}\left(x^{*}, x^{i}\right)$ é não crescente e, como é limitada inferiormente por 0 , a seqüência é convergente.

Por fim. já é possível enunciar e provar o teorema de convergência do método.

TEOrena 26. Se o problema PS tem soluçâo entào a seqüência $\left(x^{i}\right)_{i \in \mathbf{N}}$ gerada pelo Método de Ponto Proximal com distâncias de Bregman converge a uma soluçâo.

Prova. A prova será dividida em três partes.

1. A seqüência é limitada e, se uma subseqüência $x^{j_{t}}$ é convergente a um ponto $\bar{x}$ então a seqüência $x^{j_{t}+1}$ também converge para $\bar{x}$.

Seja $x^{*}$ uma solução qualquer do PS, que existe por hipótese. O lema 29 implica que a seqüência $D_{h}\left(x^{*} . x^{i}\right)$ é não crescente, portanto $D_{h}\left(x^{*}, x^{i}\right) \leqq D_{h}\left(x^{*}, x^{1}\right)$ e a propriedade de conjuntos de nível limitados das funçòes de Bregman diz que $\left(x^{i}\right)_{i \in \mathbf{N}}$ é limitada. 
Mais ainda. lembrando que o corolário 9 afirma que $D_{h}\left(. r^{*} . x^{i}\right)$ é convergente. temos. pelo lema 29 :

$$
\begin{gathered}
0 \leqq D_{h}\left(x^{i+1}, x^{i}\right) \leqq \underbrace{D_{h}\left(x^{\times} \cdot x^{i}\right)-D_{h}\left(x^{\times} \cdot x^{i+1}\right)}_{\rightarrow 0} \Rightarrow \\
D_{h}\left(x^{i+1}, x^{i}\right) \rightarrow 0 .
\end{gathered}
$$

Portanto, qualquer que seja a subseqüência convergente $x^{j_{t}}$ com limite $\bar{x}$. da expressão anterior e da propriedade de funções de Bregman chamada de convergência de seqüências. conclui-se que $i^{i_{1}+1} \rightarrow \bar{x}$.

2. Todos os pontos de acumulação de $\left(x^{i}\right)_{i \in \mathbb{N}}$ são soluções do PS.

Seja $x^{*}$ uma solução do PS, $\tilde{\lambda}$ um limitante superior da seqüência $\left(\lambda^{i}\right)_{i \in \mathbf{N}}$ (que existe por hipótese na definição do método) e $x^{j_{t}} \rightarrow \bar{x}$ uma subseqüência convergente.

Reescrevendo a equaçào 22 , da demonstraçào do lema 29

$$
\begin{aligned}
D_{h}\left(x^{*}, x^{j_{l}}\right)-D_{h}\left(x^{*}, x^{j_{1}+1}\right)-D_{h}\left(x^{j_{t}+1}, x^{j_{i}}\right) & \geqq \frac{1}{\lambda_{i}}\left(f\left(x^{j_{1}+1}\right)-f\left(x^{*}\right)\right) \\
& \geqq \frac{1}{\tilde{\lambda}}\left(f\left(x^{j_{i}+1}\right)-f\left(x^{*}\right)\right) \\
& \geqq 0 .
\end{aligned}
$$

A primeira expressào converge para 0 , pois $D_{h}\left(x^{j_{t}+1} \cdot x^{j_{t}}\right) \rightarrow 0$ por (23) e $D\left(x^{*}, x^{i}\right)$ converge (corolário 9).

Segue que

$$
\lim _{i \rightarrow \infty} f\left(x^{j_{1}+1}\right)=f\left(x^{*}\right) .
$$

E assim, da continuidade de $f(\cdot)$ e do fato de $\lim _{i \rightarrow \infty} f\left(x^{j_{t}}\right)=$ $\lim _{i \rightarrow \infty} f\left(x^{j_{1}+1}\right)$, provado no item anterior, conclui-se que

$$
f(\bar{x})=f\left(x^{*}\right) .
$$

Por fim, como $\left(x^{i}\right)_{i \in \mathbf{N}} \in S$, tem-se que $\bar{x} \in \bar{S}$. Portanto $\bar{x}$ é viável e, lembrando de (24), uma soluçào.

3. $\left(x^{i}\right)_{i \in \mathbb{N}}$ possui um único ponto de acumulaçào.

Seja $\bar{x}$ um ponto de acumulação, que existe pelo ítem 1 , e $x^{j_{t}}$ uma subseqüência convergente a $\bar{x}$. 
Sabe-se que

$$
\begin{array}{lr}
D_{h}\left(\bar{x} . r^{\prime \prime}\right) \rightarrow 0 & \text { [Pela Prop. C'onv. de Distâncias] } \\
\bar{x} \text { resolve o PS } & \text { [Pelo ítem anterior] }
\end{array}
$$

Como $D_{h}\left(\bar{x}, x^{i}\right)$ é não crescente com uma subseqüência convergindo para 0 (como está destacado acima), a seqüência converge para 0 .

Seja $x^{k_{t}} \rightarrow \tilde{x}$ uma subseqüência convergente qualquer.

Defina as seqüências

$$
\begin{aligned}
y^{i} & =\bar{x} & \in \bar{S} \\
z^{i}=x^{k_{t}} & \rightarrow \tilde{x} & \in S
\end{aligned}
$$

Segue imediatamente que $y^{i}$ é limitada e que o limite de $D_{h}\left(y^{i}, z^{i}\right)$ é 0 . A propriedade de convergência de seqüências garante que

$$
\vec{x}=\lim _{i \rightarrow \infty} y^{i}=\lim _{i \rightarrow \infty} z^{i}=\tilde{x}
$$

O teorema segue da junção dos três últimos ítens.

Existem outros resultados sobre métodos proximais baseados no uso de distâncias de Bregman que não serão tratados nesta dissertação. Por isto, apresenta-se uma pequena bibliografia que pode ser utilizada como ponto de partida para pesquisa. Textos gerais são $[11,8]$. Em $[11]$ faz-se a análise da taxa de convergência destes algoritmos. Em $[6,11]$ estuda-se sua utilidade para a solução de problemas de desigualdade variacional. Im exemplo do uso de funçòes de Bregman em algoritmos de pontos interiores diferentes do MPP é [7].

\section{Apêndice}

Neste apêndice demonstra-se que os exemplos de funções de Bregman que foram apresentados na seção 1 obedecem às condições da definição ( $B 1$ a $B 6)$.

3.1. A zona é o interior do ortante positivo $\left(\mathbb{R}_{++}^{n}\right)$. O exemplo de função de Bregman apresentado foi:

$$
h(x)=\sum_{i=1}^{n} x_{i} \ln x_{i}, \text { onde } 0 \ln 0=0 .
$$


E a respectiva distância de Bregman é dada por:

$$
D_{h}(x, y)=\sum_{i=1}^{n}\left(x_{i} \ln \frac{x_{i}}{y_{i}}-x_{i}+y_{i}\right) .
$$

Note que a estrutura de separabilidade presente nestas funções, permitirá que manipulaçòes feitas apenas nos reais sejam facilmente generalizadas para o $\mathbb{R}^{n}$.

B1. $h(\cdot)$ é continuamente diferenciável em $S$.

Prova. Trivial.

B2. $h(\cdot)$ é estritamente convexa e contínua em $\bar{S}$.

Prova. Chamemos de $f(\cdot)$ a restrição de $h(\cdot)$ a uma dimensão. Isto é $f(x)=x \ln x$. Prova-se primeiro que $f(\cdot)$ é estritamente convexa em $\mathbb{R}_{+}$.

Calcula-se:

$$
\begin{aligned}
f^{\prime}(x) & =\ln x+1, \\
f^{\prime \prime}(x) & =\frac{1}{x}>0, \text { se } x>0 .
\end{aligned}
$$

Logo segue a estrita convexidade de $f(\cdot)$ em $\mathbb{R}_{++}$. Resta provar que $f(\cdot)$ é estritamente convexa no 0 . Considere a um ponto qualquer de $\mathbb{R}_{++}$e $\lambda \in(0,1)$, tem-se

$$
\begin{array}{rlr}
f((1-\lambda) 0+\lambda a) & =\lambda a \ln \lambda a & \\
& <\lambda a \ln a \quad[\ln (\cdot) \text { é crescente }] \\
& =(1-\lambda) f(0)+\lambda f(a) .
\end{array}
$$

Assim, tomando $a$ e $b$ pontos distintos do $\mathbb{R}_{+}^{n}$ e $\lambda \in(0,1)$ tem-se

$$
\begin{aligned}
h((1-\lambda) a+\lambda b) & =\sum_{i=1}^{n}\left((1-\lambda) a_{i}+\lambda b_{i}\right) \ln \left((1-\lambda) a_{i}+\lambda b_{i}\right) \\
& <\sum_{i=1}^{n}\left((1-\lambda) a_{i} \ln a_{i}+\lambda b_{i} \ln b_{i}\right) \\
& =(1-\lambda) h(a)+\lambda h(b) .
\end{aligned}
$$

Agora prova-se a continuidade em $\mathbb{R}_{+}^{n}$. Observe que é suficiente provar a continuidade de $f(\cdot)$, que é claramente contínua 
em $\mathbb{R}_{++}$. Toma-se

$$
\begin{aligned}
\lim _{x \rightarrow 0^{+}} x \ln x & =-\lim _{x \rightarrow 0^{+}}-x \ln x \\
& =-\lim _{x \rightarrow 0^{+}} \frac{1 / x}{-1 / x^{2}} \quad \text { [L'Hôpital] } \\
& =0 .
\end{aligned}
$$

Portanto a convenção $0 \ln 0=0$ garante a continuidade.

B3. Para todo $\alpha \in \mathbb{R}$ os conjuntos de nível $\Gamma_{1}(y, \alpha)=\{x \in \bar{S} \mid$ $\left.D_{h}(x, y) \leqq \alpha\right\}$ e $\Gamma_{2}(x, \alpha)=\left\{y \in S \mid D_{h}(x, y) \leqq \alpha\right\}$ sào limitados para todo $y \in S$ e $x \in \bar{S}$.

Prova. Considere primeiro o conjunto de nível $\Gamma_{1}(y, \alpha)=$ $\left\{x \in \mathbb{R}_{+} \mid D_{h}(x, y) \leqq \alpha\right\}$, com $y \in \mathbb{R}_{++}$. Mais uma vez, basta considerar o caso unidimensional, limitando assim cada componente. Como $x$ é positivo, falta apenas obter um limitante superior.

$$
\begin{aligned}
D_{h}(x, y) & \leqq \alpha \Leftrightarrow \\
x \ln \frac{x}{y}-x+y & \leqq \alpha \Leftrightarrow \\
x \ln x-x \ln y-x+y & \leqq \alpha \Leftrightarrow \\
x(\ln x-\ln y-1) & \leqq \alpha-y
\end{aligned}
$$

Supõe-se, por contradição que $x$ não é limitado superiormente. Tomando o limite, para $x \rightarrow \infty$, na expressão 25 , tem-se uma contradição.

Resta analisar o conjunto de nível $\Gamma_{2}(x, \alpha)=\left\{y \in \mathbb{R}_{++} \mid\right.$ $\left.D_{h}(x, y) \leqq \alpha\right\}$, onde $x \in \mathbb{R}_{+}$. Como acima, pode-se considerar apenas o caso unidimensional e buscar a limitação superior.

$$
\begin{aligned}
D_{h}(x, y) & \leqq \alpha \Leftrightarrow \\
x \ln \frac{x}{y}-x+y & \leqq \alpha \Leftrightarrow \\
y-x \ln y & \leqq \alpha-x \ln x+x
\end{aligned}
$$

E mais uma vez a técnica de tomar o limite na expressào anterior garante que $y$ é limitado.

B4. Se $\left(y^{i}\right)_{i \in \mathbf{N}} \in S^{\prime}$ e $y^{i} \rightarrow y^{\infty} \in \overline{S^{i}}$ então $D_{h}\left(y^{\infty}, y^{i}\right) \rightarrow 0$

Prova. Basta provar que cada termo de $D_{h}\left(y^{\infty}, y^{i}\right)$ converge para 0 , o que é equivalente a considerar o caso unidimensional. Assim, existem duas possibilidades. 
- $y^{x}=0$. Neste caso

$$
D_{h}\left(y^{\infty} \cdot y^{i}\right)=0 \ln \frac{0}{y^{i}}-0+y^{i}=y^{i} \rightarrow 0 .
$$

- $y^{x}>0$. o que implica

$$
\begin{aligned}
D_{h}\left(y^{\infty}, y^{i}\right) & =y^{\infty} \ln \frac{y^{\infty}}{y^{i}}-y^{\infty}+y^{i} \\
& \rightarrow y^{\infty} \ln 1-y^{\infty}+y^{\infty} \quad \text { [Por continuidade] } \\
& =0 .
\end{aligned}
$$

B.5. Se $\left(x^{i}\right)_{i \in \mathbf{N}} \in \bar{S}$ e $\left(y^{i}\right)_{i \in \mathbf{N}} \in S$ são seqüências tais que $\left(x^{i}\right)_{i \in \mathbf{N}}$ é limitada, $y^{i} \rightarrow y^{\infty}$ e $D_{h}\left(x^{i}, y^{i}\right) \rightarrow 0$ então $x^{i} \rightarrow y^{\infty}$.

Prova. Primeiro observe que, como a distância de Bregman é soma de termos positivos, cada termo da soma converge para zero. Portanto é possível tratar a convergência de cada componente isolada. O que, mais uma vez, equivale a tratar o caso unidimensional. Tome uma subseqüência convergente de $\left(x^{i}\right)_{i \in \mathbf{N}}$ a um ponto $x^{\infty}$. Deve-se provar que $x^{\infty}=y^{\infty}$. Sem perda de generalidade, considera-se que a seqüência converge. Lembrando que

$$
D_{h}\left(x^{i}, y^{i}\right)=x^{i} \ln \frac{x^{i}}{y^{i}}-x^{i}+y^{i},
$$

Há dois casos para analisar:

- $y^{\infty} \neq 0$. Tomando o limite na expressào 26 , tem-se

$$
\begin{aligned}
0 & =x^{\infty} \ln \frac{x^{\infty}}{y^{\infty}}-x^{\infty}+y^{\infty} \\
& =x^{\infty} \ln x^{\infty}-y^{\infty} \ln y^{\infty}-\left(\ln y^{\infty}+1\right)\left(x^{\infty}-y^{\infty}\right) .
\end{aligned}
$$

A última expressào implica que $x^{\infty}=y^{\infty}$, pois $x \ln x$ é estritamente convexa em $\mathbb{R}_{+}$e diferenciável em $y^{\infty} \neq 0$.

- $y^{\infty}=0$. Supondo, por contradição, que $x^{\infty} \neq 0$. então

$$
\frac{x^{i}}{y^{i}} \rightarrow \infty
$$

Uma vez que $x^{i} \rightarrow x^{\infty}>0$ e $y^{i} \rightarrow 0$. Isto, pela equação 26 , contraria a convergência de $D_{h}\left(x^{i}, y^{i}\right)$ a 0 .

B6. Se $\left(y^{i}\right)_{i \in \mathbf{N}} \in S$ e $y^{i} \rightarrow y^{\infty} \in \partial S$ então para todo $x \in S$ vale

$$
\left\langle\nabla h\left(y^{i}\right), x-y^{i}\right\rangle \rightarrow-\infty \text {. }
$$


Prova. Lembrando que uma seqüência $\left(y^{i}\right)_{i \in \mathbf{N}}$ de pontos se aproxima da fronteira de $\mathbb{R}_{++}^{\prime \prime}$ se pelo menos uma coordenada se aproxima de zero. pode-se notar que. para

$$
\left\langle\nabla h\left(y^{i}\right), x-y^{i}\right\rangle \rightarrow-\infty .
$$

basta que isto ocorra para cada coordenada que se aproxima de 0 . Deste modo pode-se considerar o caso unidimensional. Tem-se

$$
\begin{aligned}
& y^{i} \rightarrow 0 \quad \text { e } \quad x>0 \Rightarrow \\
& \left(\ln y^{i}-1\right)\left(x-y^{i}\right) \rightarrow-\infty \text {. }
\end{aligned}
$$

Completando a prova de que a função proposta é realmente uma função de Bregman coerciva na fronteira de $\mathbb{R}_{++}$.

\subsection{A zona é o interior de uma caixa.}

$$
\bar{S}=\left(a_{i}, b_{i}\right) \times \cdots \times\left(a_{n}, b_{n}\right),
$$

onde $a_{i}<b_{i}$ para $1 \leqq i \leqq n$.

O exemplo de funçào de Bregman introduzido foi:

$$
h(x)=\sum_{i=1}^{n}\left(\left(x_{i}-a_{i}\right) \ln \left(x_{i}-a_{i}\right)+\left(b_{i}-x_{i}\right) \ln \left(b_{i}-x_{i}\right)\right) .
$$

Cuja distância de Bregman associada é:

$$
D_{h}(x, y)=\sum_{i=1}^{n}\left(\left(x_{i}-a_{i}\right) \ln \frac{x_{i}-a_{i}}{y_{i}-a_{i}}+\left(b_{i}-x_{i}\right) \ln \frac{b_{i}-x_{i}}{b_{i}-y_{i}}\right) .
$$

Note a semelhança entre este caso e o anterior (ortante positivo). Mais uma vez as funçòes envolvidas possuem importante estrutura de separabilidade, permitindo que as manipulações nos reais sejam facilmente estendidas. Também fica claro que as provas de validade das propriedades de funções de Bregman são análogas às provas da subseção 3.1. Por isso apresentar-se-á apenas um esboço das demonstrações, correlacionando-as com as anteriores e apontando as diferenças existentes.

B1. $h(\cdot)$ é continuamente diferenciável em $S$.

Prova. Trivial.

B2. $h(\cdot)$ é estritamente convexa e contínua em $\overline{S^{\prime}}$. 
Prova. A prova é parecida com o exemplo anterior. Porém. aqui deve-se mostrar separadamente a convexidade estrita de duas funções: $(x-a) \ln (x-a)$ em $[a, x)$ e $(b-x) \ln (b-x)$ em $(-\infty . b]$. Segue convexidade estrita de $h(\cdot)$ na intersecção dos intervalos, ou seja $[a, b]$.

A prova de continuidade é basicamente a mesma.

B3. Os conjuntos de nível são limitados.

Esta é a única prova realmente mais simples, se lembrarmos que a zona considerada é limitada. Como os conjuntos de nível estão contidos na zona o resultado é trivial.

B4. Se $\left(y^{i}\right)_{i \in \mathbf{N}} \in S$ e $y^{i} \rightarrow y^{\infty} \in \bar{S}$ entào $D_{h}\left(y^{\infty}, y^{i}\right) \rightarrow 0$

Prova. Totalmente análoga ao mesmo ítem na subseção anterior.

B5. Se $\left(x^{i}\right)_{i \in \mathbf{N}} \in \bar{S}$ e $\left(y^{i}\right)_{i \in \mathbf{N}} \in S$ são seqüências tais que $\left(x^{i}\right)_{i \in \mathbf{N}}$ é limitada, $y^{i} \rightarrow y^{\infty}$ e $D_{h}\left(x^{i}, y^{i}\right) \rightarrow 0$ entào $x^{i} \rightarrow y^{\infty}$.

Prova. Semelhante à prova anterior, com o cuidado de se considerar a função $(x-a) \ln ((x-a) /(y-a))$, para o caso de $y^{\infty}=a$ e a função $(b-x) \ln ((b-x) /(b-a))$ quando $y^{\infty}=b$.

B6. $h(\cdot)$ é coerciva na fronteira.

Prova. Semelhante à prova da subseçào 3.1, tomando o cuidado de considerar, como no ítem anterior, a função correta para os casos de $y^{i} \rightarrow a$ ou $b$. 


\section{('APíTULO 6}

\section{Conclusão}

Esta dissertação é orientada para o estudo de métodos de ponto proximal. apresentando duas de suas formas mais importantes: com regularizaçào Euclidiana e com regularizaçòes baseadas em distâncias de Bregman. Além disto, procurando fazer o texto razoavelmente autocontido, foi feita uma revisào de análise convexa.

O ponto fundamental, que deve ser enfatizado, é o uso de separadores como possível abordagem para o estudo do MPP. Este enfoque mostrou ser válido ao permitir uma generalizaçào do MPP com regularização Euclidiana.

A partir desta contribuição aos métodos de ponto proximal, abre-se naturalmente algumas linhas de pesquisa:

1. Extensão dos resultados, seguindo o mesmo enfoque, para funções que não envolvam normas, em particular, para funções de Bregman e $\varphi$-divergências[11].

2. Busca de novos métodos de Lagrangianos baseados nos métodos de ponto proximal propostos.

3. Extensão dos resultados para operadores monótonos e solução de problemas de desigualdade variacional. 


\section{Referências Bibliográficas}

[1] M. Avriel. Nonlinear Programming: Analysis and Methods. Prentice-Hall, 1976.

[2] C. Berge. Espaces Topologiques. Dunod, 2 edition, 1966.

[3] D. Bertsekas. Constrained Optimization and Lagrange Multipliers. Academic Press, New York, 1982.

[4] D. Bertsekas. Nonlinear Programming. Athena Scientific, 1995.

[5] L Bregman. The relaxation method of finding the common points of convex sets and its application to the solution of problems in convex programming. USSR Computational Mathematics and Mathematical Physics, 7:200-217, 1967.

[6] R. S. Burachik. Generalized Proximal Point Methods for the Variational Inequality Problem. P!ıD thesis, Instituto de Matemática Pura e Aplicada. Impa, 1995.

[7] Y. Censor, A. Iusem, and S.. Zenios. An interior point method with Bregman functions for the variational inequality problem with paramonotone operators. Preprint, 1996. Será Publicado na Mathematical Programming.

[8] Y. Censor and J Zenios. The proximal minimization algorithms with Dfunctions. Journal of Optimization: Theory and Applications, 73:451-464, 1992.

[9] B. C. Eaves and W. I. Zangwill. Generalized cutting plane algorithms. SIAM Journal on Control, 9:529-542, novembro 1971.

[10] A. F. P. de C. Humes and C. Humes. Programação Linear: Um Primeiro Curso. SBMAC, 1986. Livro de minicurso do IX Congresso Nacional de Matemática Aplicada e Computacional.

[11] A. Iusem. Métodos de Ponto Proximal em Otimização. Instituto de Matemática Pura e Aplicada - CNPq, 1995. Livro do $20^{\circ}$ Colóquio Brasileiro de Matemática.

[12] A. Iusem and M. Teboulle. On the convergence rate of entropic proximal minimization algorithms. Computational and Applied Mathematics, 12:153-168, 1993.

[13] A. Iusem, M. Teboulle, and B. Svaiter. Entropy-like proximal methods in covex programming. Mathematics of Operations Research, 19(4):790-814. Novembro 1994.

[14] O. L. Mangasarian. Nonlinear Programming. McGraw-Hill Book Company, New York, 1969.

[15] George J. Minty. Monotone (nonlinear) operators in Hilbert spaces. Duke Mathematical Journal, 29:341-346, 1962.

[16] George J Minty. On the monotonicity of the gradiente of a convex function. Pacific Journal of Mathematics, 14:243-247, 1964.

[17] J. Moreau. Proximité et dualité dans un espace hilbertien. Bull. Soc. Math. France, 93:273-299, 1965. 
[18] R. T. Rockafellar. Convex Analysis. Princeton University Press, 1970.

[19] R. T. Rockafellar. Augmented lagrangians and applications of the proximal point algorithm in convex programming. Mathematics of Operations Research, $1: 97-116,1976$

[20] R. T Rockafellar. Monotone operators and the proximal point algorithm. SIAM Journal on Control and Optimization, 14:887-898, Agosto 1976.

[21] R. T. Rockafellar. On the maximal monotonicity of subdifferential mappings. Pacific Journal of Mathematics, 33:200-216, 1976.

[22] R. T. Rockafellar. The Theory of Subgradients and its Applications to Problems of Optimization: Convex e Nonconvex Functions. Heldermann Verlang Berlin, 1979 .

[23] M. Teboulle. Entropic proximal methods with aplications to nonlinear programming. Mathematics of Operations Research, 17:670-690, 1992.

[24] W. I. Zangwill. Nonlinear Programming: An Unified Aproach. Prentice Hall, Englewood Cliffs, 1969. 